\title{
Affektive Epistemologien des Feldes
}

\subsection{Modelle affektiver Affenbegegnung im Reisebericht des 19. Jahrhunderts}

\subsubsection{Reisen zu den Affen}

Bevor mit Sherwood Washburn und Louis Leakey zwei Leitfiguren der Anthropologie in den 1950er bzw. 1960er Jahren auf die Idee kamen, junge Forscher/ innen ,ins Feld' zu schicken, wurde Wissen über Tiere überwiegend aus der Beobachtung gefangener oder der Autopsie toter Exemplare generiert. Das Problem, mithilfe dieser entweder teil-domestizierten oder ,traumatisierten ' Zoo- und Forschungstiere oder anhand des toten Körpers valide Erkenntnisse über das Leben und Verhalten der Art zu erhalten, lag auf der Hand und rückte im Rahmen von wissenschaftshistorischen Verschiebungen in den Vordergrund: ${ }^{1}$ Die Beobachtung nach Europa verbrachter gefangener Affen und die anatomische Forschung am Skelett, wie sie auch Edward Tysons Orang-outang zugrunde lag, wurde zwar in der Moderne durch systematische Tierversuche im Labor ergänzt, die das Bedürfnis spiegeln, das Forscher/innen-Subjekt als Wahrnehmungsinstanz $\mathrm{zu}$ neutralisieren und das Untersuchungsobjekt so stabil und kontrollierbar $\mathrm{zu}$ halten, wie es die epistemische Verlagerung zur wissenschaftlichen Objektivität gebietet. Beides fördert - in Texten - Ergebnisse zur Physis der Art und bestimmten manipulierbaren Formen des Verhaltens zutage, kann aber kaum valide Aussagen über das Verhalten von Tieren in ihrer, natürlichen“ Umgebung liefern.

Es gibt jedoch eine lange Tradition der Erkundung der ,natürlichen “ im Gegensatz zur ,zivilisierten' Welt, an die die primatologischen Feldforschungsreisen anknüpfen konnten: die Entdeckungs- und Forschungsreisen, Explorations, Travels, und Adventures, in deren Beschreibungen sich ca. ab Ende des 18.

\footnotetext{
${ }^{1}$ Vgl. Strum/Fedigan: Changing Views of Primate Society, S. 6 ff.
} 
Jahrhunderts in einer Vielzahl von publikumswirksamen Publikationen zunehmend bereits anekdotisch proto-ethologische Beobachtungen von Tieren finden lassen. ${ }^{2}$ Emotionalität im Zusammenhang mit der Erkundung anderer Kulturen, Landstriche und einer als unberührt imaginierten ,exotischen' Natur ist bereits Gegenstand vielzähliger Forschungsarbeiten. ${ }^{3}$ Für die vorliegende Arbeit ist grundsätzlich dreierlei von Interesse: Erstens erzeugen diese Reiseberichte von Naturforscher/ innen und sogenannten Abenteurer/innen ${ }^{4}$ die Forschungsreise und den oder die Forschungsreisende/n als Gegenstand von Literatur, sei dies in der Form des seriellen Zeitungsberichts (so z. B. Henry Morton Stanleys ,Dispatches' an den New York Herald und den Daily Telegraph), ${ }^{5}$ als Einzelpublikation oder aber als

\footnotetext{
${ }^{2}$ Eine strenge Unterscheidung zwischen Reise- und Forschungsbericht soll im Kontext dieser Arbeit nicht forciert werden. Wie Peter J. Brenner in seinem Forschungsüberblick bereits ausführlich dargestellt hat, ist der Reisebericht als „erzählende Darstellung einer realen Reise“ und „Vermittlung authentischer, durch Autopsie gewonnener Informationen“ als Gattung nicht durch nominalistische Setzungen bestimmt, sondern stellt wesentlich ein soziales Phänomen dar, dessen Funktion und Form von gesellschaftlichen Erwartungen abhängt. Ähnliches darf auch für den Forschungsbericht gelten. In seiner idealen Form bringt den Reise- als Forschungsbericht das späte 18. Jahrhundert hervor. Im Gegensatz zu früheren Reise- und Entdeckungstexten dient der Reisebericht fortan und vor allem ab Mitte des 19. Jahrhunderts als Medium, in dem sich weniger Welt- als Ich-Erfahrungen durch die verarbeitende Aneignung des Fremden artikulieren lassen. Brenner, Peter J.: Der Reisebericht in der deutschen Literatur. Tübingen 1990, S. 1, 159 ff. Siehe zudem Barton, Geoff (Hg.): Travel Writing. Oxford 1993; Korte, Barbara: Der englische Reisebericht. Von der Pilgerfahrt bis zur Postmoderne. Darmstadt 1996; Young, Tim: Travellers in Africa. British Travelogues, 1850-1900. Manchester/New York 1994. Zur Literarizität des Forschungsreiseberichts siehe Görbert, Johannes: Die Vertextung der Welt. Forschungsreisen als Literatur bei Georg Forster, Alexander von Humboldt und Adelbert von Chamisso. Berlin 2014.
}

${ }^{3}$ Vgl. z. B Johannes Fabians Dekonstruktion des rationalen, selbstbewussten Subjekts des Afrikareiseberichts im 19. Jahrhundert anhand seines emotionalen Erlebens im erotischen Kontext, Brenners Zuspitzung des empfindsamen Reiseberichts des ausgehenden 18. Jahrhunderts und Porters psychoanalytische Lesart des Begehrens der Reisenden: Fabian, Johannes: Out of Our Minds: Reason and Madness in the Exploration of Central Africa. Berkeley 2000, S. 78-101; Brenner: Der Reisebericht, S. 190 ff.; Porter, Dennis: Haunted Journeys. Desire and Transgression in European Travel Writing. Princeton 1991. Duncan/Gregory schließlich beschreiben die grundsätzlich emotionale Färbung der Reise in der Moderne als ,,more than a necessary evil [...], but rather [...] as an end in itself, as a form of pure pleasure“. Duncan, James/Gregory, Derek: Introduction. In: Dies. (Hg.): Writes of Passage: Reading Travel Writing. London/New York 1999, S. 6.

${ }^{4}$ Die Designation ,Abenteurer“ scheint überwiegend männlichen Reisenden verliehen zu werden. Siehe aber die sich stetig mehrende Forschungsliteratur zu weiblichen Reisenden auch im 18. und 19. Jahrhundert, bspw. Griep, Wolfgang/Pelz, Annegret: Frauen reisen. Ein bibliographisches Verzeichnis deutschsprachiger Frauenreisen 1700 bis 1810. Bremen 1995; Stamm, Ulrike: Der Orient der Frauen. Reiseberichte deutschsprachiger Autorinnen im frühen 19. Jahrhundert. Köln u. a. 2010; Blunt, Alison: Travel, Gender, and Imperialism. Mary Kingsley and West Africa, New York u. a. 1994; Bijon, Béatrice/Gacon, Gérard (Hg.): In-between Two Worlds. Narratives by Female Explorers and Travellers, 1850-1945. New York 2009.

${ }^{5}$ Siehe Bennett, Norman R. (Hg.): Stanley's Despatches to the NEW YORK HERALD 1871-1872, 1874-1877. Boston 1970; Daerden, Peter/Wynants, Maurits: Inventory of the Henry M. Stanley Archives. Tervuren 2005, S. 264 sowie bspw. Stanley, Henry Morton: Mr Stanley's Mission. The River Livingstone, A Land of Ivory Houses, The Thirty-Two Battles. To the Editors of the ,Daily Telegraph' and ,New York Herald. ', Loanda, Sept. 1, 1877. In: The Daily Telegraph vom 22.11.1877. 
Phantasma der Belletristik im (seriellen) Abenteuerroman (z. B. H. Rider Haggards King Solomon's Mines, 1885, und Allan Quatermain, 1887, sowie Arthur Conan Doyles The Lost World, 1912). ${ }^{6}$ Diese Tradition bildet den textuellen Hintergrund, vor dem die Schriften der primatologischen Autor/innen sich bewegen, wenn sie sich (auch) an ein populärwissenschaftliches Publikum richten. Zweitens scheinen die Afrika-, in kleinerem Umfang auch die Asienreisenden nach der Veröffentlichung von Darwins On the Origin of Species (1859) besessen davon, die großen Affen aufzuspüren und wenn möglich neue Arten zu finden. Somit prägen die betreffenden Reise- und Forschungsberichte als Textbelege der Begegnung mit fremden Spezies auch ein Phantasma vom Erstkontakt und das Bild der großen Affen mit. Drittens können diese Berichte als Vorlagen eines emotionalen (Selbst-) Erlebens betrachtet werden und dienen somit als frühe Modelle für die Affektivität von Forscher/innen im Feld.

Zentral dafür ist in den Reiseberichten das Narrativ von der Suche nach dem Evolutionstier. ${ }^{7}$ Dieses Narrativ lässt sich in zwei Ausprägungen finden: In seiner einen Form soll die Sammelleidenschaft und das Engagement der Forschenden untermalt werden. Ein Beispiel hierfür ist Alfred Russel Wallace' 1865 veröffentlichter Bericht The Malay Archipelago. Im Untertitel wird dieser Bericht bereits als A Narrative of Travel, With Studies of Man and Nature bezeichnet. ${ }^{8}$ Das andere Narrativ des Reiseberichts ist getrieben von der Jagdleidenschaft des ,Great White Hunter', wie er als kulturelle Figur im Rahmen der Safari später formiert wird, aber bereits in viktorianischen Reiseberichten und der kulturellen Imagination der Abenteuerliteratur des ausgehenden 19. Jahrhunderts präfiguriert ist. ${ }^{9}$ Das am häufigsten literarisierte Beispiel hierfür ist sicherlich Henry Morton Stanley. Noch bevor Stanley jedoch auch nur den ersten Fuß auf den Kontinent gesetzt hatte, war Paul Du Chaillu 1861 mit seinen Explorations and Adventures in Equatorial Africa bereits mit, wie sein Untertitel verrät, Accounts of the Manners and Customs of the People, and of the Chace of the Gorilla, Crocodile, and other Animals berühmt geworden. ${ }^{10}$

\footnotetext{
${ }^{6}$ Haggard, H. Rider: King Solomon's Mines. London 1885; Haggard, H. Rider: Allan Quatermain. London 1887; Doyle, Arthur Conan: The Lost World. London 1912.

${ }^{7}$ Dass die Faszination für die großen Affen als Brückentiere zwischen Mensch und Tierreich der Postulation der Evolutionstheorie lange vorausgeht, bzw. sich Letztere in Ersterer bereits ankündigt, beschreibt Borgards: Menschenaffen/Affenmenschen.

${ }^{8}$ Wallace, Alfred Russel: The Malay Archipelago: The Land of The Orang Utan and The Bird of Paradise. A Narrative of Travel, With Studies of Man and Nature. 2 Bde. London 1869. Im Folgenden wird nur aus dem ersten Band zitiert und dieser, wenn zur Unterscheidung nötig, als $M A$ geführt; Seitenangaben im Text.

${ }^{9}$ Vgl. Herne, Brian: White Hunters. The Golden Age of African Safaris. New York 1999; Mandiringana, E./ Stapleton, Timothy J.: The Literary Legacy of Frederick Courteney Selous. In: History in Africa 25 (1998), S. 199-218.

${ }^{10} \mathrm{Du}$ Chaillu, Paul: Explorations and Adventures in Equatorial Africa with Accounts of the Manners and Customs of the People, and the Chase of the Gorilla, Crocodile, and other Animals. New York 1861. Im Folgenden, wenn zur Unterscheidung nötig, als EA geführt; Seitenangaben im Text.
} 
Der Brite Wallace und der Franko-Amerikaner Du Chaillu sind den zeitgenössischen Konventionen des (Forschungs-)Reiseberichts verschrieben: Sie sind bemüht, sowohl zu informieren und berichten, als auch zu unterhalten und sich zu rechtfertigen. ${ }^{11}$ Beiden ist daran gelegen, ihr Publikum bereits im Titel und in der visuellen Gestaltung der Publikation darauf hinzuweisen, welche Tiere hier neu bzw. erstmals ausführlich beobachtet, gesammelt und dargestellt werden. Bei Du Chaillu wird der Gorilla in Standbein-Spielbein-Pose als Frontispiz an prominente Stelle gerückt (Abb. 2.1); Wallaces Erstausgabe wird von einem Orang-Utan-Kopf in Gold auf dem Buchdeckel geschmückt (ähnlich wie Abb. 2.2). und zeigt einen anderen Orang-Utan als Frontispiz des ersten Bandes.

Beide messen den Erfolg ihrer Expeditionen und Forschungsaufenthalte daran, wie viele Spezies entdeckt und Spezimina heimgeschickt werden konnten. ${ }^{12} \mathrm{Sie}$ verstehen sich ebenfalls beide als privilegierte Naturkundler; doch während Wallace sich schlicht als ,naturalist“ (MA, 54) bezeichnet, ist Du Chaillu ein ,ardent naturalist" ( $E A, v)$. Sie zeigen in ihren Berichten aber auch das emotionale Spektrum auf, welches innerhalb der Genre-Konventionen der Begegnung mit Affen zur Verfügung stand: Worin sich Wallace und Du Chaillu wesentlich unterscheiden, ist die emotionale Färbung ihrer Reiseschilderungen und ihrer Affenbegegnungen. Wallace ist der fast pathologisch nüchterne, gefühlskalte Sammler, du Chaillu der sentimentale Jäger der affektiven Emphase.

Bereits in den jeweiligen Prefaces, mit denen Wallace und Du Chaillu sich und das für ihre Reiseberichte zentrale Thema ihrem Publikum vorstellen, ihre Reise zusammenfassen und ihr Anliegen schildern, treten diese Unterschiede zutage. Paul Du Chaillus Explorations and Adventures umfasst seine Reise aus den Jahren 1856-1858 in das äquatoriale Afrika, eine Region, „remarkable chiefly for its fauna“, ,in many respects not only extraordinary, but peculiar" (v, Hervorheb. MS). Wie bemerkenswert diese Fauna für Du Chaillu ist, zeigt schon der zweite Satz seiner Preface: „In this comparatively narrow belt is found that monstruous

\footnotetext{
${ }^{11}$ So schreibt Du Chaillu z. B., er habe auf die Schilderung eines verzweigten Delta-Sumpflands verzichtet, „because it was extremely barren of incidents interesting to the reader" (EA, S. vi-vii). Wallace fühlt sich berufen, die lange Zeit zwischen der Rückkehr nach England 1862 und der Veröffentlichung des Reiseberichts 1869 zu rechfertigen: „[M]y weak state of health“ und „the unpacking, sorting, and arranging of such a mass of specimen“ (MA, S. vii) habe ihn in Anspruch genommen. Wissenschaftliche Gründlichkeit sei ihm wichtiger gewesen als sofortiger Ruhm: „I could indeed, at once have printed my notes and journals, leaving all references to questions of natural history for a future work; but I felt that this would be as unsatisfactory to myself, as it would be disappointing to my friends, and uninstructive to the public" (S. viii).

${ }^{12}$ Wallace verzeichnet „125.660 specimen of natural history“ (MA, S. xiii), deren Verbleib in naturgeschichtlichen Museen und Sammlungen er stolz dokumentiert (,,and I prepared a perfect skeleton, which was afterwards purchased for the Derby Museum“, S. 64); Du Chaillu verweist auf mehr als 2000 Vögel und ca. 200 „,quadrupeds“ samt 80 Skeletten, für die er verantwortlich zeichnen könne (EA, S. x).
} 


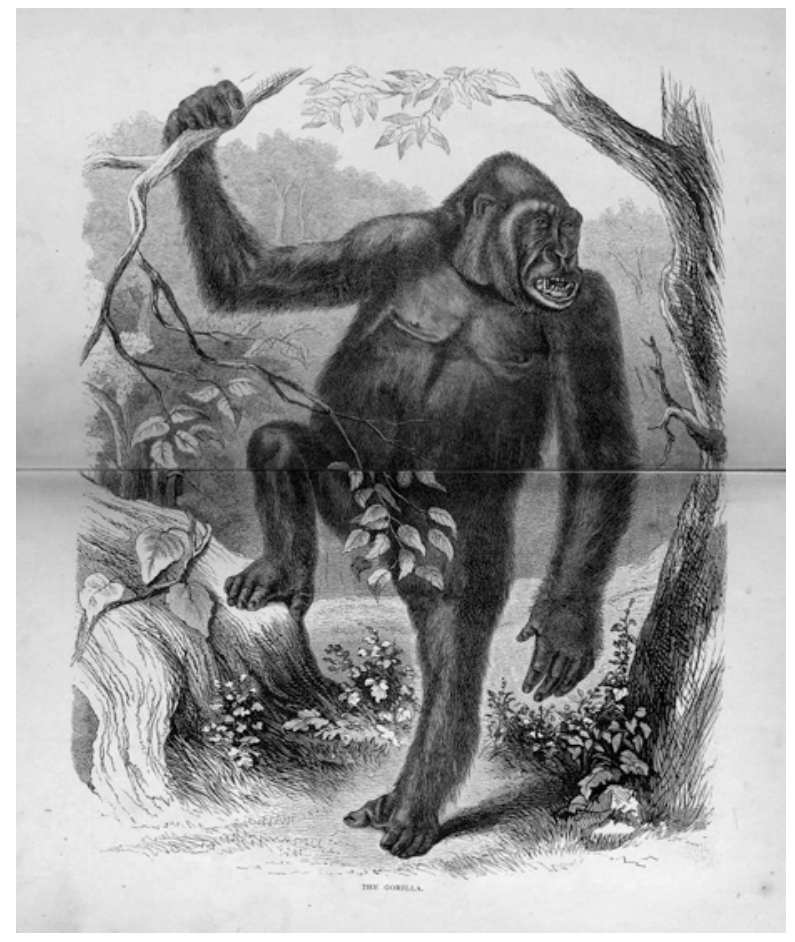

Abb.2.1 Frontispiz, Paul Du Chaillu: Explorations and Adventures in Equatorial Africa. (Quelle: Wikimedia commons; https://commons.wikimedia.org/wiki/File:The_Gorilla_-_Du_ Chaillu.jpg)

and ferocious ape, the gorilla“ (v). Damit ist der große Affe als wildes Monster eingeführt. Die Emphase dieses Satzes entsteht durch ein Demonstrativpronomen (,that ape'), welches die Kenntnis dieses Affen auch bei den Leser/innen bereits voraussetzt, sodass dessen nachgestellte Nennung (,the gorilla') bereits einen Fundus an Vorstellungen mitaktiviert. ${ }^{13} \mathrm{Um}$ die Bestialität zu verstärken wird dem Gorilla polarisierend ein anderer Affe gegenübergestellt: „Here, too, and here only, is the home of the very remarkable nest-building ape, the Troglodytes calvus,

\footnotetext{
${ }^{13}$ Der Gorilla besitzt zu dieser Zeit bereits einen festen Platz im Evolutionsdiskurs durch die von Richard Owen und Thomas Henry Huxley vorangetriebene Kontroverse um den Gorilla als menschenähnlichstem Affen. Vgl. Griem: Monkey Business, S. 81 ff. Nach der Theoriebildung am ersten vollständigen Gorilla-Skelett, das 1851 in Europa zur Verfügung stand, ist es dann vor allem Du Chaillu, der Munition für diese Kontroverse liefert, indem er auf seiner Afrikareise auch den ,missing link'-Topos aufnimmt und sowohl die ,Schwarzheit' des Gorillas betont als auch ein reales Brückentier zwischen afrikanischer Bevölkerung und Menschenaffe ausschließt. Vgl. Richter: Literature after Darwin, S. 34 ff.
} 


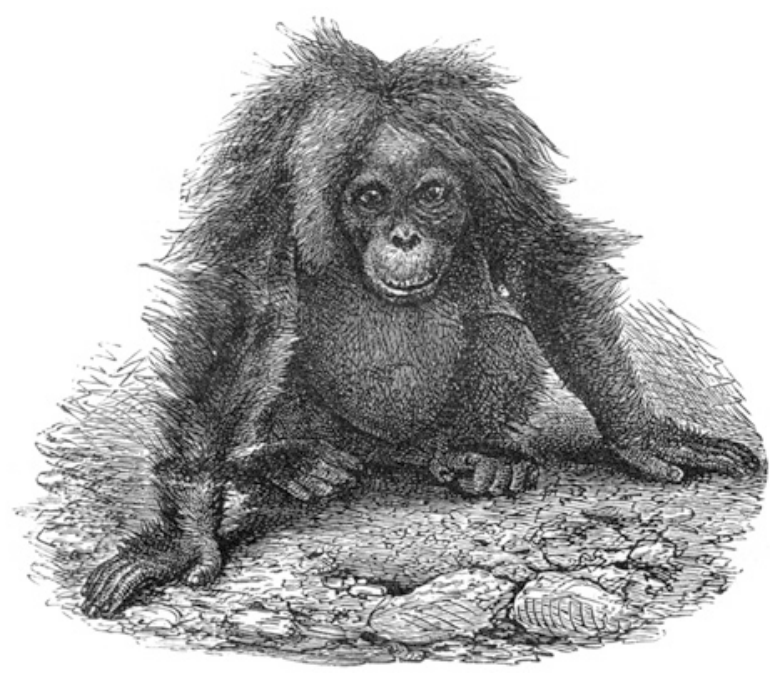

Abb.2.2 „Female Orang-Utan“; Alfred Russel Wallace: The Malay Archipelago (London ${ }^{10} 1890$ ). (Quelle: Wikimedia commons; https://commons.wikimedia.org/wiki/File:Malay_ Archipelago_Female_Orangutan.jpg)

the nshiego mbouve of the natives" (v, Hervorheb. im Original). ${ }^{14}$ Die Konfrontation des Monströsen mit dem heimelig Nestbauenden erscheint hier wie zufällig in einer Auflistung, die auch noch „,another ape“, den „hitherto unknown koolookamba“ (v, Hervorheb. im Original), und den Schimpansen umfasst. Sie ist jedoch programmatisch für Du Chaillus Inszenierung des Gorillas als schreckliche Kreatur und damit auch als singulär ebenbürtiger Gegner in einem so metaphorischen wie pragmatischen Kampf ,Mann gegen Affe‘. Denn, wie diese Linie im ersten Kapitel der Explorations and Adventures weitergeführt wird, „the fierce, untamable gorilla" (25, Hervorheb. im Original) ist einzigartig unter den Affen, da er menschenähnlich und doch von unbezwingbarer Wildheit und - zumindest für die ,Eingeborenen “ - schreckenseinflößend ist: „that remarkable ape which approaches nearest, in physical conformation and in certain habits, to man, and whose unconquerable ferocity has made it the terror of the bravest native hunters" ( $25 \mathrm{f}$.).

Ganz anders dagegen führt Wallace seinen Affen in The Malay Archipelago, seinem Bericht über die naturkundliche Erforschung der gleichnamigen südostasiatischen Inselgruppe von 1854 bis 1862, ein. Zunächst lächelt wie erwähnt ein junger Orang-Utan-Kopf vom Buchdeckel und wird sogleich mit dem Frontispiz, auf dem ein ausgewachsener Orang-Utan einem indigenen Dyak die Zähne ins Fleisch schlägt (Abb. 2.3), kontrastiert - und dann eine Weile vergessen.

\footnotetext{
${ }^{14} \mathrm{Vgl}$. zu einer kontinuierlichen Polarisierung zwischen dem Tierlich-bestialischen und dem Anthropomorphen bei du Chaillu Griem: Monkey Business, S. 85.
} 


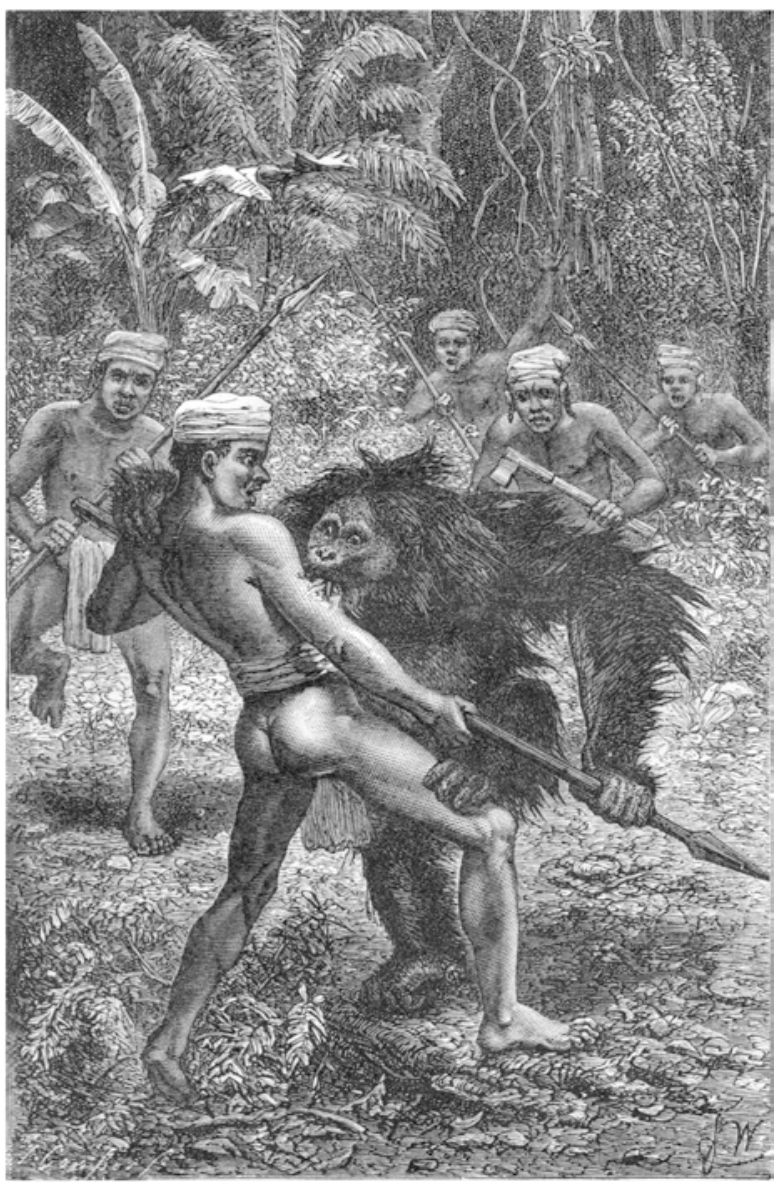

Abb. 2.3 Frontispiz „Orang-utan attacked by Dyaks“, Alfred Russel Wallace: The Malay Archipelago, Bd. 1. (Quelle: Wikimedia commons; https://commons.wikimedia.org/wiki/ File:Malay_Archipelago_Orang-Utan_attacked_by_Dyaks.jpg)

Wallace' Preface widmet sich der Zeitverzögerung der Veröffentlichung, einer üppigen Kontemplation seiner zahlreichen mitgebrachten und an Museen verkauften Exponate, der inhaltlichen Anordnung und Illustration seines Textes, seiner geographischen Ordnung des bereisten Archipels, einem Kommentar zu „the principles developed by Mr. Darwin“ (xii) $)^{15}$ und einer tabellarischen Auflistung der von ihm präparierten Spezimina. Die einzigen Tiere, die hier emphatisch erwähnt werden, sind „my large collection of Longicorn beetles“ (ix). Vom

\footnotetext{
${ }^{15}$ Wallace entwickelte zeitgleich und zunächst unabhängig von Charles Darwin ebenfalls die Idee einer Evolution der Arten auf Basis natürlicher Selektion; Wallace' On the Tendency of Varieties wurde gemeinsam mit einigen Schriften Darwins 1858 vor der Linnean Society of London
} 
Orang-Utan findet sich zunächst keine Spur. Auch das eigens dem Orang-Utan gewidmete Kapitel IV, Borneo - The Orang-Utan (54 ff.), zeigt den Naturkundler erst einmal mit Blick auf den Boden statt in die Baumwipfel: „During my first walk along this road I saw few insects or birds“, bedauert er, „but noticed some very handsome orchids in flower" (55). Wallace' Hauptinteresse gilt den Insekten, und er hat Glück: „I never enjoyed such advantages in this respect“, sodass er binnen zwei Wochen und mithilfe der Mienenarbeiter, denen er einen Cent pro Exemplar zahlt, die Anzahl seiner bislang gesammelten Käfer verdoppeln kann. Wallace entbehrt überwiegend eines emotionalen Vokabulars, doch der Raum, der der Sammeltätigkeit von Insekten eingeräumt wird - drei Seiten allein zu Beginn dieses Kapitels (56-59) -, spricht für die große Faszination, die den Käfer-Enthusiasten hier noch im Nachhinein im Prozess der Verschriftlichung bestimmt. Erst auf Seite 61 kommt er schließlich dazu, den titelgebenden Affen vorzustellen:

\footnotetext{
One of my chief objects in coming to stay at Simunjon was to see the Orang-utan (or great man-like ape of Borneo) in his native haunts, to study his habits, and obtain good specimen of the different varieties and species of both sexes, and of the adult and young animals. In all these objects I succeeded beyond my expectation, and will now give some account of my experiences in hunting the Orang-utan, or ,Mias', as it is called by the natives; and as this name is short, and easily pronounced, I shall generally use it in preference of Simia Satyrus, or Orang-utan. (61 f.)
}

Wallace' Orang-Utan zeichnet sich vor allem dadurch aus, dass er, wie hier in Klammern in die bereits auf den enzyklopädischen Eintrag hingedachte Textpassage eingefügt, der „great man-like ape“ Borneos ist. In dieser Umschreibung wird die Menschenähnlichkeit zur Bestimmung des Affen. Seine anderen Bezeichnungen, das allgemeine „Orang-utan“, ${ }^{16}$ die zeitgenössische taxonomische Bezeichnung „Simia Satyrus“, und das einheimische „Mias“ dienen der Vollständigkeit, ebenso wie die Aufzählung der ,different varieties and species of both

verlesen und in der Proceedings-Reihe der Linnean Society veröffentlicht. Vgl. Darwin, Charles R./Wallace, Alfred Russel: On the Tendency of Species to form Varieties; and on the Perpetuation of Varieties and Species by Natural Means of Selection. In: Journal of the Proceedings of the Linnean Society of London. Zoology 3 (1858), S. 46-55. Darwin und Wallace - zu der Zeit noch auf Reisen - standen seit spätestens 1857 in Korrespondenz, vgl. Marchant, James (Hg.): Alfred Russel Wallace. Letters and Reminiscences. Bd. 1. London 1916, S. 66. Wie stark der Austausch der beiden Denker der Evolutionstheorie war, zeigt sich nicht zuletzt daran, dass Wallace sein Malay Archipelago Darwin widmet: „NOT ONLY/ AS A TOKEN OF PERSONAL ESTEEM AND FRIENDSHIP/ BUT ALSO/ TO EXPRESS MY DEEP ADMIRATION/ FOR/ His Genius and his Works" (ohne Seitenzahl).

${ }^{16}$ Der seit dem 17. Jahrhundert in Europa verwendete Begriff ,Orang-utan“ wird häufig als originär malayische Bezeichnung des Affen als ,Wald-Mensch“ angegeben. Dellios weist aber darauf hin, dass die beiden einzelnen Lexeme zwar dem malayischen Wortschatz entstammen, das betreffende Tier jedoch im Malayischen ,Mawas' genannt werde. Siehe Dellios, Paulette: A Lexical Odyssey from the Malay World. In: Studia Universitatis Petru Maior - Philologia 4 (2005), S. 141-144. 
sexes, and of the adult and young animals“ (wesentlich also: alle Orang-Utans). Wallace' selbsterklärte Bestimmung ist es nun, dieses Tier zu erforschen. Dabei beschreibt er seine Zielsetzung - das Sammeln - bereits so ausführlich, dass sie als eigene elaborierte Methode erscheint.

Diese ,Methode' des naturkundlichen Sammelns ist bei Wallace und Du Chaillu im Wesentlichen die Gleiche: Tiere, besonders vermutete neue Arten, werden wie in der zur Unterhaltung dienenden Jagd aufgespürt, beobachtet und geschossen, um dann als ,Specimen' nach taxidermischer Arbeit in naturhistorische Sammlungen eingefügt zu werden: „to see [...] in his native haunts, to study his habits, and obtain good specimen“ (MA, 61); ,[to] acquaint myself alike with the haunts and habits of the gorilla“ $(E A, 26)$, sind erklärte Ziele beider Autoren. Die Reiseberichte sind ,some account of my experience in hunting the Orangutan“ (MA, 62) und „Accounts [...] of the Chase of the Gorilla“ (EA, Titel). Dieses methodische Vorgehen - Jagd bzw. Verfolgung, Beobachtung und Tötung des Tieres - entfaltet sich in einer dramatischen Struktur, deren Höhepunkte in der Begegnung mit dem Tier und in der Tötungsszene zu finden ist. Ein solches „drama of the safari“"17 beerbt die bloße Jagd als Modell, wie Donna Haraway darlegt und anhand des 60 Jahre später tätigen Carl Akeley illustriert:

Ideally, the killing itself had to be accomplished as a sportsmanlike act. Perfection was heightened if the hunt were a meeting of equals. So there was a hierarchy of game according to species [...]. The gorilla was the surpreme achievement, almost a definition of perfection in the heart of the garden at the moment of origin. Perfection inhered in the animal itself, but the fullest meanings of perfection inhered in the meeting of animal and man, the moment of perfect vision, of rebirth. Taxidermy was the craft of remembering this perfect experience. $^{18}$

Dass der Gorilla im idealen Drama der Jagd als Safari zu Beginn des 20. Jahrhunderts, wie es Haraway beschreibt, eine solche übergeordnete Rolle spielt, liegt wesentlich daran, dass Du Chaillu ihn Mitte des 19. Jahrhunderts als begehrte Bestie inszeniert und dieses Drama affektiv ausgestaltet.

\subsubsection{Der sentimentale Jäger Paul Du Chaillu}

Du Chaillus erste Begegnung mit einem Gorilla, dem Tier, dem er in seinem Bericht bereits seit einiger Zeit im wörtlichen Sinne auf der Spur ist, findet statt, als er sich hungrig mit seinen Männern durch Zuckerrohrbüschel schlägt: „But as we were plucking my men perceived what instantly threw us all into the greatest excitement“ (84). Wahrnehmung und Affizierung sind hier unmittelbar miteinander verbunden. Aus dieser affektiven Wahrnehmung resultiert eine Erkenntnis und dieser folgt wiederum eine differenzierte Emotion: „I knew that

\footnotetext{
${ }^{17}$ Haraway: Primate Visions, S. 40.

${ }^{18}$ Haraway: Primate Visions, S. 41.
} 
these were fresh tracks of the gorilla, and joy filled my heart" (84, Hervorheb. MS). Der Anblick der Gorilla-Spur aktiviert den Jäger umgehend physisch und kognitiv; die Wirkung ist dabei affektiv:

\begin{abstract}
We followed these traces, and presently came to the footprints of the so-long desired animal. It was the first time I had ever seen these footprints, and my sensations were indescribable. Here was I now, it seemed, on the point of meeting face to face that monster of whose ferocity, strength, and cunning the natives had told me so much; an animal scarce known to the civilized world, and which no white man before had hunted. My heart beat till I feared its loud pulsations would alarm the gorilla, and my feelings were really excited to a painful degree. (84, Hervorheb. MS)
\end{abstract}

Die subjektive Wahrnehmung des Reisenden Du Chaillu bildet zwar überwiegend den Fokus von Explorations and Adventures, beispielsweise im Registrieren sowohl der körperlichen wie affektiven Phänomene. Jedoch wird von diesen häufig humoristisch distanziert berichtet. Großen Hunger bei gleichzeitigem Ekel vor den Essgewohnheiten der Einheimischen kann er zuspitzen auf das selbstironische Fazit: „,T] he snake was eaten, and I, the only empty-stomached individual of the company, had sufficiently reflected on the disadvantages of being bred in a Christian country" (83). In der obigen Passage jedoch stehen Du Chaillus Empfindungen (,my sensations“) ganz explizit und unironisch im Mittelpunkt, und obwohl er sie als ,indescribable“ bezeichnet, ist er dennoch darum bemüht, die Empfindungsepisode für seine Leser/innen nachvollziehbar, wenn nicht sogar nachfühlbar werden zu lassen. So erläutert er die Bedeutung der Situation und ruft erneut den Mythos des begehrten (,so-long desired“) Gorillas auf: „Here was I now $[\ldots]$ on the point of meeting face to face that monster $[\ldots][$,$] an animal [\ldots]$ which no white man before had hunted" (84). Der Affe erscheint wieder als Monster, dessen Eigenschaften, Wildheit, Stärke und Gerissenheit, seine Potenz und seinen Wert als Gegenüber in der Jagd erhöhen. Du Chaillu registriert an dieser Stelle sein körperliches Empfinden: „My heart beat till I feared its loud pulsations would alarm the gorilla" (84). In einem Stufenmodell der affektiven Erregung scheint Du Chaillu ganz oben angekommen zu sein, wenn er schreibt „my feelings were really excited to a painful degree" (84). Die am Gorilla entzündete Empfindungsintensität äußert sich in einem die Schmerzgrenze durchbrechenden Herzklopfen und wird begleitet von der Sorge, diese (Über-)Emotionalität könne als wahrnehmbare körperliche Reaktion auch den Gorilla, die Beute, aktivieren. ${ }^{19}$

\footnotetext{
${ }^{19}$ Diese physisch-affektive Selbstbeschreibung folgt einem Muster der affektiven und physiologischen Wirkung bzw. Bedeutung der Jagd: Sie ähnelt folgender Selbstauskunft eines „Southern, wealthy, white“ Jägers im Rahmen einer Autoethnographie der Jagd: „There is an overwhelming intensity of feeling in the instant of killing. After hours and even months of anticipitation and rising action, the kill constitutes the critical moment. Physiologically, my veins are subject to a massive torrent of hand-trembling adrenaline. The deluge is such that I wonder if each deafening pound in my chest that reverberates across my body may actually be my heart exploding. And more". Presser, Lois/Taylor, William V.: An autoethnography of hunting. In: Crime, Law and Social Change 55/5 (2011), S. 483-494, hier S. 489 f.
} 
Die bemerkenswerte Affizierung und Du Chaillus Emphase in deren Wahrnehmung werden im Text implizit abgegrenzt von einem Verdacht einer unangemessenen, da weiblichen Emotionalität. ${ }^{20}$ Männliche und weibliche Reaktionen auf den Gorilla werden in den Explorations aufgrund ihrer spezifischen Jagd-Konstellation gegensätzlich entworfen. So gibt Du Chaillu an, man habe die Frauen des Reisetrupps zurücklassen müssen, denn: „The women were terrified, poor things“ (84), ,with fear written on their faces“ (84), ,a lively fear of the terrible gorilla, in consequence of various stories current among the tribes, of women having been carried off into the woods by the fierce animal“" (85). Im Gegensatz zu den durch den Affen als Jäger in außerordentlichen Schrecken versetzten Frauen ist Du Chaillus emotionales Erleben hinsichtlich des Affen als Beute durchweg positiv. Die Erwähnung der Frauen dient aber nicht allein der emotionalen Positionierung Du Chaillus sondern auch der des Affen: Als weitere Charakterisierung (der Wirkung) des monströsen Affen unterstützt sie den Spannungsaufbau hinsichtlich einer zu erwartenden klimaktischen Begegnung. Das immer noch nicht sichtbare Tier wird in dieser Szene weiter mit Eigenschaften und Beschreibungen besetzt, die es als ultimativen Gegner aufwerten:

[T] he gorilla gives you no time to reload, and woe to him whom he attacks! We were armed to the teeth. [...] [F]or the male gorilla is literally the king of the African forest. He and the crested lion of Mount Atlas are the two fiercest and strongest beasts of this continent. The lion of South Africa can not compare with either for strength or courage. (84)

For years I had heard of the terrible roar of the gorilla, of its vast strength, its fierce courage if, unhappily, only wounded by a shot. [...] [W]e were to pit ourselves against an animal which even the tiger of these mountains fears, and which, perhaps, has driven the lion out of this territory [...]. (85)

Folglich wappnet man sich, formt strategische Gruppen und beginnt die Jagd. Du Chaillu rekonstruiert anhand der Spuren die Anzahl, das Aussehen und Verhalten der Tiere:

As we followed the tracks we could easily see that there were four or five of them; though none appeared very large. We saw where they had run along on all fours, the usual mode of progression of these animals, and where, from time to time, they had seated themselves to chew the canes they had borne off. (84 f.)

Das interessierte Forschersubjekt und der affizierte Jäger konfundieren hier in der Feststellung: ,Thus it was with no little emotion that I now turned again toward

\footnotetext{
${ }^{20}$ Zur „geschlechterspezifischen Typisierung der Empfindsamkeit“ durch eine ,paradigmatische Kodifizierung der weiblichen Sonderanthropologie“ seit dem letzten Drittel des 18. Jahrhunderts vgl. Honegger, Claudia: Die Ordnung der Geschlechter: Die Wissenschaften vom Menschen und das Weib 1750-1850. Frankfurt a. M. u. a. 1991, S. 165 ff., 182 ff. Zur lexikalischen Gestaltung der geschlechterspezifische Differenzierung von (Mit-)Gefühl im Rahmen einer Neujustierung des Verhältnisses von Vernunft und Gefühl im 19. Jahrhundert vgl. Frevert, Ute: Gefühle definieren, S. $35 \mathrm{ff}$.
} 
the prize at which I had been hoping for years to get a shot" (85, Hervorheb. MS); „The chase began to be very exciting“, ja sogar gesteigert: „I confess that I never was more excited in my life" (85). Wieder werden diese Emotionen verstärkt durch den Resonanzkörper seiner Männer (,my men“, 85), denn „they were in even greater excitement than myself“" (85).

Man pirscht sich an die Gorillas heran, bis es zum Erstkontakt kommt. Für die Gorillas ist dieser Erstkontakt markiert durch einen die Perspektive wechselnden Blick („The watchful animal saw him“, 85), für die Menschen zunächst durch ein signifikantes Geräusch: „a strange, discordant, half human, devilish cry“ (85). Dann sind vier Jungtiere auf der Flucht zu sehen. Du Chaillus Sätze werden hier kürzer, der Rhythmus ist beschleunigt, um die Anspannung der Szene zu vermitteln: „We fired, but hit nothing. Then we rushed on in pursuit; but they knew the woods better than we. [...] We ran till we were exhausted, but in vain" (86). Zurück im Camp evaluiert Du Chaillu diesen Erstkontakt - wieder mit Hinweis auf seine Gefühlslage:

I protest I felt almost like a murderer when I saw the gorilla this first time. As they ran on their hind legs - they looked fearfully like hairy men; their heads down, their bodies inclined forward, their whole appearance like men running for their lives. Take with this their awful cry, which, fierce and animal as it is, has yet something human in its discordance, and you will cease to wonder that the natives have the wildest superstitions about these, wild men of the woods'. (86, Hervorheb. MS)

Noch hat Du Chaillu keinen der Affen geschossen und doch ist er bereits mit seinem moralischen Empfinden beschäftigt. Hervorgerufen wird dies durch die Ambiguität, welche den Gorilla zum ebenbürtigen Gegner, Haraways „surpreme achievement“ ${ }^{21}$ in der als Kampf konzipierten Jagd macht: „,[T]hey looked fearfully like hairy men"; ,,animal as it is" hat der Gorilla „,yet something human in its discordance" (86). Später wird diese Ambiguität noch expliziter affektiv unterlegt: „Though there are sufficient points of diversity between this animal and man, I never kill one without having a sickening realization of the horrid human likeness of the beast" (323, Hervorheb. MS). Menschenähnlichkeit und Gefühlsintensität provozieren hier eine intime, eine körperlich affizierende Nähe, und die daraus resultierende Reaktion ist eine des Ekels als erkenntnisstiftender elementarer Affekt: ein Alarm- und Ausnahmezustand, der aus der Erfahrung einer ungewollten Nähe resultiert und der als Reaktion auf das Abjekte zu verstehen ist, welches als Dazwischen, als Ambiges und Gemischtes Identitäten, Systeme und Ordnungen (ver-)stört. ${ }^{22}$

Seine Klimax findet dieses Dilemma des Fühlens in einer wenige Seiten später folgenden Passage: in der Begegnung mit einem ausgewachsenen Gorilla,

\footnotetext{
${ }^{21}$ Haraway: Primate Visions, S. 41.

${ }^{22} \mathrm{Vgl}$. Menninghaus, Winfried: Ekel. Theorie und Geschichte einer starken Empfindung. Frankfurt a. M. 1999, S. 7; Kristeva, Julia: Powers of Horror. An Essay on Abjection. New York 1982 (frz. 1980), S. 4.
} 
Du Chaillus erstem Exemplar. Auch diese wird wieder szenisch gestaltet, wie hingedacht auf eine filmische Umsetzung, und soll daher hier in Gänze wiedergegeben werden. Die Passage beginnt ebenfalls mit einem signifikanten Schrei des Gorillas:

And presently I noticed, ahead of us seemingly, a noise as some one breaking down branches or twigs of trees. [...] Suddenly, as we were yet creeping along, in a silence which made a heavy breath seem loud and distinct, the woods were at once filled with the tremendous barking roar of the gorilla.

Then the underbrush swayed rapidly just ahead, and presently before us stood an immense male gorilla. [...] [W] hen he saw our party he erected himself and looked us boldly in the face. He stood about a dozen yards from us, and was a sight I think never to forget. Nearly six feet high [...], with immense body, huge chest, and great muscular arms, with fiercely glaring large deep gray eyes, and a hellish expression of face, which seemed to me like some nightmare vision: thus stood before us this king of the African forest.

He was not afraid of us. He stood there, and beat his breast with his huge fists till it resounded like an immense bass-drum, which is their mode of offering defiance; meantime giving vent to roar after roar. [...] His eyes began to flash fiercer fire as we stood motionless on the defensive, and the crest of short hair which stands on his forehead began to twitch rapidly up and down, while his powerful fangs were shown as he again sent forth a thunderous roar. And now truly he reminded me of nothing but some hellish dream creature - a being of that hideous order, half man half beast, which we find pictured by old artist in some representation of the infernal region. He advanced a few steps then stopped to utter that hideous roar again - advanced again - and finally stopped when at a distance of about six yards from us. And here, as he began another of his roars and beating his breast in rage, we fired, and killed him.

With a groan which had something terribly human in it, and yet was full of brutishness, it fell forward on its face. The body shook convulsively for a few minutes, the limbs moved about in a struggling way, and then all was quiet - death had done its work, and I had leisure to examine the huge body. (S. 98 ff.)

Das Tier scheint durch diese Szene zunächst individualisiert: mit einer beeindruckenden Physis, einem signifikanten Schrei (,the most singular and awful noise heard in these African woods“, 98, „that hideous roar“, 101), einem Gesicht und Gesichtsausdruck (,hellish expression of a face“, 100), der Du Chaillu das Fürchten lehrt. Gleich mehrfach vergleicht er die Szene und den Gorilla mit einem Alptraum (,a nightmare vision“, 100). Es ist nicht irgendein Alptraum, denn als „hellish dream creature“ (101) ist ihm der Gorilla eine grauenerregende Figur aus einer frühneuzeitlichen Höllendarstellung - „hellish“ und ,hideous“ fungieren hier als Signalwörter des Infernalen -, denn in seiner imposanten Erscheinung kann er nur einer gefährlichen, alptraumhaften, hybriden Kategorie angehören: ,a being of that hideous order, half man half beast" (101). Passenderweise lässt Du Chaillu den Gorilla hier alliterarisch Feuer zeigen, seine Augen „flash fiercer fire“ (101), während Du Chaillu und seine Gefolgsleute starr vor Angst (,motionless on the defensive“, 101) verharren angesichts der Kreatur im Affen. Gleichzeitig wird der Gorilla nicht nur als individueller, selbst emotional äußerst aktivierter (,beat his breast in rage“, 98) Gegner gezeichnet, sondern auch als Vertreter seiner Art ethnisiert: Sein Gebrüll klinge wie ,an immense bass-drum“ und sei 
„their mode of defiance“ (98, Hervorheb. MS) heißt es. Hier taucht die Opposition von „we“ und „they“ wieder auf, die schon beim Erstkontakt mit den Gorillas anklang (,they knew the woods better than we“, 86). Dieses textuelle Othering dient ebenso wie das Utensil der Trommeln als festes Inventar afrikanischer, Customs and Manners': Der Modus ist hier als Verweis auf ein ,éthnos' lesbar. ${ }^{23}$ Doch die Demarkierung der Differenz wird implizit vergleichend verschoben: von der Ebene der Differenz zwischen der christlich-zivilisierten und der indigenbarbarischen Welt, die Du Chaillu immer wieder in den Explorations humoristisch bemüht, auf die Ebene der Differenz zwischen Mensch und Tier.

Diese unterschiedlichen Teile einer funktional deskriptiven, zugleich emotionsgeleiteten und emotionalisierenden Anthropomorphisierung des Gorillas als ethnisiertem Individuum und als Typus der alptraumhaften Höllenfigur kulminieren in dieser Szene im Tod des großen Affen. Hier wird der Gorilla, mit einem Laut, „terribly human“, ,yet [...] full of brutishness“ (101), zu einem ,it“ in dem Moment, in dem er auf sein - so menschenähnliches - Gesicht fällt. Diese Szene lässt sich im Kontext von Emmanuel Levinas' Ethik lesen, die Verantwortung und Schuld in der Von-Angesicht-zu-Angesicht-Begegnung mit dem Anderen beginnen lässt. ${ }^{24}$ Das Gesicht macht für Levinas den Menschen nicht nur aus, ${ }^{25}$ aus ihm spricht auch das durch diese Bedingung evozierte ,Du wirst [mich] nicht [ungestraft] töten' ${ }^{26}$ Indem der Gorilla auf das Gesicht fällt, wird die Möglichkeit und Notwendigkeit des (An-)Erkennens des Anderen in seiner Andersheit ${ }^{27}$ dem bereits durch die Einschreibungen in einen kunsthistorischen und mythischen Kanon kategorial vorgebeugt werden sollte - aufgehoben bzw. verdeckt. Dieses nicht-menschliche Tier, ,facing us as few animals dare face man" (323, Hervorheb. MS), ist das Kategorien sprengende Andere. Das Angebot der Einsicht in die ambige epistemologische Andersheit des Gorillas wird bei Du Chaillu als alptraumhafte Bedrohung verstanden und wohl auch als Machteinbuße empfunden. Mit dem Schuss in die Brust wird das Gesicht, welches die Kraft besaß, affektiv und appellativ das ,vouloir tuer“28 einzuschränken, zugleich bewahrt und

\footnotetext{
${ }^{23} \mathrm{Vgl}$. zum textuellen Othering moderner Anthropologie und ihrer Vorläufer im ,Customs and Manners'-Bericht mittels abstrahierender Personalpronomen Pratt, Mary Louise: Scratches on the Face of the Country, or, What Mr. Barrow Saw in the Land of the Bushmen. In: Critical Inquiry 12/Autumn (1985), S. 119-143, hier S. $120 \mathrm{ff}$.

${ }^{24} \mathrm{Vgl}$. Levinas, Emmanuel: Totalität und Unendlichkeit. Versuch über die Exteriorität. Freiburg/ München 1987 (frz. 1961), S. 190-112, 267 ff.; Stegmaier, Werner: Emmanuel Levinas zur Einführung. Hamburg 2009, S. $134 \mathrm{ff}$.

${ }^{25}$ Waldenfels kondensiert Levinas' Konzept auf die prägnante Formel „The human being is a being which has a face“. Waldenfels, Bernhard: Levinas and the face of the other. In: Simon Critchley/Robert Bernasconi (Hg.): The Cambridge Companion to Levinas. Cambridge 2002, S. 63-81, hier S. $67 \mathrm{f}$.

${ }^{26}$ Vgl. auch Stegmaier: Emmanuel Levinas, S. $134 \mathrm{ff}$.

${ }^{27}$ Denn das Gesicht ist ,the corporeal emblem of the other's otherness“. Waldenfels: Levinas and the face of the other, S. 63.

${ }^{28} \mathrm{Vgl}$. Waldenfels: Levinas and the face of the other, S. 70.
} 
verdeckt. „Death had done its work“ (101), ,he“ wird zum gesichtslosen ,it" und damit (m)essbar. Du Chaillu ,had leisure“ (101), den gerade noch schreckenserregenden Körper zu untersuchen, zu vermessen und damit in einen Wissenschaftsgestus aufzunehmen, während seine Männer, ,though rejoicing at our luck“, sich bereits um Anteile am Gorilla-Fleisch streiten, „for they really eat this creature" (101, Hervorheb. MS). ${ }^{29}$ Du Chaillus affektive Inszenierung der Gorillajagd und seines sentimentalen (Selbst-)Erlebens läuft derart auf die Tötung des Gorillas zu, die gleichzeitig den zuvor zum ultimativen Gegner anthropomorphisierten Affen in seiner Bedrohung für Du Chaillus Selbstverständnis antiklimaktisch re-animalisiert.

\title{
2.1.3 Der nüchterne Sammler Alfred Russel Wallace
}

Lässt sich Paul Du Chaillus Gefühlslage in ihrer Intensität und zeitweiligen Ambivalenz an dem selbstverliehenen Doppelbegriff des ,naturalist-hunter“ $(E A, 268)$ festmachen, so ist Alfred Russel Wallace der entomologisch fokussierte Naturkundler. Die Jagd ist ihm kein Vergnügen an sich; er lässt lieber schießen, als dies selbst zu tun: „During my stay in Borneo I had no hunter to shoot for me regularly, and, being myself fully occupied with insects, I did not succeed in obtaining a very good collection of the birds or Mammalia“ (MA, 61), so räumt er ein. Die erste, zufällige Begegnung mit einem Orang-Utan weist daher bei Wallace auch nicht das affektive Repertoire des kulturellen Modells Jagd auf: ${ }^{30}$

\begin{abstract}
Just a week after my arrival at the mines, I first saw a Mias. I was out collecting insects, not more than a quarter of a mile from the house, when I heard a rustling in a tree near, and, looking up, saw a large red-haired animal moving slowly along, hanging from the branches by its arms. This mode of progression was, however, very unusual, and is more characteristic of the Hylobates, than of the Orang. I suppose there was some individual pecularity in this animal, or the nature of the trees just in this place rendered it the most easy mode of progression. (62)
\end{abstract}

Die Fauna Borneos ist für den inselbiogeografisch denkenden Wallace ,peculiar“, in diesem Orang-Utan sogar von ,individual percularity“.31 Doch es ist bemerkenswert, wie wenig affektiv Wallace diese erste Sichtung des „chief

\footnotetext{
${ }^{29}$ Richter verweist denn auch darauf, dass es genau diese durch den Fast-Kannibalismus markierte „ambiguity of colonial desire“ der erlaubten Transgression ist, die für Du Chaillu die Gorilla-Jagd zur „ultimate challenge“ werden lässt. Richter: Literature after Darwin, S. 34 f.

${ }^{30}$ Zum Modellcharakter der Jagd vgl. Borgards, Roland: Tiere Jagen. Gasteditorial. In: TIERethik 5/7 (2013), S. 7-11.

${ }^{31} \mathrm{Zu}$ Wallaces Beschäftigung mit dem ,faunistischen Element ' von Inseln, die 1880 in die Publikation Island Life münden sollte, siehe das erste Kapitel („Physical Geography“) des Malay Archipelago, S. 1-30 sowie Wallace, Alfred Russel: Island Life; or, The Phenomena and Causes of Insular Fauna. London 1880.
} 
object[]“ (61) seiner Reise ausgestaltet. Hier zeigt sich keine Freude, keine Anspannung oder Aufregung, stattdessen wird nüchtern notiert, wie sich das Tier bewegt, und diese „mode of progression“ abgeglichen mit dem bestehenden Wissen über die Fortbewegung der Spezies. Zwei Wochen später greift Wallace zwar gleich zur Waffe, als er hört, dass ein weiterer Orang-Utan in der Nähe frisst. Doch hier heißt es nur knapp: „As soon as I approached, it tried to conceal itself among the foliage; but I got a shot at it, and the second barrel caused it to fall down almost dead, the two balls having entered the body. This was a male, about half-grown, being scarcely three feet hight" (62). Diese Passage ist weniger Szene als Auflistung. Einer Auflistung gleicht auch Wallace’ weiterer Bericht über seine Sichtungen von Orang-Utans, die er nun ungefähr im Wochentakt von den Bäumen schießt:

On April 26th, I was out shooting with two Dyaks, when we found another about the same size. It fell at the first shot, but did not seem much hurt, and immediately climbed up the nearest tree, when I fired, and it again fell, with a broken arm and a wound in the body. The two Dyaks now ran up to it, and each seized hold of a hand, telling me to cut a pole, and they would secure it. But although one arm was broken and it was only a half-grown animal, it was too strong for these young savages, drawing them up towards its mouth notwithstanding all their efforts, so that they were again obliged to leave go, or they would have been seriously bitten. It now began climbing up the tree again; and, to avoid trouble, I shot it through the heart. (62 f.)

On May 2d, I again found one on a very high tree, when I had only a small 80-bore gun with me. However, I fired at it, and on seeing me it began howling in a strange voice like a cough, and seemed in a great rage, breaking off branches with its hands and throwing them down, and soon made off over the tree-tops. I did not care to follow it, as it was swampy, and in parts dangerous, and I might easily have lost myself in the eagerness of pursuit.

On the 12th of May I found another, which behaved in a very similar manner, howling and hooting with rage, and throwing down branches. I shot at it five times, and it remained dead on the top of the tree. [...] I therefore returned home, and luckily found some Dyaks, who came back with me, and climbed up the tree for the animal. This was the first fullgrown specimen I had obtained; but it was a female, and not nearly so large or remarkable as the full-grown males. [...] I preserved the skin of this specimen in a cask of arrack, and prepared a perfect skeleton, which was afterwards purchased for the Derby Museum. (63 f.)

Im Gegensatz zu Du Chaillus Explorations and Adventures erscheint Wallace' Text frei von Empathie für die Tiere. Er jagd und schießt, bzw. lässt jagen und schießen, um sie qua taxidermischer Präparation in eine Sammlung einzureihen. Wohl schreibt er den Affen emotionale Zustände zu (,seemed in a great rage“, „howling and hooting with rage“, 63) und versieht sie mit einem typischen Verhaltensrepertoire (das Zweigebrechen und -werfen). Er selbst enthält sich jedoch in seinem Text einer Selbstbeobachtung affektiver Valenz. Es geht ihm darum, „to avoid trouble“ (63), oder sich selbst nicht in der „eagerness of pursuit“ (63) in gefährlichem Sumpfgebiet zu verirren. Ist die Verfolgung der Affen bei Du Chaillu mit einer starken körperlichen Empfindung verbunden - dem Rausch (,rush“), den er mehrfach erlebt (z. B. EA, 86) - überwiegt bei Wallace eine intellektuelle Haltung: „eagerness“. Dies entspricht einem Vokabular des wissenschaftlichen 
Interesses und der Faszination des Sammlers, der sich, wenn überhaupt, dann um die Durchführbarkeit und den Erfolg seines Vorhabens sorgt. Selbst die darin involvierten Aufmerksamkeitsphänomene - Interesse und Faszination - werden nicht explizit dargestellt. ${ }^{32}$ Dies mag damit zusammenhängen, dass er zunächst nicht jenes wahre ,chief object' einer naturkundlichen Sammlung erlangt. Denn „,it was a female“ (64), und in der Ordnung der Dinge, der Wallace und nach ihm Naturforscher bis in das letzte Drittel des 20. Jahrhunderts hinein folgen, ist allein das Männchen sowohl Krönung der naturhistorischen Sammel- und Jagdleidenschaft als auch einziger adäquater Repräsentant seiner Art. ${ }^{33}$ Deutlich zeigt sich dies daran, dass Wallace die Textpassage, welche von der ersten Begegnung mit einem ausgewachsenen Orang-Utan-Männchen handelt, auch wesentlich szenischer auf den Effekt und die Klimax hin gestaltet: Von einer entomologischen Exkursion zurückgekehrt,

Charles [Wallace' Assistent] rushed in out of breath with running and excitement and exclaimed, interrupted by gasps, ,Get the gun, sir; - be quick, - such a large Mias!‘ ,Where is it?' I asked, taking hold of my gun as I spoke which happened luckily to have one barrel loaded with ball. ,Close by, sir - on the path to the mines - he can't get away“. (72)

Die in The Malay Archipelago seltene wörtliche Rede potenziert die Aufregung und ihre physischen Erregungssymptome außergewöhnlich. Sie werden auf den Feldassistenten Charles projiziert, an dem sich die Anzeichen des emotionalen Aufruhrs objektivierend beschreiben lassen (,,rushed in out of breath with running and excitement and exclaimed, interrupted by gasps“"). Nach diesem markiert anderen Auftakt wechselt Wallace zum Jagdnarrativ:

\footnotetext{
We walked cautiously along, not making the least noise, and listening attentively for any sound which might betray the presence of the Mias, stopping at intervals to gaze upwards. $[\ldots][\mathrm{W}] \mathrm{e}$ dispersed a little, feeling sure that it must be somewhere near [...]. Very soon, however, one of the Dyaks called me and pointed upwards, and on looking I saw a great hairy body and a huge black face gazing down from a great height, as if wanting to know what was making such a disturbance below. I instantly fired, and he made off at once, so that I could not then tell whether I had hit him. (74)
}

\footnotetext{
${ }^{32}$ Zur Mehrdeutigkeit und Mehrwertigkeit der Faszination als (auch ästhetische) Emotion siehe Lüdtke, Jana/Jäkel, Anne/Ordonez Acuna, Daniela: Self reported fascination experiences. Approaches to an unexplored emotion. In: Martin Baisch/Andreas Degen/Jana Lüdtke (Hg.): Wie gebannt. Ästhetische Verfahren der affektiven Bindung von Aufmerksamkeit. Freiburg u. a. 2013, S. 303-332; Degen, Andreas: Concepts of Fascination, from Democritus to Kant. In: Journal of the History of Ideas 73/3 (2012), S. 371-393. Zum Interesse als alle Forschung erst aktivierendem Basisgefühl wiederum Ciompi, Luc: Die emotionalen Grundlagen des Denkens. Entwurf einer fraktalen Affektlogik. Göttingen 1997, S. 121, 201-208.

${ }^{33}$ Vgl. Haraway: Primate Visions, S. 41. Zu den komplexen Zusammenhängen von Leithypothesen und Geschlechterpräferenz in der Geschichte der Primatologie siehe Strum/Fedigan: Changing Views of Primate Society; Fedigan: Primate Paradigms, S. xxxv-xxxviii.
} 
Nicht nur wird hier die Anspannung der Jäger adverbial (,,cautiously“, ,attentively“, „instantly“) und deskriptiv (,not making the least noise“) erstmals in den Blick genommen. Das Desiderat-Tier tritt auch anders als zuvor als ein geschlechtliches Individuum, als „,he“, zutage und lohnt der einfühlenden Spekulation: ,[A]s if wanting to know", schreibt Wallace, um dem Blick des Orang-Utan hier eine Bedeutung zu verleihen, die dem szenischen Narrativ seiner Jagd einen Sinn gibt. Es ist dieser männliche Orang-Utan, der Wallace zum ersten Mal zu einer direkten Gefühlsäußerung verleitet:

The Mias did not move, and I began to fear that after all we should not get him [...]. As a last resource we all began pulling at the creepers [...], and after a few minutes, when we had almost given up all hopes, down he came with a crash and a thud like the fall of a giant (75, Hervorheb. MS).

In diesem letzten Satz steckt einerseits der objektivierende Vergleich des Tieres mit einem gigantischen, gefällten Baum (,with a crash and a thud like the fall of a giant"), andererseits ist dieser Orang-Utan für Wallace, der erstmals durch das Tier affiziert scheint, nachdrücklich eine aufs Mythische hin gedachte Figur - in ihrer Menschenähnlichkeit: „And he was a giant, his head and body being full as large as a man's“ (75). Dieses besondere, das legendäre Tier, der Riese des Urwalds, bietet bereits den emotionalen Höhepunkt von Wallace' Orang-Utan-Passagen aus The Malay Archipelago. Bald danach verkommt das Sammeln von Orang-Utans zu einer Gewohnheit, die Wallace emotional höchstens im Spektrum des Alltagsärgernisses erlebt. Teilweise tendiert die emotionslose Beschreibung zur pathologischen Kaltblütigkeit: Die Tiere werden immer geschossen, auch wenn man die Körper gar nicht zu erlangen hoffen kann, und Wallace keinen Genuss aus der Jagd als Tätigkeit zieht. Dass ein Körper in den Baumwipfeln zurückgelassen werden muss, ist bedauerlich, jedoch wird das Bedauern nur explizit erwähnt (,This I regretted much“, 77), wenn es sich um Männchen handelt. ${ }^{34}$ Die relative affektive Gleichgültigkeit im Umgang mit dem tierlichen Wesen und dem Affenkörper ist Teil einer Arbeitsroutine, die Wallace wie folgend beschreibt:

I cut off the head and took it home to clean, while I got my men to make a close fence about five feet high round the rest of the body, which would soon be devoured by maggots, small lizards and ants, leaving me the skeleton. (77)

As it was too far to carry the animal home, I set to work and skinned the body on the spot, leaving the head, hands and feet attached to be finished at home. (84)

Die Elaboration in der Schilderung der Präparation dieser je nach Lesart Jagdoder Forschungs-Trophäen legt einen Stolz auf das eigene Fachwissen und Können nahe. Sie ist jedoch weit entfernt von der Selbstinszenierung als empfindsamer

\footnotetext{
${ }^{34}$ Wallace bedauert, nicht an den Körper zu kommen, ,as it was a fine full-grown male“ (MA, S. 77). Demgegenüber interessieren ihn die andere Exemplare nur wenig: „And as young animals are of comparatively little interest, I did not have the tree cut down to get it“" (S. 81).
} 
Jäger, wie sie Du Chaillu praktiziert. Nur ein einziges Mal scheint Wallace durch die Tätigkeit, die er hier verübt, in der Selbstrepräsentation als nüchterner Naturforscher unterminiert. Er erwischt einen ,very old male Mias“, den er jedoch in einem Sumpfgebiet erlegen muss, ,and after several shots under these trying circumstances, I was delighted to see the monstrous animal roll over into the water" (88, Hervorheb. MS). Dies ist das einzige Mal, dass Wallace das Adjektiv „monstrous“ auf einen der Affen anwendet, und eine seltene Instanz, in der er Erleichterung bzw. Freude ob des gelungenen Schusses bekundet. Dies wird der letzte Orang-Utan sein, den Wallace in The Malay Archipelago schießt. Somit bewegt sich Wallace' Schilderung der Affen-Sammlung in diesem Rückgriff auf den Monster-Topos des Affen dramatisch ebenfalls auf einen - besonders in der Erwähnung der Erleichterung - kathartischen Abschluss zu.

\subsubsection{Emotionale Wende am Affenbaby}

Besteht sowohl bei Alfred Russel Wallace als auch bei Paul Du Chaillu die Beschäftigung mit dem Affen hauptsächlich aus der Jagd und Sammlung, finden sich doch in beiden Berichten auch andere Schlüsselpassagen für affeninduzierte Affekte: der Fund von lebenden Jungtieren. Ist das ausgewachsene Männchen der optimale Endgegner im Spiel der Jagd (Du Chaillu) und das Prachtexemplar einer jeden naturkundlichen Sammlung (Wallace), so bietet das Affenjunge die Möglichkeit, das angeborene Verhalten der Tiergattung zu studieren und zugleich ein eigenes artrelevantes emotionales Reservoir anzuzapfen, das durch ein primatenübergreifendes Kindchen-Schema aktivierbar ist. ${ }^{35}$ Auch hier zeigt sich zunächst die konträre emotionale Färbung der Texte deutlich: Für Du Chaillu ist der Fund des Gorillababys „one of the greatest pleasures of my whole life“ (EA, 242): „Some of the hunters who had been out on my account brought a young gorilla alive!" (242, Hervorheb. im Original), schreibt er und versieht die Textpassage gleich zu Beginn mit einem Stich des kleinen Gorillas (242). Wieder ist er um eine Wiedergabe seiner Empfindungen bemüht: „I can not describe the emotions with which I saw the struggling little brute dragged into the village. All the hardships I had endured in Africa were rewarded in that moment" (242 f.). Wie-

\footnotetext{
${ }^{35}$ Das zunächst 1943 von Konrad Lorenz theoretisierte instinktauslösende Kindchen-Schema wird auch von den Neurowissenschaften in Hinblick auf Emotionsaktivierung, Interdependenzen, Spezifizität und Universalität untersucht. Vgl. Lorenz, Konrad: Die angeborenen Formen möglicher Erfahrung. In: Zeitschrift für Tierpsychologie 5 (1943), S. 233-519; Brosch, Tobias/Sander, David/Scherer, Klaus R.: That Baby Caught My Eye... Attention Capture by Infant Faces. In: Emotion 7/3 (2007), S. 685-689; Lehmann, Vicky/Huis in't Veld, Elisabeth M. J./Vingerhoets, Ad J. J. M.: The human and animal baby schema effect: Correlates of individual differences. In: Behavioural Processes 94 (2013), S. 99-108; Sato, Anna u. a.: Visual Recognition of Age Class and Preference for Infantile Features: Implications for Species-Specific vs Universal Cognitive Traits in Primates. In: PLoS ONE 7/5 (2012), S. e38387.
} 
der erscheint dieses emotionale Erleben im Detail unbeschreiblich, und wird doch beschrieben durch ein bekanntes Repertoire an emotionalen Phrasen: ,the hardships $[\ldots]$ rewarded“, ,,[m]y hunters, whom I could have hugged to my heart, [...] determined to risk all, [....] to take the young one alive, knowing what a joy it would be to me" (243).

Wallace dagegen findet unter einem der geschossenen Weibchen schlicht ,a young one face downwards in the bog" (MA, 65) und gibt wieder keine Auskunft über seine emotionale Wahrnehmung des Ereignisses, sondern zieht sich auf eine beschreibende Perspektive zurück: „This little creature was only about a foot long, [...]. Luckily it did not appear to have been wounded, [...] and seemed quite strong and active“ (65). Selbst als das Orang-Utan-Baby sich auf dem Weg zurück ins Lager an seinem Bart festklammert, stellt Wallace nur fest: ,[I] $\mathrm{t}$ got its hand in my beard, and grasped so tightly that I had great difficulty in getting free, for the fingers are habitually bent inwards at the last joint so as to form complete hooks“ (65). Jede Regung des Tierjungen wird registriert und als arttypisches Verhalten notiert. Das registrierende „I“ lässt jedoch keinerlei Einlassung auf eine emotionale Wahrnehmung zu. Dies zeigt sich prägnant in Formulierungen wie „I was therefore obliged“ (65) (das Orang-Utan-Baby mit einer Flasche zu füttern), „I soon found it necessary“ (66) (das Junge zu säubern), und sehr deutlich in „I was obliged to take the imitation mother to pieces again“ (68). Das kleine Jungtier, so geht aus Wallace' Schilderung hervor, greift sich ihn als Bezugsperson, will von ihm gewaschen, umhegt und gepflegt werden, zeigt Symptome des Hospitalismus, wenn es sich immer wieder selbst am Fell zieht, braucht schließlich eine Mutter-Attrappe aus Fell, die es überstrapaziert. Auch das infantile Tier wird in Wallace' Erzählung auf emotionaler Distanz gehalten. Im Laufe seiner Schilderung zeigt sich jedoch die Wirkmacht des kindlichen Charmes auch in der Begegnung von Forschersubjekt und Affenjunges: Das Tier bleibt für ihn zwar ein ,it“ und meist „the little Mias“, wird jedoch von ,a young one“ (65) über ,,[t]his little creature“ (65) zu „,my little infant“ (68), von ,like a baby“ (68) zu ,exactly like a baby“ (69), von „,the little orphan“ (68) zu ,the poor little thing“(68). Das Affenbaby macht „ridiculously wry faces“(66), genießt das Trockenreiben ,amazingly“ (66) und „seemed to be perfectly happy“ (66), wenn Wallace es kämmt. Es hält sich zunächst „desperately“ (66) an allem fest, ,seemed quite happy“ (67), wenn es etwas zu greifen bekommt. Die Mutterattrappe „seemed to suit it admirably“ (68), sodass Wallace konstatiert: „I was now in hopes that I had made the little orphan quite happy“ (68). Es ist ihm sogar ,a never-failing amusement to observe the curious changes of countenance“ (68), mit denen der kleine Affe seine geschmacklichen Erlebnisse nach außen trägt. Das hier am Affenkind aktivierte Einfühlungspotenzial kann besonders dort gezeigt werden, wo Wallace dem Orang-Utan einen ,young hare-lip monkey“ (69) als lebendes Spielzeug beigibt: 
The little monkey would sit upon the other's stomach, or even on its face, without the least regard to its feelings. [...] The little helpless Mias would submit to all these insults with the most examplary patience, only too glad to have something warm near it, which it could clasp affectionately in its arms (69, Hervorheb. MS). ${ }^{36}$

Die Erfahrung der Empathie scheint insofern transformierend zu sein, als sie Wallace eine der seltenen emotionalen Selbstauskünfte abringt, als der kleine Orang stirbt:

I much regretted the loss of my little pet, which I had at one time looked forwards to bringing up to years of maturity, and taking home to England. For several months it had afforded me daily amusement by its curious ways and the inimitably ludicrous expressions of its little countenance. (72)

Hier zeigt sich aber zugleich auch Wallace' Umgang mit der emotionalen Transgression, die der kleine Orang-Utan in diesen Borneo-Aufenthalt getragen hat: Obwohl als bedauerlicher Verlust (,loss“) empfunden, macht der Tod des kleinen Orang ihn wieder geeignet für eine Einordnung in die hierarchische Taxonomie, in der das Tier nur ,pet“ und ,possession“ des Menschen sein kann: „It lost all its appetite for its food, and after lingering for a week a most pitiable object, died, after being in my possession nearly three months“ (71, Hervorheb. MS). Konsequenterweise informiert Wallace seine Leser/innen: „I preserved its skin and skeleton“(72).

Auch Du Chaillus Gorillajunges wird schließlich sterben, doch zuvor hat der „young male gorilla“ (EA, 244) in Du Chaillus Bericht einigen Raum zur Verfügung, um sich als Figur eines Narrativs zu entfalten. Du Chaillu erzählt auf fast drei Seiten von seiner Episode mit dem Affenbaby, das wie ein gefangener Sklave mit seinem Hals in einem „forked stick“ (244) ins Dorf gebracht wird. Das Tierjunge ist für ihn „the little brute, [...] the merest baby for age“ (244), „this little beast" (245), „my captive“ (245), „the little rascal“" (245) und „the little fellow“ (245). Er nennt den kleinen Gorilla konsequent „he“ und gibt ihm den Namen Joe (245). Du Chaillus Erzählung beschreibt ein Interagieren zwischen Joe und Du Chaillu, in dem der Gorilla als Problemkind figuriert. ,[A]stonishingly strong, and by no means good-tempered“ (244), muss er in einen Käfig gesperrt werden, reißt Du Chaillus Hosenbein auf, bricht aus dem Käfig aus, und benimmt sich auch ansonsten wenig dankbar für seine ,Rettung`. Sein Charakter wird stimmig zu dem

\footnotetext{
${ }^{36}$ Auch in einem Brief vom 25. Juni 1855 an seine Schwester Frances lässt sich ein solcher zum Gefühl hinarbeitender Verlauf des Berichtens nachvollziehen. Ist Wallace darin auch zunächst bemüht, davon zu berichten, wie er das Affenbaby erlangt und versorgt hat, also seine Fertigkeiten hervorzuheben, so endet die Passage schließlich mit einem Absatz zur rührenden Einzigartigkeit des Babys: „From this short account you will see that my baby is no common baby, and I may safely say, what so many have said before with much less truth, ,There never was such a baby as my baby " - and I am sure nobody ever had such a dear little duck of a darling of a little brown hairy baby before!“. van Wyhe, John/Rookmaaker, Kees (Hg.): Alfred Russel Wallace. Letters from The Malay Archipelago. Oxford 2013, S. 49, Hervorheb. im Original.
} 
des erwachsenen Gorillas gezeichnet, er ist ebenfalls ,furious, [...] with his eyes glaring and every sign of rage in his little face and body“, angriffslustig, „morose and savage" (245). ${ }^{37}$ Doch bei diesem Jungtier empfindet Du Chaillu den widerspenstigen und kämpferischen Charakter als ,a temper thoroughly wicked and malicious“ (246). So kommt er zu dem Schluss: „I never saw so furious a beast in my life as he was“"(245).

Diese Empathie hat bei Du Chaillu einen narrativen Zweck: Joe wird hier als Tier wahrgenommen und zugleich mit einer Deutung emotionaler Zustände und einer behavioralen Beschreibungen versehen, die ihn zur lebhaften Figur in einem pädagogischen Narrativ machen. Du Chaillu selbst tritt in seiner Emotionalität in dieser Passage dagegen zurück - im Vergleich zu der deutlichen Freude und Begeisterung, die er zu Beginn über den Babyfund äußert, hat er nun hauptsächlich Angst um die Besitztümer in seiner Hütte (vgl. 246). Im Verlauf dieser Passage zeigt sich durch die Schilderung der zähmenden und beschwichtigenden Bemühungen Überdruss an der Wildheit und dem Freiheitsbedürfnis des Tieres. Es scheint fast so, als sei der kleine Gorilla in erster Linie „most troublesome“ (246) gewesen, sodass sein Tod auch mitten in der Erzählung symbolisch bereits vorweggenommen wird. ${ }^{38}$ Der tatsächliche Tod wird dann mit einer Art Nachruf bedacht - „To the last he continued utterly untamable“ (247) - aus der sich eine emotionale Schilderung von $\mathrm{Du}$ Chaillus Erleben zumindest explizit nicht erschließt.

Am Affenkind kehren sich also die affektiven Rollen der beiden Forschungsreisenden um: Der ,hunter-naturalist ${ }^{\star}$ Du Chaillu, der die Rolle des Jägers mit einem affektiv besetzten Verhältnis zum Beutetier ausfüllt und sein emotionales Erleben narrativ ausschöpft, reduziert gerade dort seine Empfindsamkeit und Einfühlung, wo diese über die Formung einer antagonistischen Figur in einer Erzählung hinausgehend Sympathie für die verwaiste Kreatur fordern würde. Das Gorillababy ist hier jedoch vor allem die herausfordernde Miniatur des Gorillas als Endgegner des weißen Jägers. Die kämpferischen und trotzenden Charakterzüge, die im ausgewachsenen Tier die Eignung zum begehrten und angsteinflößenden Monster belegen (,fierce“, „furious“, „rage“), sind im Kleinen lästig und unliebsam. Die Verweigerung, gezähmtes Haustier zu werden, wird von Du Chaillu als negativer Charakterbeweis und in gewisser Weise auch als

\footnotetext{
${ }^{37}$ Ebenso wie der Gorilla im Vorwort wird auch ,Master Joe“ mit einem zahmen Affen kontrastiert, der all das erfüllt, was der Gorilla missen lässt: der ,nshiego “ „Master Tommy“ ( $E A$, S. 329-333). In dessen „,sentimentale[r] Präsentation“ sieht Julika Griem deutlich Du Chaillus Identifikation mit diesem ,weißen“ Affen (im Gegensatz zu dem ,schwarzen“ Gorilla) aufscheinen, die zugleich eine imperialistische Hierarchie bestätigt, verlagert und subervertiert. So wenn du Chaillu seine Träger einen Vergleich zwischen ihm und dem weißgesichtigen jungen Affen anstellen lässt: „See white face of your cousin from the bush! He is nearer to you than gorilla is to us" (EA, S. 330). Griem: Monkey Business, S. 86.

${ }^{38}$ Vgl. EA, S. 247. Bereits zu Beginn, wenn es heißt, der Käfig sei fertig und „,[h]ere the thing was immediately deposited; and now [...] I had a fair chance to look at my prize“ (ebd., S. 244), wird der kleine Gorilla breits zur leblosen Trophäe im Schaukasten.
} 
persönliche Beleidigung betrachtet. So wimmelt diese Passage von Vokabeln moralischer Zweideutigkeit oder sogar Verwerflichkeit: Nicht nur ist der kleine Gorilla „wicked and malicious“ (246), er hat auch ,venomous and sullen eyes“ (246), ist voll ,treachery“ (247) und hegt ,,a feeling of revenge“ (246). Im Gegenzug wird das Affenjunges nicht nur im Käfig eingesperrt, es wird auch sukzessive ausgehungert und in Ketten gelegt, bis es stirbt. Alfred Russel Wallace dagegen, dessen gesamter Bericht von der emotionalen Distanziertheit zeugt, mit der jener Forscher seinen Forschungsobjekten gegenübertritt, der für die akribische Suche nach Insekten mehr Leidenschaft aufzubringen scheint als für den Jagdsport, und dessen Auflistung der erlegten Spezimina in ihrer Emotionslosigkeit an der Grenze des Pathologischen angesiedelt sein mag, ausgerechnet Wallace erwärmt sich für das Orang-Utan-Baby, das ihm in die Hände fällt. Wallace' Schilderung des Orang-Utans erscheint im Rahmen seines Textes auffällig affektiv besorgt. Auch wenn Wallace dem Affenbaby keinen Namen gibt oder zumindest seinem Publikum keinen mitteilt, ${ }^{39}$ zeugt die Passage ebenso wie das seltene Selbstbekenntnis zum emotionalen Verlust von der Zuneigung und Sorge, die Wallace für den fordernden kleinen Orang-Utan zumindest wie zu einem Haustier empfunden haben mag. Für den Einen also ist der lebendige kleine Affe die Ausnahme, die ein emotionales Erleben mittels einer die Wahrnehmung und den Bericht bereichernde Empathie ermöglicht. Für den Anderen werden am lebenden Exemplar die Grenzen einer Inszenierung als empfindsames Subjekt deutlich, das empathisch nur im Sinne seiner eigenen Narrativierung auftritt, in der Tiere im Rahmen der Jagd als Instrumente der sentimentalen Empfindsamkeit genutzt werden.

\subsubsection{Symptomatisches Fühlen}

Die von Wallace und $\mathrm{Du}$ Chaillu repräsentierten Forscherpersönlichkeiten, Empfindungsmuster und Narrative sind Modelle, mit denen sich spätere primatologische Feldforscher/innen in ihren Berichten und in ihrer Selbstrepräsentation auseinandersetzen müssen, wenn sie sich selbst zwischen den Polen schwärmerischen emotionalen Erlebens einerseits und kühler Distanziertheit des wissenschaftlichen Berichts andererseits verorten. Darüber hinaus zeigen diese beiden Texte Schlüsselszenen der affektiven Affenbegegnung und zeugen von einer Methodik der Naturforschung, die potenziell ein zugleich emotionalisierendes und distanzierendes Verhältnis zum Tier in sich birgt. Wesentlich dafür ist die Jagd als ein kulturelles und historisches Modell der Annäherung an und Einfühlung in das Tier, aber auch der distanzierenden Abwehr emotionaler

\footnotetext{
${ }^{39} \mathrm{Im}$ Brief an die Schwester lässt Wallace allerdings vermehrt den Artikel wegfallen, sodass ,baby' zum Eigennamen des Orang-Utans wird: „When the monkey wants to run away [...], baby clutches him by the tail [...]. Of course, baby cannot walk yet“. van Wyhe/Rookmaaker (Hg.): Letters, S. 49.
} 
Identifikation mit dem Anderen. ${ }^{40}$ Diese ohnehin schon problematische Ambivalenz der Jagd wird durch den Affen und seine Menschenähnlichkeit potenziert. Einerseits ist der Menschenaffe der ,prize at which I had been hoping for years to get a shot" $(E A, 85)$, die Trophäe, welche die Autorität „,der imperialistischen Schlüsselfigur des weißen Jägers" 41 sichert. Andererseits subvertiert die physische und behaviorale Ähnlichkeit der großen Affen diese Selbstbestätigung im Lustgewinn und Prestige der Jagd gerade durch ihre Schlüsselfunktion für das emotionale Reservoir. „There is enough likeness to humanity in this beast“, schreibt Du Chaillu gegen Ende seiner Explorations and Adventures, „to make a dead one an awful sight, even to accustomed eyes, as mine were by this time. I never quite felt that matter-of-course indifference, or that sensation of triumph which the hunter has when a good shot has brought him a head of his choice game“ (487). Die Affekte, die selbst (oder gerade) der getötete Affe auslöst, zeichnen das Forschersubjekt als empfindsamen Reisenden aus. Sie sind hier jedoch nicht mehr wie jene der Aufregung und Anspannung willkommen, sondern werden in ihrer Intensität und ihrer Überwältigung des Subjektes als Fehler im Ablauf des Jagd-, Forschungs- und Reiseprozesses wahrgenommen, der darauf verweist, dass hier etwas nicht stimmt: „It was as though I had killed some monstrous creation, which yet had something of humanity in it. Well as I knew this was an error, I could not help the feeling" (487, Hervorheb. MS). Es lässt sich fragen, inwiefern diese emotionale Überwältigung - die nicht zuletzt auf einem kulturell geprägten moralischen Empfinden beruht, das die Welt einteilt in Tötbares (nichtmenschliche Tiere) und Untötbares (menschliche Tiere, mit Einschränkungen) - jedoch nicht ein Fehler im System sondern ein systematisches Symptom ist. Donna Haraway hat bereits darauf hingewiesen, dass die Jagd als wesentlicher Bestandteil der Naturforschung bis zur Mitte des 20. Jahrhunderts einhergeht mit optischen Medien der Distanzierung (dem Zielrohr des Gewehres, dem Feldstecher), die als Instrumentarium zwischen das beobachtende und fühlende Subjekt und das Objekt seiner Beobachtung gesetzt werden. Dies setzt sich für Haraway auch noch fort im Kamerasucher, der z. B. bei Carl Akeley zur Anwendung kommt und Distanz schafft, bevor das Tier dann doch auch wieder geschossen wird. ${ }^{42}$ Lange Zeit bestimmte eine solche ,mediale Distanzierung die Forschung am Tier, in der zudem Aufschluss über das Tier nur aus dem Tierkadaver, oder aber über gefangene und aus einem ,natürlichen Raum' entnommene Jungtiere entstand. Der Beginn der ethologischen Feldforschung an (Menschen-)Affen revolutioniert nicht nur diese Form der empirischen Forschung, sondern ermöglicht, indem sie weitgehend auf das Distanzierungsinstrumentarium verzichtet und gerade die räumliche Distanz in der Beobachtung zu minimieren versucht, auch eine neue oder bislang verhinderte Form des potenziellen emotionalen Erlebens und Wahrnehmens. Die langan-

\footnotetext{
${ }^{40} \mathrm{Vgl}$. Borgards: Tiere jagen.

${ }^{41}$ Griem: Monkey Business, S. 86.

${ }^{42}$ Vgl. Haraway: Primate Visions, S. $41 \mathrm{ff}$.
} 
haltende Erfahrung räumlicher Nähe zum lebenden Tier ermöglicht, wie die folgenden Kapitel ausführen, Gefühle in einem ,Drama of Touch', das ,Gefühl als Getast', die Emotion als Erkundung und Erkenntnismöglichkeit.

\subsection{Dramaturgien des taktilen Erstkontakts}

I made it through the wilderness

Somehow I made it through

Didn't know how lost I was

Until I found you

I was beat incomplete

I'd been had, I was sad and blue

But you made me feel

Yeah, you made me feel

Shiny and new

Like a virgin

Touched for the very first time

Like a virgin

When your heart beats next to mine

Gonna give you all my love boy

My fear is fading fast

Been saving it all for you

'Cause only love can last ${ }^{43}$

\subsubsection{Das ,Drama of Touch' der Primatologie}

Die Britin Jane Goodall, die US-Amerikanerin Dian Fossey und die Kanadierin Biruté Galdikas greifen tradierte Elemente des Reise- und Forschungsberichts bereitwillig auf, um ihr Publikum an die Forschungsarbeit heranzuführen. Ihre Motivation für die Forschung mit Affen ,im Feld' wird ebenso erörtert wie die Anreise in das jeweilige Habitat und seine Eigenartigkeit als ,fremdes Land ‘ ${ }^{44}$ Was sie jedoch von früheren primatologischen Texten, die Berichte über kurze Feldaufenthalte beinhalten, und den im vorangegangenen Kapitel diskutierten Reisetexten unterscheidet, ist Folgendes: Sie privilegieren wissenschaftlich methodisch und textuell narrativ die einfühlende Beobachtung und die Nähe zum Beobachteten. Die Berührung zwischen Mensch und Tier nimmt hier eine zentrale Rolle ein als Signifikant einer Veränderung in der Beziehung zwischen

\footnotetext{
${ }^{43}$ Madonna: Like a Virgin. Sire/Warner Brothers Records 1984.

${ }^{44} \mathrm{Vgl}$. bspw. van Lawick-Goodall, Jane: In The Shadow of Man, S. 1-12; Fossey, Dian: Gorillas in the Mist [1983]. London 2001, S. 1-7; Galdikas, Biruté M. F.: Reflections of Eden: My Years with the Orangutans of Borneo. Boston u. a. 1995, S. 19-45, 67-91. Im Folgenden, wenn zur Unterscheidung nötig, als $S M, G M$ und $R E$ geführt; Seitenangaben im Text.
} 
beobachtender Forscherin und beobachtetem Tier. Zugleich ist sie Teil einer epistemischen Semantik, die Erkenntnis qua Emotion zulässt. Wie im Folgenden gezeigt wird, arbeiten die drei populärwissenschaftlichen Kerntexte dieses Neubeginns der Primatologie in den 1960er und 1970er Jahren - Goodalls In the Shadow of Man (1971), Fosseys Gorillas in the Mist (1983) und Galdikas' Reflections of Eden (1995) - die Bedeutung emotionaler Erkenntniswerte dramaturgisch in Szenen taktiler Begegnung heraus. ${ }^{45}$

Bei allen drei Autorinnen sind es einzelne Affen, die in isolierten Szenen der körperlichen Berührung zu Akteur/innen eines epistemologischen, auf physischer und emotionaler Nähe basierenden Austausches werden. Bei Goodall ist dies David Graybeard, wie die Forscherin eines der Schimpansenmännchen wegen seines grauen Gesichtsfells nennt. ${ }^{46}$ David Graybeard beschert Goodall einige besondere Fortschritte in ihrem Langzeitforschungsprojekt, so z. B. den ersten Blickkontakt mit dem Untersuchungstier. Dieser Blickkontakt beendet eine frustrierende Periode zu Beginn von Goodalls Aufenthalt in Gombe, in der sie kaum jemals einen Affen beobachten konnte:

Less than twenty yards away from me two male chimpanzees were sitting on the ground staring at me intently. Scarcely breathing, I waited for the sudden panic-stricken flight that normally followed a surprise encounter between myself and the chimpanzees at close quarters. But nothing of that sort happened. The two large chimps simply continued to gaze at me $[\ldots]$ and after a few more moments, the two calmly began to groom one another. Without any doubt whatsoever, this was the proudest moment I had known. I had been accepted by the two magnificent creatures grooming each other in front of me. $(S M, 2)$

Die Toleranz der Beobachterin ,,at close quarters“, der anhaltende Blick auf sie und die Ruhe der Schimpansen sind für Goodall Anzeichen eines Erfolgs, den sie sich hart erarbeitet hat. Daher ist die in einer schimpansischen Geste zur Schau

\footnotetext{
${ }^{45}$ Trotz des zweieinhalb Jahrzehnte spannenden Veröffentlichungszeitraum der Texte sind sie alle einer wissenschaftshistorischen Phase zuzuordnen: Die drei Autorinnen begannen ihre Langzeit-Feldforschungsprojekte 1960 (Goodall), 1966 (Fossey) und 1971 (Galdikas). Bekannt wurden die drei Forscherinnen zunächst durch die - mit der Vergabe von Fördermitteln verbundene - Berichterstattung im National Geographic-Magazin, die das öffentliche Bild von den drei Primatologinnen deutlich beeinflusst hat. Vgl. Goodall: My Life Among Wild Chimpanzees; van Lawick-Goodall, Jane: New Discoveries Among Africa's Chimpanzees. In: National Geographic 127 (1965), S. 802-831; Fossey, Dian: Making Friends With Mountain Gorillas. In: National Geographic 137 (1970), S. 48-67; Fossey, Dian: The Imperiled Mountain Gorilla. A Grim Struggle For Survival. In: National Geographic 159 (1981), S. 501-523; Galdikas-Brindamour, Biruté: Orangutans, Indonesia's ,People of the Forest‘. In: National Geographic 148/4 (1975), S. 444-473; Galdikas, Biruté M. F.: Living With the Great Orange Apes. In: National Geographic 157/6 (1980), S. 830-853. Die Monographien berichten von der relevanten Zeit im Feld, erscheinen jedoch teilweise Jahrzehnte nach den darin berichteten Ereignissen und bieten damit einen größeren Reflexionsrahmen an.

${ }^{46}$ In In the Shadow of Man wird dieses Schimpansenmännchen als ,David Graybeard', in späteren Publikationen jedoch als ,David Greybeard' geführt. Ich verwende hier die jeweils durch den jeweiligen Text vorgegebene Schreibweise.
} 
gestellte Akzeptanz, „the proudest moment I had known“. David Graybeard traut sich zudem als erster Schimpanse in die Nähe ihres Camps und nimmt ebenfalls als Erster eine Banane aus Goodalls Hand entgegen (66 f.). All diese Momente sind für Goodall von besonderer Bedeutung und werden hinsichtlich ihres emotionalen Erlebens und der affektiven Reaktionen beschrieben. Bereits in ihren ersten Forschungsmemoiren, In the Shadow of Man, hebt sie eine solche Passage besonders hervor, indem sie den Text mit ihr enden lässt:

One day, as I sat near him at the bank [...] I saw a ripe and red palm nut lying on the ground. I picked it up and held it out to him on my open palm. He turned his head away. When I moved my hand closer he looked at it, and then at me, and then he took the fruit, and at the same time held my hand firmly and gently in his own. As I sat motionless he released my hand, looked down at the nut, and dropped it on the ground.

At that moment there was no need of any scientific knowledge to understand his communication of reassurance. The soft pressure of his fingers spoke to me not through my intellect but through a more primitive emotional channel: the barrier of untold centuries which has grown up during the separate evolution of man and chimpanzee was, for those few seconds, broken down.

I was rewarded far beyond my greatest hopes. (268)

Vorab bereits gab es in Goodalls Forschungsmemoiren etliche Passagen, die Fortschritte im Etablieren einer Forschungsbeziehung oder andere Vertrauensbeweise seitens der Affen wiedergaben. Auch ist dies nicht die erste Stelle, an der eine Berührung durch einen Affen auftaucht; Berührungen wurden vorab sogar als problematisch diskutiert (138 f.). Doch Goodalls In the Shadow of Man läuft auf genau diese Passage hinaus; diese Szene bildet den Abschluss ihres Berichtes und wird in ihrem spirituell inspirierten Alterswerk Reason for Hope (1999) wieder aufgenommen und empathisch weiter ausgedeutet. ${ }^{47}$ An dieser prominent gesetzten Stelle am Schluss von In the Shadow of Man gewinnt die Berührung einen spezifischen Wert. Goodall sieht hier in der taktilen Geste eine Kommunikation sich vollziehen, die sie jenseits der intellektuellen Fakultäten ansetzt: ein Fingerdruck als soziale Bestätigung (,reassurance“, $S M$, 168), nicht vermittelt „through my intellect“ sondern ,through a more primitive channel“" (SM, 268), später noch deutlicher ,a language far more ancient than words, a language that we shared with our prehistoric ancestors" $(R H, 81)$. Der affizierende soziale Kontakt wird von Goodall verstanden als Überwindung einer speziesdifferenzierenden Grenze (,the barrier [...] which has grown up during the separate evolution of man and chimpanzee“, $S M, 268$ ), als eine Überbrückung der offensichtlichen Unterschiede (,a language bridging our two worlds“, $R H, 81$ ). Und diese Erfahrung eines Erfühlens der Verbundenheit mit dem schimpansischen Anderen ist ihr wie zuvor der erste Blickkontant wieder Belohnung und Verdienst (,,reward“, SM, 268) für ihre Anstrengungen. Später, in Reason for Hope, beschreibt sie das Erlebnis als

${ }^{47}$ Goodall, Jane/Berman, Phillip L.: Reason for Hope: A Spiritual Journey. New York 1999, S. 81. Im Folgenden, wenn zur Unterscheidung nötig, als $R H$ geführt; Seitenangaben im Text. 
affektiv tief berührend („And I was deeply moved“, 81) und greift im Dienste der Eindrücklichkeit auf die Herz-Metaphorik zurück: „I [...] stayed there quietly by the murmuring stream, holding on to the experience so that I could know it in my heart forever" (81).

Auch bei Dian Fossey steht die Überwindung einer Barriere bei der Erforschung des anderen Wesens bzw. der anderen Spezies im Mittelpunkt. Diese Barriere wird überkommen durch ein zweiteiliges Erlebnis, welches ebenfalls einer Dramaturgie von Blick zu Berührung, von Akzeptanz zu Vertrauen folgt, wie sie auch Goodalls Erlebnisse mit David Graybeard strukturiert. Hier ist es das junge Gorillamännchen Peanuts, welches Fossey mit der „most rewarding experience“ (GM, 141) überrascht und den Beginn eines neuen Abschnitts in ihrem Forschungsaufenthalt markiert. Dies vollzieht sich in Gorillas in the Mist in zwei kurzen Szenen des Kontakts:

\begin{abstract}
The first occasion when I felt I might have crossed an intangible barrier between human and ape occurred about ten months after beginning the research at Karisoke. Peanuts, Group 8's youngest male, was feeding about fifteen feet away when he suddenly stopped and turned to stare directly at me. The expression in his eyes was unfathomable. Spellbound, I returned his gaze - a gaze that seemed to combine elements of inquiry and acceptance. Peanuts ended this unforgettable moment by sighing deeply, and slowly resumed feeding. Jubilant, I returned to camp and cabled Dr. Leakey I'VE FINALLY BEEN ACCEPTED BY A GORILLA.

Two years after our exchange of glances, Peanuts became the first gorilla ever to touch me. I felt unusually compelled to make this particular day outstanding because the following morning I had to leave for England for a seven-month period to work on my doctorate. [...] Peanuts' bright eyes peered at me through the latticework of vegetation as he began his strutting, swaggering approach. Suddenly he was by my side and sat down to watch my ,feeding 'techniques as if it were my turn to entertain him. When Peanuts seemed bored with the ,feeding' routine, I scratched my head, and almost immediately, he began scratching his own. Since he appeared totally relaxed, I lay back in the foliage, slowly extended my hand, palm upward, then rested it on the leaves. After looking intently at my hand, Peanuts stood up and extended his hand to touch his fingers against my own for a brief instant. Thrilled at his own daring, he gave went to his excitement by a quick chestbeat before going off to rejoin his group. Since that day, the spot has been called Fasi Ya Mkoni, , the Place of Hands'. The contact was among the most memorable of my life among the gorillas. (141 f., Hervorheb. im Original)
\end{abstract}

Auch hier ist es wieder eine (zeitlich weit gestreckte, im Text aber kondensierte) dramatische Steigerung vom Blickkontakt als Zeichen der Akzeptanz, wie Fossey enthusiastisch und legendär ${ }^{48}$ an Leakey schreibt, hin zur Berührung als Symptom einer veränderten Beziehung zwischen Forscherin und Beforschtem, in der die Beobachterin dem eigenen Erkenntnisgegenstand gegenüber als Unterhaltungsmöglichkeit auftritt. Auch diese Passage ist dramaturgisch angeordnet, sowohl als Szene des taktilen Erstkontakts als auch hinsichtlich ihrer Stellung im Text: Ungefähr in der Mitte von Fosseys Bericht in einem Kapitel über Fusionen und

\footnotetext{
${ }^{48} \mathrm{Vgl}$. beispielhaft für die Rezeption dieser Passage RE, S. 389 sowie Abschn. 2.5.2.
} 
Fissionen der beobachteten Gorillagruppen unter der Ägide hervortretender männlicher Protagonisten platziert, ${ }^{49}$ beleuchtet sie innhalb der Erzählung des Kapitels einen dieser Protagonisten näher und stellt die beschriebene „Habituation“ (142) als Grundlage für die Möglichkeit der primatologischen Feld-Beobachtungen heraus (vgl. auch Abschn. 2.3 dieser Arbeit).

Biruté Galdikas' Erfahrungen unterscheiden sich insofern von denen der anderen beiden ,Trimates ' als die Orang-Utans, mit denen sie es primär zu tun hat, auszuwildernde Waisen sind. Als solche suchen sie Kontakt und einen warmen Körper als Ersatzmutter. Dennoch stellt auch Galdikas an den Beginn ihrer Forschungsmemoiren Reflections of Eden die unerwartete Berührung:

\begin{abstract}
Akmad and I were alone by the edge of the great forest of Borneo [...]. Akmad had just given birth, and her gentle, elongated orangutan face with its delicately etched features looked tired. The light of the late afternoon sun shone eerily through Akmad's long auburn hair, silhouetting her form in an incandescent halo.

I wanted to photograph the tiny, wrinkled, nude face of Akmad's newborn infant. [...] Moving my hand gently, I shifted the little infant. [...] Despite my touch, the infant rested quietly in her mother's arms. Akmad's liquid brown eyes remained expressionless. She seemed unaware of my hand on her newborn. Her arm brushed carelessly across my leg as she reached for a pineapple, almost as if I didn't exist. [...] Emboldened, I carefully moved the infant into a better position for a photograph in the fading light. As I gently tugged her, the infant squealed. Akmad's opaque, inner-directed gaze did not change. I glanced at Mr. Achyar [the caretaker] who was watching me intently. The wonder on his face was palpable. I, too, was amazed. Up to that moment I had never imagined the degree to which Akmad accepted me. (3 ff.)
\end{abstract}

Die herausgestellte familiäre Nähe zum Orang-Utan-Weibchen Akmad weist über das einfache Konstatieren eines Grades an Vertrautheit hinaus. Der Moment der von der Äffin geduldeten Berührung ist hier Anlass für eine entscheidende, epistemologische Annahmen revidierende Erkenntnis, die Galdikas mit sich selbst auszumachen hat:

In a moment of absolute clarity I realized the intensity of the bond that I had forged with Akmad. She had singled me out for this unique historic honor: I was a female of another species, but as her bonded mother I had been granted the privilege of sharing her newborn infant. I was probably the first human being in history who was truly an orangutan infant's grandmother. [...] It had taken me more than a decade of living with orangutans [...] to understand finally that orangutans are not just simpler versions of ourselves. [...] What I had taken as indifference and rejection was the orangutan expression of acceptance. I had measured orangutans by human standards of sociability, and had misunderstood. In that moment everything I had been through - the heat, the mud, the humidity, the torrential rains, the fire ants, the leeches, the cobras, pythons, and pit vipers, the fevers, the deaths, the frustrations - became insignificant. I knew that my journey, my personal odyssey into the uncharted depths of the rain forest and the orangutan mind, started earlier, had truly begun. (16)

\footnotetext{
${ }^{49}$ Vgl. „The Natural Demise of Two Gorilla Families: Groups 8 and 9“ (GM, S. 138-151).
} 
Die Reaktionen des Orang-Utan-Weibchens geben keinen Hinweis auf eine emotionale Bindung, sie sind ,tired“, „,expressionless“, „,unaware“, „,careless[]“, usw. Anders als bei den anderen beiden Primatologinnen handelt es sich hier nicht um eine aktive taktile Geste des Affens, die der Forscherin Aufschluss über die Beziehung zum tierlichen Anderen gibt. Hier ist es, scheinbar dem Verhalten der Spezies entsprechend, ein Gewährenlassen, das nah am Ignorieren liegt, das der Forscherin aber zeigt, zu welchem Grad sie akzeptiert wird.

Im ,Drama of Touch', das alle drei Forscherinnen in den zitierten Passagen darbieten, sieht Donna Haraway in ihrer Studie zur Primatologie eine Codierung der für ein westliches Natur-Kultur-Verständnis wesentlichen, geschlechtlich konnotierten Vorstellung von einem privilegierten weiblichen Zugang zur Natur. Für Haraway ist es kein Zufall, dass es drei weiße, junge Frauen sind, die in den 1960er und 1970er Jahren den Kontakt zu Menschenaffen durch eine Berührung herstellen: ,[N]ature approaches man through white woman. Man is placed in nature through his emissary, woman, just as the apes were placed in culture through a human female emissary“. ${ }^{50}$ Die Geste zwischen Tier und Frau ist für Haraway ebenfalls ein Modus der Kommunikation ,,about boundary crossing, about the drama of touch across difference". ${ }^{51}$ Sie kreiert aber ihrer Ansicht nach einen zeitlosen Raum, in dem die lange Tradition einer Vergeschlechtlichung von Natur und Kultur vergessen gemacht wird, gerade indem deren Charakteristika hier aufs Deutlichste durchgespielt werden. Die weiblichen Forschenden erfüllen darin ihre Rolle durch den dreifachen Code von ,Gender, Science, Race", wie Haraway anhand der National Geographic-Narrative beschreibt: In diesen Magazinartikeln und Filmen werden die drei Frauen qua Geschlecht als naturnah und qua Beruf(ung) Wissenschaftlerin als Vermittlerinnen präsentiert, die Insignien westlicher Kultur (Khaki-Shorts, Jeans, Zigaretten) in die Wildnis tragen und durch ihre Nähe zum Tier eine Brücke schlagen. Die Affen werden in diesem Verfahren zugleich in dem Maße aus der Natur geholt und zu Kulturerzeugnissen gemacht, wie menschliches Verhalten und menschliche Biologie durch ,den Affen ' anthropologisch in der Natur verortet werden. Der Fokus auf die drei weißen Frauen der angelsächsischen Sphäre dient der sichtbaren Repräsentation westlicher Kultur: „Woman was closer to nature than man, and that was required in the story, but woman has to be white, or the closeness would have underlined a different and disturbing construction of nature", 52 wie Haraway es formuliert. Das Bewusstsein für eine rassistische Konnotation von Hautfarbe und Animalität ist zeithistorisch gegeben, jenes für die (De-)Konstruktion weiblicher Naturnähe noch nicht. Im Vordergrund des Interesses und der Unterstützung, die die National Geographic Society für die Pionierinnen der primatologischen Feldforschung aufbringt, steht etwas anderes: Diese Narrative sind für Haraway im Wesentlichen

\footnotetext{
${ }^{50}$ Haraway: Primate Visions, S. 149.

${ }^{51}$ Haraway: Primate Visions, S. 149.

${ }^{52}$ Haraway: Primate Visions, S. 154.
} 
Heilsgeschichten, in denen die Menschheit durch weibliche, weiße Botschafterinnen eine Rückkehr ins verlorene Paradies eines Naturzustandes, zumindest aber eine Form der ,survival on earth" 53 sucht: ,,[] rrespective of their personae in other publications or their own agenda for the NGS media" 54 spielen Goodall, Fossey und Galdikas in den Magazin-Artikeln in Text und Bild ,,a gender-coded rhetorical role “55 in einem Propaganda-Stück über Natur als Heilsort für die drängenden sozialen, wirtschaftlichen und ökologischen Probleme des 20. Jahrhunderts. Julika Griem sieht denn auch die wissenschaftlich gestützte und publizistisch inszenierte Verringerung der ontologischen Distanz zwischen Affe und Mensch fundiert durch ein solcherart politisches Projekt. Das ,Drama of Touch' ist in Griems Lesart häufig eine Idealisierung des Affen, die die einstmals skandalöse „Berührung von Affenpranke und weißem Frauenkörper ${ }^{\text {"56 }}$ in King Kong ${ }^{57}$ umwertet in eine Variation von Michelangelos Motiv „zweier zur Berührung ausgestreckter Finger“. 58 Die Geste wird zur „Pathosformel einer in mehrerer Hinsicht ,berührenden“ Begegnung von Affen- und Menschenhand" ${ }^{59}$ Griems Hinweis auf den Begriff der ,Pathosformel' ist dabei von Interesse: Nach Ulrich Port geht es bei der Pathosformel zwar ,immer um einen (potentiell) theatralen Ausdruck starker affektiver Erregung, um Körperbilder und mit ihnen korrespondierende Sprachgebärden“, ${ }^{60}$ wie sie Griems Lesart entgegenkommt. Im weiteren Sinn handele es sich bei der Pathosformel jedoch um den „Problemtitel für alle affekt- und passionsbezogene Motive, Topoi und Codes, die ein bestimmtes Niveau der Formalisierung erreicht haben und in der Lage sind, künstlerische Darstellungen wie theoretische Argumentationen zu strukturieren ". ${ }^{61}$ In diesem Verständnis lassen sich die Dramen der Berührung, die die Primatologinnen inszenieren, weniger in Griems Sinn als Indikatoren einer romantisierenden Beziehung zum Affen denn als formelhafte Instrumentarien einer Argumentationsstruktur im Medium des Forschungsberichts lesen. ${ }^{62}$

\footnotetext{
${ }^{53}$ Haraway: Primate Visions, S. 150.

${ }^{54}$ Haraway: Primate Visions, S. 157.

${ }^{55}$ Haraway: Primate Visions, S. 157.

${ }^{56}$ Griem: Monkey Business, S. 228.

${ }^{57}$ Cooper, Merian C./Schoedsack, Ernest B.: King Kong. Radio Pictures 1933.

${ }^{58}$ Griem: Monkey Business, S. 228.

${ }^{59}$ Griem: Monkey Business, S. 228.

${ }^{60}$ Port, Ulrich: Pathosformeln. Die Tragödie und die Geschichte exaltierter Affekte (1755-1888). München 2005, S. 27.

${ }^{61}$ Port: Pathosformeln, S. 27.

${ }^{62} \mathrm{Im}$ Blick hat Griem bezeichnenderweise vor allem die bildliche Gestaltung einer solchen Begegnung auf dem Umschlag von Peter Høegs Die Frau und der Affe und in Michael Apteds Verfilmung von Gorillas in the Mist und nicht die Schilderungen der Forschungsmemoiren. Vgl. Griem: Monkey Business, S. 228.
} 


\subsubsection{Das ,Drama of Touch' als Medium fühlender Erkenntnis}

Die drei Monographien von Goodall, Fossey und Galdikas bieten denn auch einen anderen Rahmen für diese Geste der Berührung als die Popularisierung der Primatologie durch die National Geographic Society und ihr Magazin oder die populärkulturelle Appropriation der Primatologie in Bild und Film. In In the Shadow of Man, Gorillas in the Mist und Reflections of Eden, so noch einmal die These, werden diese Dramen der Berührung durch die Dramaturgie der Forschungsberichte in zweifacher Hinsicht als Medium der Erkenntnis im Forschungsprozess instrumentalisiert: Sie dienen in ihrer außergewöhnlichen Konstellation des taktilen Kontaktes zwischen Mensch und Tier sowohl als Nährmedium für die Entstehung affektiver Erkenntnis, als auch als Kommunikationsmittel, das einerseits zwischen Mensch und Tier, andererseits aber auch zwischen dem wissenschaftlichen Subjekt und seinen affektiven Erkenntnismöglichkeiten vermittelt.

Jane Goodall setzt mit der Positionierung der Berührungs-Szene nicht nur einen Schlussakzent, sie nimmt auch eine oppositionelle Anordnung zwischen der wissenschaftlichen Wissensaneignung und der emotionalen Vermittlung vor. „Scientific knowledge“ (SM, 268), das Sammeln von Daten und Beobachtungen ist Thema des Forschungsberichts und Verdienst von Goodalls Langzeitforschung. Wie sie hier jedoch deutlich macht, ist die Basis dafür eine Verständigung zwischen Forscherin und Beforschtem, die sich nicht durch reziproke Wissensanhäufung herstellt, sondern nur durch die emotionale Vermittlung einer Übereinkunft („Du darfst mich beobachten, wenn Du mich in Ruhe lässt.“ - „Ich will Dich nur beobachten, um mehr über Dich zu wissen. Von mir geht keine Gefahr aus.") über die speziesdifferenzierende Grenze hinweg, die Mensch und Schimpanse trennt (siehe auch Abschn. 2.4). Mit dieser Einsicht verabschiedet Goodall ihre Leserschaft aus ihrem Forschungsbericht.

Fosseys Wortwahl und Fokus auf die Individualität der beobachteten Tiere und ihre Belange lässt in der Passage aus Gorillas in the Mist zugleich einen Subtext entstehen, der das Sinnliche auf eine andere Ebene rückt, und die Begegnung auch als erotische aufscheinen lässt, wie dies in der populären Fossey-Rezeption gern gelesen wird. Der Blick des jungen Männchens, „unfathomable“ (141), hält Fossey gebannt (,Spellbound“,141), bis sie seinen Blick erwidert, und es zu einem flirtiven „exchange of glances“ (141) kommt. Dies wird für Fossey zu einem „,unforgettable moment“(141). Sie ist ,jubilant“, und später wird dieser Gorilla in einer eher ungeschickten Formulierung ,the first gorilla ever to touch me“ (141), just bevor sie zu einer längeren Abwesenheit aufbricht. Nach der Berührung bedient sich der Gorilla der mittlerweile vom Affen entlehnten Geste potenzierter Männlichkeit und schlägt sich auf die Brust. Sicherlich lässt sich dies als Flirt mit dem Tier lesen, der der spröden Forscherpersona, die Fosseys Gorillas in the Mist anbietet, eine schillernde erotische Komponente verleiht. Diese mittlerweile bereits traditionell gewordene Lesart möchte ich an dieser Stelle jedoch als unproduktiv zunächst beiseite legen (siehe aber Abschn. 2.5). 
Die Abgrenzung, welche Fossey zwischen Mensch und Gorilla sieht, wird von ihr bezeichnenderweise zunächst als ,intangible barrier“ (141), also als unangreifbar, unantastbar wahrgenommen. Durch den Zweischritt von Blickkontakt, d. h. einem Austausch der Blicke (eben der ,exchange of glances“), und tastender Berührung wird diese Grenze auch nicht aufgehoben, sondern Fossey schreibt konjunktivisch „I might have crossed an intangible barrier“ (141, Hervorheb. MS). Die Grenze bleibt Fosseys Passage zufolge bestehen, sie ließ sich nur vielleicht - „for an instant“ überschreiten. Sie deutet Peanuts' Blickals über die Neugier (,,inquiry“) eines jungen Männchens hinausgehend: Sie liest sie als überindividuelle Geste der Akzeptanz, wie der Telegramm-Text an Louis Leakey zeigt. Doch darf ihr enger Fokus auf das Verhalten und Empfinden der Gorillas, denen sie an dieser Stelle grammatikalisch wie auch programmatisch Priorität einräumt, nicht darüber hinwegtäuschen, welche immense Bedeutung die temporäre Überwindung jener vermeintlich unangreifbaren Barriere durch eine simple Berührung gewinnt. Fosseys ausgestreckte Hand ist ein Angebot, welches Peanuts willig annimmt, und sei es auch nur kurz. Diese Geste ist von so immenser Bedeutung für Fossey, dass sie den Ort, an dem sie vollzogen wurde, ,the Place of Hands“ (142) nennt und somit den taktilen Spezieskontakt toponym werden lässt. Mit dieser Markierung verleiht sie der Szene der Berührung historische Bedeutung.

Für Biruté Galdikas ist das Berührenlassen als Zeichen der Einordnung in die spezifische, matrilineare Familienstruktur der Orang-Utans - hier auf die Spitze getrieben durch die deutliche Stilisierung zur Marienszene (,,an incandescent halo“, $R E, 3)$ - der höchste Verdienst, die Wiedergutmachung für die ausführlich aufgelisteten Schrecken der Wildnis, und zugleich ein Wendepunkt für ihr Verständnis von den Orang-Utans und ihrem Verhältnis zu diesen. Bezeichnenderweise sieht sie dies nicht als Abschluss ihrer Forschung, als finale Einsicht in die Wirkweise eines Orang-Utan-Sozialverhaltens, als publizierbare Erkenntnis einer jahrzehntelang währenden Beobachtungssituation, sondern ganz im Sinne der textuellen Verortung als Auftakt eines Berichts als Anfang einer Forschungsreise jenseits der Preliminarien der Etablierung einer Forschungsbeziehung.

Alle drei Autorinnen institutionalisieren und instrumentalisieren somit die taktile Begegnung als emotionale Vermittlung und als Medium wissenschaftlicher Erkenntnis. Und zugleich gewähren diese Szenen Einblick in die Wirkungsweisen eines emotionalen Registrierens: Die Veränderung in der Beziehung zwischen Beobachterin und Beobachtetem manifestiert sich hier in der Berührung; und diese wird haptisch und zugleich affektiv wahrgenommen, bevor sie erst im Anschluss kognitiv gedeutet und genutzt werden kann. Bruno Gammerls Schichtarbeit am „Gefühl als Getast“63 folgend lässt sich hier eine Strategie erkennen, die Geschlechterproblematik, welche den Ausgangspunkt für die Karriere der drei Autorinnen bildet, in eine eigene Methodik und Rhetorik der Primatologie

${ }^{63}$ Gammerl, Benno: Gefühlte Entfernungen. In: Frevert, Ute u. a. (Hg.): Gefühlswissen. Eine lexikalische Spurensuche in der Moderne. Frankfurt a. M./New York 2011, S. 179-199, hier S. 181, Hervorheb. im Original. 
zu wenden: Alle drei Forscherinnen sind ausdrücklich von Louis Leakey für die Aufgabe der Primaten-Beobachtung aufgrund der Annahme ausgewählt worden, erstens dass Frauen empathiefähiger als Männer seien, und zweitens dass die professionelle Ausbildung durch konventionelle wissenschaftliche Lehre aus den an der Affenforschung beteiligten Disziplinen die notwendige Offenheit dem Untersuchungsgegenstand gegenüber behindere. ${ }^{64}$ Gammerl verweist in seiner Auseinandersetzung mit historisch spezifischen Emotionsbegriffen im Spannungsfeld zwischen Nähe und Distanz auf die Beschreibung von Gefühl als Getast und ,Contactum' im 18. Jahrhundert, die von der physiologischen Dimension der Berührungsempfindung dominiert gewesen sei. In dieser Vorstellung werden Sehen und Fühlen räumlich differenziert: „Während das Sehen eine gewisse Distanz voraussetzte, bedurfte das Fühlen der Nähe“".65 Die durch diese ausschlaggebende Bedeutung der räumlichen Nähe für die Möglichkeit des (Er-)Fühlens gewonnene Fokussierung des Gefühls auf den Tastsinn stellt laut Gammerl spätere, psychologisch informierte Emotionsbegriffe auf den Kopf, indem es nur das Materielle, das Greifbare als fühlbar klassifiziert - und etwa das Immaterielle und Subtile nicht. Dieses Verständnis von den haptischen Qualitäten und Bedingungen des Gefühls als eines Vorgangs, „,innerhalb dessen das Subjekt sich seines eigenen Zustands bewusst werde", ${ }^{66}$ weicht im 19. und 20. Jahrhundert immer stärker einer Enträumlichung des Gefühlsbegriffs und einer Differenzierung von körperlichen und geistigen Phänomenen. Selbst im englischsprachigen Lexikon, das länger noch ,feeling' und ,touching ' zusammendenkt und mit sinnlicher Wahrnehmung verbindet, findet im Laufe des 20. Jahrhunderts eine „Entsomatisierung von feeling im Zuge wissenschaftlicher Entwicklungen“"67 statt, bis ,touch“ nur noch als Kettenglied einer etymologischen Erschließung von ,feeling' bestehen bleibt. Interessanterweise vollzieht sich jedoch im 19. Jahrhundert auch jener Zusammenschluss von Weiblichkeit und Gefühligkeit, der eine Differenzierung von distanzvergessener Sentimentalität und empfindsamer Sensitivität als Pole eines Spektrums von (weiblicher) Emotionalität provoziert. ${ }^{68}$ Obwohl die Emotionsforschung seither den Emotionsbegriff und sein Verständnis mehrfach justiert hat, bleibt dieses Spektrum weiterhin an Konzepten westlicher Weiblichkeit haften.

Goodall, Fossey und Galdikas werden für die Affenforschung ausgesucht, weil ihnen aufgrund ihres Geschlechts eine dem Forschungsprojekt förderliche

\footnotetext{
${ }^{64} \mathrm{Vgl}$. Morell: Called ,Trimates', S. 420 f.; Galdikas: Reflections of Eden, S. 49. Beim Zoologen George Schaller findet sich der Hinweis darauf, dass Louis Leakey bereits in den 1950er Jahren zwei kurze Studien von weiblichen Laienforscherinnen, der Sekretärin Rosalie Osborn und der Journalistin Jill Donisthorpe, durchführen ließ, vgl. Schaller: The Year of the Gorilla, S. $18 \mathrm{f}$.

${ }^{65}$ Gammerl, Benno: Gefühlte Entfernungen, S. 181.

${ }^{66}$ Gammerl, Benno: Gefühlte Entfernungen, S. 186.

${ }^{67}$ Gammerl, Benno: Gefühlte Entfernungen, S. 183, Hervorheb. im Original.

${ }^{68}$ Vgl. Gammerl, Benno: Gefühlte Entfernungen, S. 187; zu den Anfängen dieser Entwicklung im 18. Jahrhundert siehe auch Honegger: Die Ordnung der Geschlechter, S. 165 ff.
} 
empfindsame Sensitivität angerechnet wird. Wie Haraway schreibt, ist es ihre Aufgabe als Frau, ,to create reciptivity, to produce the conditions in which the animal can approach. "69 In Aneignung dieser Rolle entwickeln sie ein emotionales Sensorium und eine Methodik empathischer Verhaltensbeobachtung, um dieser Erwartung gerecht zu werden - und werden schließlich oft unter den Verdacht der distanzvergessenen Sentimentalität gestellt. Anlass hierfür bieten gerade die Einzelszenen der Berührung mit einem Tier, deren Modus das anekdotische Erzählen ist. Diesem hatte der britische Zoologe Solly Zuckerman bereits 1932 einen giftigen Nachruf geschrieben, der sich hauptsächlich an Passagen aus Reiseberichten wie jenen Du Chaillus oder Wallace' richtete:

The anecdotal method in animal psychlogy came to an end because it had brought ridicule upon itself by its endless flights into the realms of imagination, and because the experimental method promised results [...] since the conditions in which experiments are made can be controlled and adequately described. ${ }^{70}$

Zuckerman studierte im Labor die Folgen von Kopfverletzungen an Affen im Dienste der Rüstungsforschung, nachdem er das später radikal eingeschränkte Haremsparadigma für Paviane von gefangenen Affen im Londoner Zoo abgeleitet und universalisiert und in Südafrika Paviane geschossen hatte, um aus deren Anatomie ihr Verhalten abzuleiten. Seine Forderung richtet sich dezidiert gegen „sociologists“ (13) und nicht-naturwissenschaftliche Methoden, und plädiert für Berichte „free from emotional bias“ (173). Diese Auffassung herrscht auch noch in den 1960er und 1970er Jahren vor, als sich Goodall, Galdikas, und in etwas beschränkterem Maße auch Fossey einer emotionalen Rhetorik zuwenden, die ihrer Forschungsweise und der Bedeutung ihrer Erkenntnisse für die Wissenschaft entspricht, die jedoch an den Codes eines Wissenschaftsbetriebes zerschellt, der eine hierarchische Differenzierung von ,Emotion“ und ,Ratio ' bis in den Sprachgebrauch hinein durchsetzt. ${ }^{71}$

\subsubsection{Das ,Drama of Touch`als Anlass für Selbstreflexion}

Das ,Drama of Touch' ist damit in den hier untersuchten Forschungsmemoiren textueller Beleg einer methodischen Arbeit an der Nähe zum Tier und der Früchte,

\footnotetext{
${ }^{69}$ Haraway: Primate Visions, S. 149, Hervorheb. im Original.

${ }^{70}$ Zuckerman, Solly: The Social Life of Monkeys and Apes. London 1932, S. 11. Im Folgenden, wenn zur Unterscheidung nötig, als $S L$ geführt; Seitenangaben im Text. Siehe zu Zuckerman und einer weiteren Ausdeutung dieses Zitats auch Abschn. 4.1.1.

${ }^{71}$ Siehe auch Ziswiler, Vincent: Erzählen in den biologischen Wissenschaften einst und jetzt. In: Balz Engler (Hg.): Erzählen in den Wissenschaften. Positionen, Probleme, Perspektiven. Fribourg 2010, S. 66-81.
} 
die diese trägt. Es ist repräsentativ geworden für die Art von primatologischer Feldforschung, die die drei weiblichen Gründungsfiguren angestoßen haben. Dies ist durchaus problematisierbar. Shirley C. Strum, als Washburn-Schülerin ebenfalls in einer Art Gründerinnenposition in der Feldforschung mit Pavianen, vollzieht durch ihr eigenes, Drama of Touch“ in den Forschungsmemoiren Almost Human eine ambivalente Selbstreflexion. ${ }^{72}$ Keinesfalls will sie ,the stereotype the media loved to portray“, „Miss Whosits among the Wild Whatsits [...] in love with Nature“ (61) sein. Doch sie macht ihre Rechnung ohne die Paviane, die sie beobachtet:

Baboons have an elaborate system of communication: their gestures, postures, facial expressions and sounds convey a wide range of emotions. By standing in a certain place and not doing anything, I, too, was communicating. Nonetheless, I was drawn further into the social network of the baboons than I had ever intended to go. I realized this one day when I was once again following the drama of the Naomi-Ray friendship. I sat down at baboon level to watch them as they became engrossed in grooming. [...] Suddenly I felt a gentle touch on my back, too light to be the rough grass rubbing against my thin cotton T-shirt, and too strong to be an insect crawling inside it. I turned around quickly - and startled little Robin, Naomi's juvenile daughter, who was a few inches behind me. She retreated quickly, and I, just as quickly, figured out what had happened. Robin was so relaxed with me because I always seemed to be around her mother, and she had approached me quietly and tentatively begun to groom the lower part of my back. It was a gesture so intimate that it touched and thrilled me. But I was also upset that I had let it happen, worried that it might permanently change my relation to the troop and ruin my stand against interaction. At the same time it seemed to me incredible that a baboon had trusted me enough to cross the many barriers between us. (57 f., Hervorheb. im Original)

Die Berührung ist hier unerwünschtes Nebenprodukt einer Nähe, die ausdrücklich den Körperkontakt ausspart. Auch Shirley Strum nutzt die Zuspitzung des Erlebnisses dramaturgisch, um etwas in ihrem autobiografischen Forschungsbericht zu verdeutlichen: Die Szene ist Teil eines mit „Changes“ betitelten frühen Kapitels, in dem sie festhält, wie sie selbst und ihre Forschungshaltung sich durch die Feldarbeit - und durch die beobachteten Paviane - verändert haben. Strum bezieht in Abgrenzung zu Goodall, Fossey und Galdikas Position gegen die Interaktion mit den beobachteten Tieren, weil sie ihren Untersuchungsgegenstand so wenig wie möglich beeinflussen und zugleich sich selbst nicht den sozialen Regeln der Paviangruppe aussetzen will. Gleich zu Beginn des Kapitels hält sie fest, es sei zwar „extremely tempting to reach out across the species chasm to touch and communicate with the baboons“ (54), aber: „I had come to Africa to do my research project; I had no intention of getting involved with the animals. Both my philosophy and my behavior emphasized my distance“ (54). Doch der erste Satz dieses Kapitels lautet bereits proleptisch: „I was changing“ (54). Denn ebenso wie die anderen drei Forscherinnen ist Strum darauf angewiesen, zumindest räumliche Nähe zu

\footnotetext{
${ }^{72}$ Strum, Shirley C.: Almost Human. A Journey into the World of Baboons. New York 1987. Im Folgenden, wenn zur Unterscheidung nötig, als $A H$ geführt; Seitenangaben im Text.
} 
den Tieren zu schaffen, um diese ungehindert und über längere Zeit beobachten zu können. Anders als Zuckerman weiß Strum, dass sich aus den ,hard facts ‘ der Anatomie nur begrenzt Aufschluss über das Sozialleben und Verhalten der Affen verschaffen lässt. ${ }^{73}$ Dazu benötigt es lebende Tiere in ihrem natürlichen Umfeld. Doch damit findet auch sie sich den sozialen Bezügen innerhalb der Gruppe ausgesetzt. Die räumliche Konstellation und die zeitliche Präsenz provoziert hier eine Berührung, die Strum in ihren taktilen Qualitäten beschreibt (,,a gentle touch on my back, too light to be the rough grass rubbing against my thin cotton T-shirt, too strong to be an insect", 57), um Realitätstreue zu schaffen. Sie liest diese Berührung zwar als Vertrauensbeweis (,intimate“, 57; ,incredible that a baboon had trusted me enough“, 58) und registriert sie affektiv. Strum scheidet jedoch zwei emotional-kognitive Reaktionen voneinander: Ist sie auf der einen Ebene „touched and thrilled“, ist sie auf der anderen „upset“ und „worried“ (57). In diesem Drama versucht sie erst analytisch zu verstehen, wie es zu dieser Szene kommen konnte, um dann zu überschlagen, welche Folgen der unbeabsichtigte Verstoß gegen ihr eigenes wissenschaftsethisches Reglement nach sich ziehen könnte, und welche Art von Verhalten als Reaktion angemessen ist. Auch in diesem ,Drama of Touch“ offenbart sich eine Methodik und ein emotionales Registrieren, das sich hier stärker noch als bei den anderen drei Forscherinnen selbstreflexiv auswirkt. Strums Erkenntnisgewinn betrifft die eigene Situation und Positionierung; der Moment der körperlichen Nähe ist für sie ,persönlich ' berührend, ,professionell jedoch bedrohlich: Die Grenze zwischen den Spezies und zwischen Beobachterin und Beobachteten ist hier nicht nur eine Barriere wie bei Goodall und Fossey, sie ist ein Abgrund (,chasm“, 54). Dieser Abgrund, das stellt Strum durch ihre Ausführungen heraus, ist in der Unkommunizierbarkeit des Sozialen, die er zunächst zu bergen scheint, auch ein schützender Festungsgraben: „Should they view me as another baboon, one with whom they could exchange touches and greetings, they would also see me as being able to accept their aggression, which I could not do" (54).

In der Folge, so berichtet Strum, wird sie ,much more alert to possible overtures from the animals" (58) und ist bemüht, den zunehmenden Avancen der Paviane auszuweichen, während sie doch feststellen muss, dass selbst ,,[n]ot interacting“ (59) und ,just being with them“ (54) zu ,,[a]ttachment, although troublesome“ (59) und einem „strong emotional bond“ (54) führen. Strum will sich dem Stereotyp emotional sensibler, naturnaher Weiblichkeit entziehen („I wanted none of it.“, 61), welches in der medialen Inszenierung von Goodall, Fossey und Galdikas zeitgenössisch bereits etabliert ist. Zugleich jedoch entwickelt auch sie eine ähnliche, auf emotionaler und räumlicher Nähe zum Tier basierende Arbeitsweise. In dieser spielen Affekte eine Rolle als epistemische Instrumente nicht nur des Verständnisses der

\footnotetext{
${ }^{73}$ Wie noch auszuführen sein wird (Abschn. 4.1) ist auch Zuckerman sich dieses Problems eigentlich bewusst und ergänzt seine anatomischen Untersuchungen um ethologische Beobachtungen einer Zoo-Population in London.
} 
sozialen Prozesse innerhalb der Paviangruppe, denen Strum teilweise im ,Drama of Touch“ selbst ausgesetzt ist, sondern auch der Selbstreflexion. Das „life as a field observer“ (59) beschert ihr nicht allein ,attachment“ als ,unexpected reward[]“ (59) sondern auch ,integration“ (59). Damit meint sie hier anders als Galdikas vor allem eine Selbstkonstitution:

Up until this point, my life had always been fragmented. [...] But now there was only one thing I needed to contentrate on: watching baboons, thinking about baboons, writing about baboons, organizing my data on baboons, reading about baboons and of course, eventually dreaming about baboons. (59)

Die Feldbeobachtung biete ,intellectual and physical challenge“ (59): „[E]ach evening mentally and physically exhausted, but wonderfully so" (59), habe sie in ihrer Forschungsaufgabe zu sich selbst gefunden. Die Szenen des ,Drama of Touch“ der Primatologie verdeutlichen nicht nur physische und emotionale Nähe zu Schimpansen- und Gorilla-Männchen, Orang-Utan-Weibchen und Pavian-Jungtieren, sondern hier auch eine Selbstbegegnung in der Übereignung an das Forschungsvorhaben als Lebensaufgabe.

\subsection{Das Erhabene im Gorilla: Ästhetische Reflexion und affektive Erkenntnis im Feld}

Die ,Trimates“ und ihre Zeitgenoss/innen schöpfen ihre emotionszugewandte Forschungsmethodik nicht aus dem Leeren. Feldprimatologische Forscher/innen können auf ein Modell epistemologisch nutzbarer Emotionalität zurückgreifen, das bereits eine lange abendländische Tradition besitzt und einen Gewährsrahmen für affektgeleitete Erkenntnis bietet. Ein solches Modell ist der ästhetischen Reflexion zu entnehmen und lässt sich als Beispiel für eine Nutzbarmachung emotionalen Erlebens für die Wahrnehmung und Wissensgenerierung erkunden. Es ist das Gefühl des Erhabenen.

Der Konnex des im Kontext von Theorien des Schönen diskutierten Gefühls des Erhabenen mit der wissenschaftlichen Forschung an Affen, die im okzidentalen Verständnis gemeinhin unter die Kategorie des Hässlichen fallen, mag zunächst überraschend wirken. Fallen doch Tiere, besonders der Affe mit seinem „höhnische[n] Aufenthalt an der Schwelle der aufgerichteten Menschengestalt““ 74 als Instanzen einer Ästhetik des Hässlichen auf:

Bei dem Thier dagegen, es ist nicht zu leugnen, erzeugen sich Formen von ursprünglicher Häßlichkeit, die ihren Gräuelanblick durch keinen komischen Zug aufheitern. [...] Gewisse Quallen, Sepien, Raupen, Spinnen, Rochen, Eidechsen, Frösche, Kröten, Nager,

\footnotetext{
${ }^{74}$ Vischer, Friedrich Theodor: Aesthetik oder Wissenschaft des Schönen. Zum Gebrauche für Vorlesungen. Bd. 2,1: Die Lehre vom Naturschönen. Reutlingen/Leipzig 1847, S. 159 f.
} 
Pachydermaten, Affen, sind positiv häßlich. [...] Wir sehen, daß die häßlichen Formen sich vorzüglich auf den Uebergängen der Thierreiche erzeugen, weil auf ihnen sich ein gewisser Widerspruch, ein Schwanken zwischen verschiedenen Typen auch in der Gestalt kund geben $\operatorname{muß}[.]^{75}$

Und doch drängt sich durch seine Repräsentation in den primatologischen Forschungsmemoiren (und darüber hinaus) gerade der Berggorilla als Tier des Erhabenen auf. Unterschiedliche Affenarten bieten unterschiedliche Forschungssituationen und verschiedene emotionale Szenarien. Schimpansen sind sozial sehr interaktive, laute und durchaus gewalttätige Tiere, die die Aufmerksamkeit der Beobachtenden anders beanspruchen als die eher langsamen, einzelgängerischen und scheuen Orang-Utans in den dichten Wäldern Borneos. Aufgrund ihrer vergleichsweise ruhigen sozialen Interaktion und ihres Lebensraumes an den Hängen der Vulkanberge in Zentral- und Ostafrika dienen Berggorillas jedoch, wie sich zeigen wird, als Paradigma für das Erhabene am Affen. Dieses und andere Formen des Erhabenen, die sich in den Texten der Gorilla-Forschung finden, sollen im Folgenden beispielhaft als Modell emotionaler Wahrnehmung und Erkenntnis in der Primatologie untersucht werden. Dazu ist jedoch zunächst eine Herleitung eines Begriffs vom Gefühl des Erhabenen nötig, wie er in Folge der postmodernen Diskussion des kantischen Erhabenen entsteht, bevor anhand dreier Texte das primatologische Erhabene am Gorilla und sein Wandel in der Postmoderne herausgearbeitet werden können.

Die Intertextualität, mit der die drei Gorilla-Texte arbeiten, zeigt, wie weitreichend und verankert die Erkenntnisgewinnung durch das Gefühl des Erhabenen ist: Dian Fosseys unmittelbarer Vorgänger in der Gorilla-Freilandforschung ist der deutsch-amerikanische Zoologe George Schaller. In seinem 1964 erschienenen The Year of the Gorilla bezieht er sich namentlich auf die großen Afrikareisenden und -forscher (z. B. auf Hanno, 13; von Beringe, 17; von Götzen, 25; und Du Chaillu, 15 u. 210) ebenso wie auf die Teilnehmer eines Diskurses über den Affen (u. a. Buffon, 216; Monboddo, 221; und Thomas H. Huxley, 221) und repliziert teilweise deren Reiserouten, Eindrücke oder Argumente. Dian Fossey weiß um George Schallers Vorleistungen und referiert auf diesen ebenso wie auf Carl Akeley, dessen Grab sie besucht (GM, 3 f.). In Gorillas in the Mist ist das erste Kapitel nach den beiden Vorbildern benannt als „The Meadow of Akeley and Schaller“ (1). Der Neurobiologe Robert Sapolsky schließlich forscht eigentlich in Ostafrika mit Pavianen. Er reist jedoch knapp sechs Monate nach Fosseys Ermordung Mitte der 1980er Jahre in die Virunga-Berge, um auf ihren Spuren zu wandeln. Diese Reise nutzt Sapolsky

\footnotetext{
${ }^{75}$ Rosenkranz, Karl: Aesthetik des Häßlichen. Königsberg 1853, S. 21 f. Bereits Edmund Burkes ,Phänomenologie" des Erhabenen setzt jedoch das Erhabene und das Schöne in Spannung zueinander, sieht Hässlichkeit als Möglichkeit für das Erhabene, wo sie ,terror' auslöse und verweist mehrfach auf Tiere als Instanzen des Erhabenen, bspw. bezüglich der „Cries of Animals“. Burke, Edmund: A Philosophical Enquiry into the Origin of our Ideas of the Sublime and Beautiful. London ${ }^{2} 1759$, S. 155 f. u. 225.
} 
in seinem A Primate's Memoir ${ }^{76}$ als Anlass zur Diskussion von Fosseys Persönlichkeit und Forschungsarbeit ebenso wie zur Reflektion über die eigene Arbeit mit den Pavianen. So wie die Autor/innen von den Berichten und Erfahrungen ihrer Vorgänger/innen profitieren und hier eine wissenschaftliche Notwendigkeit - den Forschungsüberblick - berücksichtigen, nutzen sie diese auch zur Reflexion, zur Erkundung der eigenen Position - und zur Erweiterung der eigenen epistemischen Möglichkeiten. Zentral dabei sind der Gorilla und sein Habitat als Topos einer Selbsterkenntnis, die durch das Gefühl des Erhabenen indiziert wird. Dieser Topos der Selbsterkenntnis, so soll abschließend anhand von Lukas Bärfuss' Roman Hundert Tage ${ }^{77}$ verhandelt werden, lässt sich mit dem Blick der Literatur im 21. Jahrhundert kritisch als postmoderne Egozentrik lesen.

\subsubsection{Das Gefühl des Erhabenen als Modell affektiver Erkenntnis}

Spätestens mit seiner Renaissance als postmodernem philosophischen Gegenstand um 1990 wird das Gefühl des Erhabenen im Rückgriff auf Immanuel Kant in Verbindung gesetzt mit einer Thematisierung der nicht nur empfindungs-, sondern auch affektbasierten Erkenntnis. ${ }^{78}$ Dietmar Till hat diesen Diskurs zwar als mittlerweile versiegtes Modephänomen und postmoderne Verengung der Begriffsgeschichte kritisiert und darauf verwiesen, dass eine Beschäftigung mit dem Erhabenen einer Ergänzung des ,hermeneutische[n] Modell[s] des Gesprächs mit dem Text [...] durch die Einbeziehung der diskursiven Rahmenbedingungen“79 desselben bedarf. Dieser Verweis auf die Funktion, welche dem Erhabenen in den antiken Texten in einem rhetorischen Kontext zukommt, ist ohne Zweifel wichtig. Der Diskurs der 1990er Jahre um das Erhabene scheint jedoch gerade deswegen für die vorliegende Studie anregend, weil er sich auf die aisthetischen und epistemologischen Dimensionen des Erhabenen konzentriert - und somit auch zeigt,

\footnotetext{
${ }^{76}$ Sapolskys A Primate's Memoir erschien 2001 bei Jonathan Cape (London). Im Folgenden wird aus der unveränderten Taschenbuchausgabe von 2002 zitiert: Sapolsky, Robert M.: A Primate's Memoir. New York u. a. 2002. Im Folgenden, wenn zur Unterscheidung nötig, als $P M$ geführt; Seitenangaben im Text.

${ }^{77}$ Bärfuss, Lukas: Hundert Tage [2008]. München 2010. Im Folgenden, wenn zur Unterscheidung nötig, als $H T$ geführt; Seitenangaben im Text.

${ }^{78}$ Eine solche Renaissance des Erhabenen wird zuvorderst von Jean-François Lyotard und seiner Schülerin Christine Pries angeregt und in Zusammenhang mit postmodernem Wissen und den ,neuen Technologien' des ausgehenden 20. Jahrhunderts gesetzt. Vgl. Lyotard, Jean-François: Die Analytik des Erhabenen (Kant-Lektionen, Kritik der Urteilskraft, §\$ 23-29). München 1994 (frz. 1991); Pries, Christine (Hg.): Das Erhabene. Zwischen Grenzerfahrung und Größenwahn. Weinheim 1989.

${ }^{79}$ Till, Dietmar: Das doppelte Erhabene. Eine Argumentationsfigur von der Antike bis zum Beginn des 19. Jahrhunderts. Tübingen 2006, S. 7.
} 
welchen Wandel die Deutung des Erhabenen im Zuge seiner Theoretisierung in der Postmoderne erfährt.

Anders als der von ihm rezipierte und kritisierte Edmund Burke, für den „passions $[\ldots]$ the organs of the mind ${ }^{\prime 80}$ sind, unterhält Kant selbst in der Anthropologie in pragmatischer Hinsicht überwiegend kein gutes Verhältnis zu den Leidenschaften als „durch die Vernunft des Subjektes schwer oder gar nicht bezwingliche Neigung[en]"81 und den Affekten als Gefühlen „einer Lust oder Unlust im gegenwärtigen Zustande, welche[] im Subjekt die Überlegung (die Vernunftvorstellung, ob man sich ihm überlassen oder weigern soll) nicht aufkommen" ${ }^{\text {82 }}$ lassen. Er vergleicht beide mit der Dynamik der Wasserkraft: Der Affekt wirke wie „ein Wasser, was den Damm durchbricht“, die Leidenschaft „wie ein Strom, der sich in seinem Bette immer tiefer eingräbt" ${ }^{83}$ Beiden wird also ein nachhaltiger, verändernder Effekt zugeschrieben. Ihre Wirkung ist krankhaft: Affekte, zu denen Kant die Freude, die Traurigkeit, den Gram, den Schreck, den Zorn und die Scham zählt, ähnelten dem Schlagfluss; ${ }^{84}$ die Leidenschaften, darunter Ehrsucht, Rachsucht, Herrschsucht, aber auch Verliebtsein, ${ }^{85}$ der „Schwindsucht, oder Abzehrung“ ${ }^{86}$ Sie sind gar „Krebsschäden für die reine praktische Vernunft". ${ }^{87}$ Diese emotionstypologische Feindifferenzierung verurteilt Affekte und Leidenschaften somit aufgrund ihres einschränkenden Einflusses auf die Vernunft: ${ }^{88}$ „Affekten und Leidenschaften unterworfen zu sein, ist wohl immer Krankheit des Gemüts; weil beides die Herrschaft der Vernunft ausschließt“" ${ }^{89}$ Folglich spricht sich Kant zugunsten einer „Affektlosigkeit, ohne Verminderung der Stärke der Triebfedern zum Handeln ${ }^{“ 90}$ aus. Es empfiehlt sich also eine depressionslose Apathie als Freiheit und Herrschaft über sich selbst. Weil Kant die Affekte jedoch als angeboren ansieht („Daß gleichwohl die Natur in uns

\footnotetext{
${ }^{80}$ Burke: The Sublime and the Beautiful, S. 87.

${ }^{81}$ Kant, Immanuel: Anthropologie in pragmatischer Hinsicht. In: Ders.: Schriften zur Anthropologie, Geschichtsphilosophie, Politik und Pädagogik, 2. Hg. von Wilhelm Weischedel. Frankfurt a. M. ${ }^{7} 1988$ (Werkausgabe, Bd. 12), S. 399-694, hier S. 580.

${ }^{82}$ Kant: Anthropologie, S. 580, Hervorheb. im Original.

${ }^{83}$ Kant: Anthropologie, S. 518.

${ }^{84}$ Vgl. Kant: Anthropologie, S. 584 f.

${ }^{85}$ Vgl. Kant: Anthropologie, S. 600.

${ }^{86}$ Kant: Anthropologie, S. 581.

${ }^{87}$ Kant: Anthropologie, S. 600.

${ }^{88}$ Kant knüpft mit seiner Affekt-Anthropologie hier an zeitgenössische Theorien an, die sich in der Dramentheorie ebenso wie in medizinischen Diskursen über Affektivität niederschlagen, vgl. Port: Pathosformeln, S. 59 ff., 68 ff. Port verweist auf das „Grundproblem des aufgeklärten Umgangs mit vehementen affektiven Impulsen“: „Affekte und Leidenschaften sind nicht primär Instrumente, sondern in erster Linie Gegenstände von Sorge, wenn es um besonnenes, tugendhaftes Verhalten zu tun ist" (S. 60).

${ }^{89}$ Kant: Anthropologie, S. 580, Hervorheb. im Original.

${ }^{90}$ Kant: Anthropologie, S. 580.
} 
die Anlagen dazu eingepflanzt hat ${ }^{(991}$ ), erteilt er ihnen aber eine Zweckmäßigkeit. Sie dienen laut seiner Anthropologie dazu,

provisorisch, ehe die Vernunft noch zu der gehörigen Stärke gelangt ist, den Zügel zu führen, nämlich den moralischen Triebfedern zum Guten noch die des pathologischen (sinnlichen) Anreizes, als einstweiliges Surrogat der Vernunft, zur Belebung beizufügen $\left[\ldots . .{ }^{92}\right.$

Emotionalität nimmt so bei Kant zumindest in der Ausprägung als Affekt die Rolle eines Stellvertreters der Vernunft ein, solange es an dieser (noch) mangelt. ${ }^{93}$ Der Affekt ist zudem insofern für Kant erträglich, als er eine Belebung des Willens durch die Verknüpfung der Ideen der Vernunft mit der für diese beispielhaften Anschauung darstellt; d. h. insofern die Vernunft den Affekt begründet, in Kants Worten „nicht als Wirkung, sondern als Ursache eines Affektes in Ansehung des Guten seelenbelebend"94 sei.

Die Problematik des „stärkeren sinnlichen Gefühl[s]“95 des Affektes als „,diejenige Bewegung des Gemüts, welche es unvermögend macht, frei Überlegungen der Grundsätze anzustellen, um sich darnach zu bestimmen", 96 findet sich bereits in Kants Analytik des Erhabenen. Kant sieht dort eine Ausnahme bei der Pathologisierung des Affekts nur für den Enthusiasmus gegeben, ,weil er eine Anspannung der Kräfte durch Ideen ist, welche dem Gemüt den Schwung geben, der weit mächtiger und dauerhafter wirkt, als der Antrieb durch Sinnesvorstellungen“. 97 Der Enthusiasmus bietet also jene Affizierung durch Vernunft, die Kant in seiner Anthropologie befürwortet. ${ }^{98}$

\footnotetext{
${ }^{91}$ Kant: Anthropologie, S. 582.

${ }^{92}$ Kant: Anthropologie, 582 f., Hervorheb. im Original.

${ }^{93}$ Damit steht Kant nicht allein da. Auch Herder propagiert den Vorschlag, Leidenschaften und Triebe als sinnliches Schema der in ihnen waltenden Vernunft zu begreifen. Siehe Herder, Johann Gottfried: Vom Erkennen und Empfinden der menschlichen Seele. Bemerkungen und Träume. Riga 1778; siehe auch Port: Pathosformeln, S. 66.

${ }^{94}$ Kant: Anthropologie, S. 583.

${ }^{95}$ Kant: Anthropologie, S. 583, Hervorheb. im Original.

${ }^{96}$ Kant, Immanuel: Analytik des Erhabenen. In: Ders.: Kritik der Urteilskraft. Hg. von Wilhelm Weischedel. Frankfurt a. M. ${ }^{12} 1992$ (Werkausgabe, Bd. 10), S. 164-276, hier S. 198.

${ }^{97}$ Kant: Analytik des Erhabenen, S. 199.

${ }^{98}$ Die evaluierende Differenzierung von Emotionen zeigt sich auch weiter bei Kants Unterscheidung zwischen wackeren Affekten und schmelzenden Affekten. Erstere schätzt er im Zusammenhang mit dem dynamisch-Erhabenen in ihrer Eigenschaft, das Bewusstsein unserer Kräfte, jeden Widerstand zu überwinden, wecken zu können. Die schmelzenden Affekte jedoch, welche die Bestrebungen zu widerstehen selbst zum Gegenstand der Unlust machen, ordnet er mit den dazugehörigen Rührungen dem Bereich der „Empfindelei“ zu. Vgl. Kant: Analytik des Erhabenen, S. 199. Zu Kants Einschränkungen in der Befürwortung des Enthusiasmus als Spielart des Erhabenen und zugleich zu tadelndem Affekt siehe Pries, Christine: Einleitung. In: Dies. (Hg.): Das Erhabene. Zwischen Grenzerfahrung und Größenwahn. Weinheim 1989, S. 1-30, hier S. 10 f. Zur Wandlung des Enthusiasmus von der affektuellen Erregung (bei Platon) über die
} 
Und doch setzt Kants Philosophie des Erhabenen das Gefühl zentral bei der, wie Jean-François Lyotard es in seinen Kant-Lektionen nennt, ,Montage ' der Bedingungen der Möglichkeit einer objektiven Erkenntnis überhaupt. ${ }^{99}$ Wie Lyotard herausarbeitet, lässt sich Kant hier an sich selbst vorführen, indem man das Gefühl des Erhabenen in seiner epistemischen Wirkungsweise nachvollzieht. Denn die Empfindung ${ }^{100}$ ist grundsätzlich notwendig für das Denken. Sie informiert das Gemüt über seinen Zustand:

\begin{abstract}
Man kann sagen, daß die Empfindung bereits ein unmittelbares Urteil ist - des Denkens über sich selbst. Auf der Grundlage seiner jeweiligen Tätigkeit urteilt das Denken, ob es sich ,gut' oder ,schlecht' fühlt. Dieses Denken synthetisiert also den Denkakt, der sich gerade anläßlich eines Gegenstandes vollzieht, mit dem Affekt, den ihm dieser Akt bereitet. Der Affekt ist der innere Widerhall des Akts, seine ,Reflexion‘.101
\end{abstract}

Die Empfindung ist für Lyotard bereits eine unmittelbare Vorstellung des Gegenstandes:

Jeder Denkakt wird also von einem Gefühl begleitet, das dem Denken seinen ,Zustand“ signalisiert. Doch dieser Zustand ist nichts anderes als das Gefühl, das ihn signalisiert. Über seinen Zustand informiert zu sein, bedeutet für das Denken, diesen Zustand zu empfinden, affiziert zu sein. Die Empfindung (oder das Gefühl) ist gleichzeitig der Zustand des Denkens und der Fingerzeig, der das Denken durch diesen Zustand über seinen Zustand unterrichtet. Das erste Charakteristikum der Reflexion besteht in der blitzartigen Unmittelbarkeit und der vollkommenen Koinzidenz von Fühlendem und Gefühltem, so daß sogar die Unterscheidung von aktiv und passiv bei diesem ,Fühlen' für das Gefühl unangemessen ist, weil sich damit Objektivität und mit ihr Erkenntnis abzuzeichnen begönne. ${ }^{102}$

Daher, so spitzt es María Peña Aguado in ihrer Überblicksdarstellung der $\ddot{\text { Asthe- }}$ tik des Erhabenen zu, hat das Gefühl des Erhabenen eine spezifische Verweisfunktion: „Das Erhabene ist kein Epitheton, sondern ein Zustand. Dieser Zustand ist allein als Gefühl erfahrbar. Dieses Gefühl tritt als Symptom auf."103 Dieses Symptom ist wiederum ein gemischtes Gefühl bestehend aus Lust und Unlust. Reflexion besteht nach Lyotard bereits darin, dass sich die Empfindung allein auf

gemütshafte Prägung im Kontext aufklärerischer Gefühlsethik bis zur transitorischen Stimmung (bei Heidegger) siehe Meyer-Sickendiek: Affektpoetik, S. 85 ff., bes. S. 95 f.

${ }^{99}$ Vgl. Lyotard: Die Analytik des Erhabenen, S. 19.

${ }^{100}$ Lyotard rückt den Begriff der Empfindung deutlicher in Richtung des Gefühls, als dies bei Kant der Fall ist. Vgl. den Eintrag ,Empfindung“ in: Eisler, Rudolf: Kant-Lexikon. Nachschlagewerk zu Kants sämtlichen Schriften, Briefen und Handschriftlichem Nachlass. Hildesheim 1961, S. $115 \mathrm{f}$.

${ }^{101}$ Lyotard: Die Analytik des Erhabenen, S. 20.

${ }^{102}$ Lyotard: Die Analytik des Erhabenen, S. 21.

${ }^{103}$ Peña Aguado, María Isabel: Ästhetik des Erhabenen. Burke, Kant, Adorno, Lyotard. Wien 1994, S. 13. 
dieses Unterscheidungskriterium Lust/Unlust bezieht: „Das für diesen Unterschied zuständige Gemütsvermögen ist das Gefühl der Lust und Unlust, dem auf der Seite der sogenannten Erkenntnisvermögen die bloße Urteilskraft entspricht" . ${ }^{104}$ Christine Pries hat dies, an Lyotard geschult und in Anlehnung an Kant, deutlicher in seiner Prozesshaftigkeit untersucht und unterscheidet zwei Phasen in der Erfahrung des Gefühls des Erhabenen. In der ersten Phase gelingt es der Einbildungskraft ,als sinnlichem und endlichem Vermögen“105 bei der Begegnung mit bestimmten Naturphänomenen, die über alle Maßen groß oder über alle Maßen mächtig zu sein scheinen, nicht mehr, ,die auf sie einstürmenden Eindrücke zu verarbeiten. [...] Die Einbildungskraft scheitert, der Übergang [vom Schönen zum Erhabenen] mißlingt; die Natur scheint zweckwidrig zu sein. Daraus ergibt sich Unlust." ${ }^{106}$ In einer zweiten Phase gesellt sich jedoch zu dieser Unlust trotzdem Lust,

denn in der weiteren Reflexion kommt dem betreffenden Subjekt der Gedanke, daß selbst die so übergroße und übermächtige Natur, gemessen am unendlichen Ideenvermögen (der Vernunft) nur veschwindend klein ist und ihre Macht zwar der endlichen, sinnlichen Seite des Menschen, nicht aber diesem seinen intelligiblen Vermögen etwas anhaben kann, daß es sich also als Vernunftswesen in Sicherheit befindet und der Natur überlegen ist. ${ }^{107}$

Pries generalisiert hier über Kants Unterscheidung in der Analytik des Erhabenen zwischen dem mathematisch-Erhabenem (dem über alle Maßen Großen) ${ }^{108}$ und dem dynamisch-Erhabenen (dem über alle Maßen Mächtigen) ${ }^{109}$ hinweg einen prozesshaften Begriff des Erhabenen. Im Hinblick auf die Naturerfahrungen und Tierbegegnungen in der primatologischen Feldforschung und der Möglichkeit affektiver Erkenntnis, die in der Folge am Text herausgearbeitet werden soll, ist es sinnvoll, auf diese Unterscheidung bei Kant noch einmal einzugehen. Denn die beiden Varianten des Erhabenen sind in der Analytik des Erhabenen bezogen auf zwei unterschiedliche Vermögen.

Das mathematisch-Erhabene, „, mit welchem in Vergleichung alles andere klein ist " ${ }^{110}$ betrifft die rohe, aber ungefährliche Natur, welche in der Anschauung ihrer Erscheinungen ,die Idee ihrer Unendlichkeit bei sich führt“. ${ }^{111}$ Deswegen verwendet die Einbildungskraft an ihr ,,ihr ganzes Vermögen der Zusammenfassung

\footnotetext{
${ }^{104}$ Lyotard: Die Analytik des Erhabenen, S. 22, Hervorheb. im Original.

${ }^{105}$ Pries: Einleitung, S. 9.

${ }^{106}$ Pries: Einleitung, S. 9.

${ }^{107}$ Pries: Einleitung, S. 9.

${ }^{108}$ Vgl. Kant: Analytik des Erhabenen, S. 169 ff.

${ }^{109}$ Vgl. Kant: Analytik des Erhabenen, S. 184 ff.

${ }^{110}$ Kant: Analytik des Erhabenen, S. 171, Hervorheb. im Original.

${ }^{111}$ Kant: Analytik des Erhabenen, S. 178.
} 
fruchtlos“112 und muss den Begriff der Natur auf ein „übersinnliches Substrat (welches ihr und zugleich unseren Vermögen zu denken zum Grunde liegt) führen“. ${ }^{113}$ Da dieses ,über allen Maßstab der Sinne groß ist", 114 werden der Einbildungskraft ,ihre Schranken und Unangemessenheiten, doch aber zugleich ihre Bestimmung zur Bewirkung der Angemessenheit mit derselben als einem Gesetze" "115 bewiesen. Kant schließt daraus:

\begin{abstract}
Also ist das Gefühl des Erhabenen in der Natur Achtung für unsere eigene Bestimmung, die wir einem Objekt der Natur durch eine gewisse Subreption (Verwechslung einer Achtung für das Objekt statt der für die Idee der Menschheit in unserm Subjekte) beweisen, welches uns die Überlegenheit der Vernunftbestimmung unserer Erkenntnisvermögen über das größte Vermögen der Sinnlichkeit gleichsam anschaulich macht. ${ }^{116}$
\end{abstract}

Das Gefühl des Erhabenen setzt sich hier zusammen aus einem Gefühl der Unlust, das aus der „Unangemessenheit der Einbildungskraft in der ästhetischen Größeneinschätzung “117 erwächst, und einer ,dabei zugleich erweckte[n] Lust“, die aus der „Übereinstimmung eben dieses Urteils der Unangemessenheit des größten sinnlichen Vermögens mit Vernunftideen, sofern die Bestrebung zu denselben doch für uns Gesetz ist", ${ }^{118}$ erfolgt. Dass wir also feststellen können, dass unsere Einbildungskraft zur ästhetischen Erfassung nicht ausreicht, erzeugt Lust, denn diese Feststellung selbst ist eine Äußerung der Vernunft, die wir so in uns gesetzmäßig tätig sehen. In seiner, mathematischen' Form ist das Erhabene somit:

ein Gefühl, daß wir reine selbständige Vernunft haben, [...] dessen Vorzüglichkeit durch nichts anschaulich gemacht werden kann, als durch die Unzulänglichkeit desjenigen Vermögens, welches in Darstellung der Größen (sinnlicher Gegenstände) selbst unbegrenzt ist $[\ldots] .{ }^{119}$

Das mathematisch-Erhabene betrifft also die Anschauung jener Natur oder ihrer Objekte, welche uns in ihrer unermesslichen Größe überwältigen und mit der Grenze unseres Erkenntnisvermögens zugleich auf dieses selbst verweisen. Pries schlägt daher vor, das Erhabene als Grenze zu definieren und als „Signatur unserer Zeit" ${ }^{* 120}$ zu lesen:

\footnotetext{
${ }^{112}$ Kant: Analytik des Erhabenen, S. 178.

${ }^{113}$ Kant: Analytik des Erhabenen, S. 178.

${ }^{114}$ Kant: Analytik des Erhabenen, S. 178.

${ }^{115}$ Kant: Analytik des Erhabenen, S. 180.

${ }^{116}$ Kant: Analytik des Erhabenen, S. 180.

${ }^{117}$ Kant: Analytik des Erhabenen, S. 180.

${ }^{118}$ Kant: Analytik des Erhabenen, S. 181.

${ }^{119}$ Kant: Analytik des Erhabenen, S. 182.

${ }^{120}$ Pries: Einleitung, S. 30.
} 
Das Erhabene ist das Gefühl einer Krise und hat [...] durchaus indikatorischen Wert. Es macht uns fühlen, was es heißt, just ,bis unter die oberste Schwelle' gekommen zu sein. Es bezeugt nicht mehr als das Bewußtsein dieser Grenze, aber auch nicht weniger. Es ist die Grenze. ${ }^{121}$

Das dynamisch-Erhabene, welches Kant in der Analytik des Erhabenen vom mathematisch-Erhabenen abgrenzt (jedoch weniger explizit ausführt), findet sich dagegen in derjenigen Natur, die furchterregend ist. Die furchterregende Natur aktiviert etwas anderes: Sie zeigt uns „unsere physische Ohnmacht" ${ }^{\text {“122 }}$ auf. ${ }^{123}$ Dabei erhöht sie laut Kant zugleich jedoch die Seelenstärke über ihr gewöhnliches Mittelmaß und lässt uns in uns „ein Vermögen zu widerstehen“ ${ }^{124}$ entdecken, „welches uns Mut macht, uns mit der scheinbaren Allgewalt der Natur messen zu können“ ${ }^{125}$ Im ästhetischen Urteil wird die Natur hier als erhaben beurteilt, „,weil sie unsere Kraft (die nicht Natur ist) in uns aufruft, um das, wofür wir besorgt sind [...], als klein, und daher ihre Macht [...] für uns [....] für keine solche Gewalt an[zu]sehen, unter die wir uns zu beugen hätten" ${ }^{126}$ Das dynamisch-Erhabene fördert somit in der Mischung aus Unlust an der Macht, die die furchterregende Natur auf unser Gemüt ausübt, und Lust daran, Widerständigkeit gegen die Gewalt der Natur über uns zu empfinden, eine Freiheitserfahrung zutage.

Das mathematisch-Erhabene bezieht sich auf das Erkenntnisvermögen und produziert eine Erfahrung der Vernunft anhand ihrer Begrenztheit, für die das Gefühl des Erhabenen das Symptom darstellt. Das dynamisch-Erhabene bezieht sich auf das Begehrungsvermögen und ermöglicht eine Freiheitserfahrung am Erlebnis der Widerständigkeit, dessen Äußerung ebenfalls das Gefühl des Erhabenen ist. Beide Formen des Erhabenen, so leitet Peña Aguado ab, zeigen letztlich in der Erhabenheit als „Denkungsart“ eine Subjektivität, „die uns erst durch das Gefühl des Erhabenen bewußt wird [...]. Es handelt sich um eine Selbsterkenntnis des Ich, das gleichzeitig Beobachter und Beobachtetes ist. " ${ }^{127}$ Die primatologischen Autor/

\footnotetext{
${ }^{121}$ Pries: Einleitung, S. 30, Hervorheb. im Original.

${ }^{122}$ Kant: Analytik des Erhabenen, S. 186.

${ }^{123}$ Dabei adaptiert Kant Edmund Burkes Kopplung des Erhabenen an das Schreckliche: „Whatever is fitted in any sort to excite the ideas of pain and danger, that is to say, whatever is in any sort terrible [...], is a source of the sublime; that is, it is productive of the strongest emotion which the mind is capable of feeling“. Burke: The Sublime and the Beautiful, S. 58, Hervorheb. im Original. Bei Burke ist die Ohnmacht jedoch nicht allein eine physische: „The passion caused by the great and sublime in nature [...] is astonishment; and astonishment is that state of the soul, in which all its motions are suspended, with some degree of horror. In this case the mind is so entirely filled with its object, that it cannot entertain any other, nor by consequence reason on that object which employs it. [...] No passion so effectually robs the mind of all its powers of acting and reasoning as fear. $[\ldots]$ Whatever therefore is terrible $[\ldots]$ is sublime too, whether this cause of terror, be endued with greatness of dimension or not" (S. 95 f.).

${ }^{124}$ Kant: Analytik des Erhabenen, S. 185.

${ }^{125}$ Kant: Analytik des Erhabenen, S. 185.

${ }^{126}$ Kant: Analytik des Erhabenen, S. 186.

${ }^{127}$ Peña Aguado: Ästhetik des Erhabenen, S. 52.
} 
innen als professionelle Beobachter/innen stoßen nun in der Feldforschung auf Situationen, in denen das Erhabene als Modell empfindungsbasierter Erkenntnis und vor allem dieser Art von affektiver Selbsterkenntnis anwendbar ist.

\subsubsection{Empfindsame Erkenntnisse des Reisenden: George Schallers The Year of the Gorilla (1964)}

Wie bereits angedeutet, stellt sich George Schaller ${ }^{128}$ mit seinem The Year of the Gorilla in eine Traditionslinie sowohl der Reiseliteratur als auch der Affenforschung: nicht nur, indem er die Explorer-Autoren nennt, die vor ihm Afrika und insbesondere die Virunga-Region bereist haben; sondern vor allem auch, weil er seinen Text abgrenzt von seiner ein Jahr zuvor erschienenen wissenschaftlichen Monographie The Mountain Gorilla. Nicht wie diese ,a compendium of facts, discussing the apes as subjects to be studied" (21), solle The Year of the Gorilla nun sein, sondern ein Text, der dem Emotionalen ausdrücklich Raum gebe, heißt es im Vorwort. Hier werden Affen besprochen ,,as acquaintances whose activities my wife and I discussed at the end of each day“; hier gibt es ,space to reveal the enjoyment I derived from roaming across grassy plains and uninhabited forests and climbing mist-shrouded mountains“ (21). Somit stellt Schaller sein emotionales Erleben und Beurteilen dezidiert in den Vordergrund und misst ihm eine Qualität und Bedeutung für die umfassende und ergiebige Darstellung seiner Erfahrung, über ein Jahr in Afrika Gorillas erforscht zu haben, bei. Der Text ist darin weitaus mehr einem Reise- als einem Forschungsbericht ähnlich. ${ }^{129}$

\footnotetext{
${ }^{128}$ George Schaller erforschte zunächst das Verhalten von Vögeln, bevor er von seinem Doktorvater John ,Doc' T. Emlen darauf hingewiesen wurde, dass die National Academy of Sciences eine Expedition zur Erforschung von Freiland-Gorillas plante. Nach ausgiebigem Studium der vorhandenen Literatur zum Gorilla und Beratung durch Clarence Ray Carpenter brach Schaller im Februar 1959 in Begleitung seiner Frau Kay zum damaligen Albert-Nationalpark (heute der ruandische Parc national des volcans und der kongolesische Virunga-Nationalpark) auf, um unter der Leitung Emlens Gorillas zu studieren. Nachdem Emlen im Juli 1959 abreiste, verbrachten die Schallers insgesamt ein Jahr in Kabara in der Virunga-Vulkanregion, um mehrere Gorilla-Gruppen zu beobachten, bis sie unter dem Druck der Dekolonisierung im September 1960 den Kongo verlassen mussten. 1963 kehrte Schaller noch einmal kurz zurück, um mit einem Fotografen des National Geographic Magazine Farbfotos der Gorillas zu erstellen, wendete sich dann später in seiner Karriere aber Löwen, Pandas und Antilopen zu.

${ }^{129}$ Eine Abgrenzung zum ,reinen ' Forschungsbericht ließe sich mit Bezug auf den empfindsamen Reisebericht vornehmen, der sich durch die überragende Stellung des Gefühlsmäßigen und einer Zersplitterung der Empirie auszeichnet, sowie einer Neigung, Wirklichkeit in Sinnbilder zu verwandeln, und sich anderen literarischen Formen zu öffnen, vgl. Brenner: Der Reisebericht, S. 194. Diese Entwicklung hin zu einer Poetisierung des Reiseberichts zeigt sich teilweise auch im Afrikareisebericht des die Gattung diversifizierenden 19. Jahrhunderts, auf den Schaller hauptsächlich rekurriert. Dafür gibt es durchaus faktischen Anlass: Wesentlich mehr als bei anderen Autor/innen des vorliegenden Korpus ist Schallers Forschungsarbeit zunächst eine ausgiebige Reisebewegung, bevor er sich nach Kabara aufmacht und hier mit dem längeren Forschungsaufenthalt teilsesshaft wird.
} 
In der Darstellung seiner Erlebnisse konzentriert sich Schaller auf die Wahrnehmung der Umgebung durch die Sinne (, senses") und die Beschreibung von Eindrücken durch Empfindungen (,feelings'). Das erste Kapitel in The Year of the Gorilla beginnt bereits mit dem Hinweis auf ,a special feeling of elation in having finally reached a destination that has occupied the mind for months, a destination that is completely outside one's experience“ (23). Schaller erfährt und schildert die Naturbegegnung vor allem als sinnliche Wahrnehmung, emotionale Erfahrung und spürbares Erlebnis. Bevorzugt verwendet er dabei neben der Empfindung die Erfahrung (,experience') als Begriff:

It is an exhilarating experience to wander alone through unknown forests when everything is still new and mysterious. The senses sharpen, bringing into quick focus all that is seen and heard. (38)

It was a wonderful feeling to sit near these animals and to record their actions as no one had ever done before. (44)

To see African wild life in all its abundance and variety, living as it always lived, was one of the most priceless experiences I have ever had. (71)

Die ungewohnte Umgebung führt bei Schaller zu einer Schärfung der Wahrnehmung, wie er bereits im ersten dieser Zitate deutlich macht. In der Abgeschiedenheit eines überwachsenen Tales findet er sich bspw. „wholly relaxed but receptive to any small adventure that might come my way" (97, Hervorheb. MS). An anderer Stelle geht es darum, die Gorillas jeden Tag wieder aufzuspüren: eine der Jagd nicht unähnliche Situation, ,the most tense and exciting part of the day“ (112). Hier schreibt Schaller: „For minutes I stood motionless, never so keyed to receive the slightest stimulus that even the distant whirr of a sunbird's wing was enough to startle me“ (112, Hervorheb. MS). Nicht nur die Sicht (,to scan the forest ahead for glimpses of a black body among the weeds", 112), auch der Geruchssinn (,The musty, somewhat sweet odour of gorilla hung in the air.“, 42) und vor allem das Gehör (,stopped, listened, and moved again“, 42; „The air was alive with sounds“, 55 ; ,listening intently for the snapping of a branch or the rumbling of a stomach“, 112) werden entscheidende Instrumente in der Dschungel-Umgebung.

In der auf das Sinnliche konzentrierten Darstellung finden sich Stimmungsbilder und Eindrücke für die afrikainteressierten Leser/innen wieder, wie sie aus dem Genre des Reisebericht bekannt sind: Das Unbekannte birgt für den Reisenden eine Fülle neuer Erfahrungen; die Aufgabe, als erster Weißer/Europäer/Forscher/ usw. an dieser Stelle dieses zu beobachten, lädt diese Erfahrung mit einer spürbaren Bedeutung auf. Die Fokussierung des Textes auf die empfindsame Eindrücklichkeit und die sinnliche Erfahrung zeigt jedoch auch eine Empfänglichkeit für die empfindungs- und affektbasierte Erkenntnis an. Dies wird vor allem dort explizit, wo Schaller über die Qualität der Empfindung als Instrumentarium sinniert:

On several occasions resting animals became uneasy while I was watching them from a distance. I was upwind, completely silent, and they obviously had not seen me. Yet they seemed to sense that something was not quite right. Perhaps they responded to subliminal stimuli, too vague to be assimilated consciously; or perhaps some other sense warned 
them of possible danger. I have had similar experiences when wandering through the forest. Suddenly I had the feeling, in fact, I knew, that gorillas were close by, yet I had neither seen, heard, nor smelt them. More often than not, I was correct. Most naturalists, I feel sure, have had similar encounters with animals. (114)

Schaller versucht hier zu ergründen, worin diese Art , sechster Sinn“ (,subliminal stimuli, too vague to be assimilated consciously“, „some other sense“) besteht und wie verlässlich er ist (,More often than not, I was correct“). Er stellt aber auch eine Verbindung her zwischen dem Spüren der Gorillas (,,to sense that something was not quite right") und einem aus der Empfindung - ohne bewusste sinnliche Grundlage - gewonnenen Bewusstsein des Forschers: „I had the feeling, in fact, I knew [...], yet I had neither seen, heard, nor smelt them." Bezeichnenderweise versucht er dieses für andere „naturalists“ zu generalisieren, in dem er eine Formulierung verwendet, die ebenfalls dem Bereich einer empfindungsbasierten Gewissheit entlehnt ist: „I feel sure“ (Hervorheb. MS). Man kann hieran ableiten, dass sich Schaller dem Gefühl des Erhabenen bereits grundsätzlich öffnet, indem er sein Wahrnehmungs- und Erklärungsrepertoire erweitert bzw. seiner Forschungssituation anpasst. Und tatsächlich lassen sich in The Year of the Gorilla zwei Passagen finden, die sich als Erfahrungen des Erhabenen untersuchen lassen: das Gefühl angesichts des Affen (ein affeninduziertes Erhabenes) und angesichts der gewalttätigen Natur (in einer genuinen Erfahrung des dynamisch-Erhabenen).

\subsubsection{Das affeninduzierte Erhabene}

Schaller und ,Doc" Emlen sind an den Hängen des Karisimbi unterwegs und haben bislang nur Nester und andere Hinterlassenschaften der Gorillas gefunden. Doch am dritten Tag, schreibt Schaller, „I heard a sound that electrified me - a rapid pok-pok-pok, the sound of a gorilla pounding its chest" (41, Hervorheb. im Original). Da Schaller das Tier nicht finden kann, machen sich Doc und er am nächsten Tag wieder in dieselbe Richtung auf und haben diesmal Erfolg. Die Gorillas sind tatsächlich in der Nähe:

Somewhere ahead and out of sight, a gorilla roared and roared again, иииа-иииа! an explosive, half-screaming sound shattered the stillness of the forest and made the hairs on my neck rise. I took a few steps and stopped, listened, and moved again. The only sound was the buzzing of insects. (41, Hervorheb. im Original)

Nach dieser Exposition folgt Schaller dem Geräusch und ,[f]inally I saw them, on the opposite slope about two hundred feet away, some sitting on the ground, others in trees“ (41 f.). Dies ist der erste vollständige Sichtkontakt und Schallers Blick fällt sogleich auf das - Du Chaillu und Akeley zufolge - paradigmatische Exemplar des Gorillas: „An adult male, easily recognizable by his huge size and grey back, sat among the herbs and vines. He watched me intently and then roared. [...] A few other animals moved around in the dense vegetation“ (42). Schallers ästhetisches Urteil betrifft zunächst die überraschende Schönheit der Tiere: 
Accustomed to the drab gorillas in zoos, with their pelage lustreless and scuffed by the cement floors of their cages, I was little prepared for the beauty of the beasts before me. The hair was not merely black, but a shining blue-black, and their black faces shone as if polished. (42)

Nach dieser alliterarischen und repetitiven Emphase (,,beauty of the beasts before me“ korrespondiert mit „black, [...] blue-black, [...] black“") ${ }^{130}$ tritt er in einen Rapport mit dem Silberrücken:

\begin{abstract}
We sat watching each other. The large male, more than the others, held my attention. $\mathrm{He}$ rose repeatedly on his short bowed legs to his full height, about six feet, whipped his arms up to beat a rapid tattoo on his bare chest, and sat down again. He was the most magnificent animal I had ever seen. His brow ridges overhung his eyes, and the crest of his crown resembled a hairy mitre; his mouth when he roared was cavernous, and the large canine teeth were covered with black tartar. He lay on the slope, propped up by his huge shaggy arms, and the muscles of his broad shoulders and silver back rippled. He gave an impression of dignity and restrained power, of absolute certainty in his majestic appearance. I felt a desire to communicate with him, to let him know by some small gesture that I intended no harm, that I wished only to be near him. Never before had I had this feeling on meeting an animal. As we watched each other across the valley, I wondered if he recognized the kinship that bound us. (42)
\end{abstract}

In der Klammer einer Interaktion des Einander-Beobachtens geschieht hier bei Schaller einiges, das sich mit Pries in Richtung des Erhabenen als Umgang mit einem Paradoxon lesen lässt. Für Christine Pries ist die Widersprüchlichkeit das Charakteristikum des Erhabenen, das „durch Gegensatzpaare beschrieben [wird], in deren Spannungsfeld es sich konstituiert." ${ }^{131}$ Bei George Schaller liegt der Widerspruch in dieser Passage zwischen den Hinweisen auf das (ästhetisch) Hässliche und der fast metaphysischen Größe des mathematisch-Erhabenen. Der Gorilla hat „,short, bowed legs“, Augenbrauenwülste (,brow ridges overhung his eyes"), sein Mund ist „cavernous“, mit Zähnen „,covered with black tartar“; er ist „,shaggy“. An anderer Stelle gibt Schaller an, Gorillas erweckten ,the superficial impression of slightly retarded persons with rather short legs, wrapped in fur coats" (216). Sie wirken wie ein Derivat des Menschlichen, eine Art missgebildete Imitation. Zugleich jedoch verleiht Schaller dem Gorilla-Silberrücken an dieser Stelle nicht nur die Pose eines klassischen Aktes („He lay on the slope, propped up on his [...] arms, the muscles of his broad shoulders and silver back rippled.") und die Charakteristika eines Ausstellungsstückes (,shiny blue-black“, „faces shone as

\footnotetext{
${ }^{130}$ Diese Konzentration auf das Schwarze des Gorillas scheint zunächst Burkes Gedanken zur ,Terrorhaftigkeit" und daher Eignung zum Erhabenen von Dunkelheit und Schwarzheit, auch bei „black bodies“, zu spiegeln. Burke erklärt diese Wirkung aus dem sensorischen Kontrast heraus: „Black will always have something melancholy in it, because the sensory will always find the change to it from other colours too violent". Burke: The Sublime and the Beautiful, S. $275 \mathrm{ff}$. Es ist jedoch auffallend, wie wenig Schaller den bei Du Chaillu noch so ausgiebig bedienten Topos des ,Schrecklichen Gorillas' bedienen möchte.

${ }^{131}$ Pries: Einleitung, S. 6.
} 
if polished“", Hervorheb. MS). Er nennt ihn auch „the most magnificent animal“". Der Gorilla hat nicht nur eine „crown“, die sogar dem Zeichen der Bischofswürde (,a hairy mitre“) ähnelt; Schaller sieht in ihm „dignity and restrained power“ und „absolute certainty in his majestic appearance“. 132

Der Widerspruch zwischen der deformierten menschlichen Ähnlichkeit und der majestätischen wenn nicht sogar metaphysisch besetzten Größe und Würde ruft in Schaller nicht nur Achtung hervor, wie es Kants Formel des Gefühls des Erhabenen bedingt. Schaller empfindet auch akut ein Begehren: „I felt a desire to communicate with him“. Dennoch handelt es sich an dieser Stelle um eine Version des mathematisch-Erhabenen, jenes Erhabenen also, das weniger das Begehrungs- als das Erkenntnisvermögen anspricht. Die Kommunikation dient der Vermittlung von Schallers Intentionen und Bedürfnissen (,to let him know by some small gesture that I intended no harm, that I wished only to be near him“") und sie resultiert in dem Gefühl einer Einsicht bzw. Erkenntnis, dass dieses Tier besonders ist: „Never before had I had this feeling on meeting an animal. [...] I wondered if he recognized the kinship that bound us" (Hervorheb. MS). Der letzte Satz fungiert dabei zugleich als eine Selbsterkenntnis - dieses Tier und ich sind verbunden - und als ein Erklärungsansatz für die Art von affektiver Wirkung - so fühle ich, weil dieses Tier und ich uns ähnlich sind. ${ }^{133}$

Das ästhetische Urteil basiert hier auf Empfindungen, die durch die komplexe Ähnlichkeit zwischen Mensch und Tier und deren paradoxe Wahrnehmung entstehen. Zunächst weiß die Einbildungskraft die widersprüchlichen und übermächtigen Eindrücke der ,Naturerscheinung Gorilla ${ }^{6134}$ nicht zu verarbeiten. Dann

\footnotetext{
${ }^{132}$ All dies sind seit Burke überlieferte Topoi des Erhabenen; vgl. „Magnificience“ und das erst in der zweiten Edition von Burkes Enquiry beigefügte Kapitel „Power“. Burke: The Sublime and the Beautiful, S. $140 \mathrm{ff}$. u. $110 \mathrm{ff}$.

${ }^{133}$ In dieser Passage echot dabei zum einen, ins Positive gewendet, das Schockmoment des ,kinship'-Diskurses des euroafrikanischen Kolonialismus, wie er sich exemplarisch in Joseph Conrads Heart of Darkness zeigt. Auch dort ist das Erkennen von ,kinship' mit affektiver Erkenntnis verbunden, so wenn der Binnenerzähler um seinen getöteten Steuermann trauert und ,the intimate profundity of that look he gave me when he received his hurt“ als ,a claim of distant kinship affirmed in a supreme moment" empfindet, oder die Indigenen im Herzen des Kontinents als „,not inhuman" begreifen und sich eingestehen muss, dass ,what thrilled you was just the thought of their humanity - like yours - the thought of your remote kinship with this wild and passionate uproar". Conrad, Joseph: Heart of Darkness. In: Paul B. Armstrong (Hg.): Heart of Darkness. Authoritative Text. Backgrounds and Contexts. Criticism. New York/London 2006, S. 3-77, hier S. 51 u. 36. Vgl. auch Lorenz, Matthias N.: Distant Kinship. Joseph Conrads „Heart of Darkness“ in der deutschen Literatur von Kafka bis Kracht. Stuttgart 2017, S. 29 ff. Zum anderen findet sich hier im Erkennen der Verwandtschaft im Blickkontakt auch schon der Moment einer möglichen radikalen Zäsur im anthropologischen Denken, das das Schwinden der Tier-Mensch-Differenz begreift. $\mathrm{Zu}$ den philosophischen Folgen einer derart auf das Posthumanistische verweisenden Zäsur siehe Derrida, Jacques: L'Animal que donc je suis. Hg. von Marie-Louise Mallet. Paris 2006.

${ }^{134} \mathrm{Ganz}$ im Sinne der Theoretiker des Erhabenen des 18. Jahrhunderts lässt sich der Gorilla in den Raum der Naturphänomene einordnen, die die Ästhetik des Erhabenen mit den geographischen und naturgeschichtlichen ,Entdeckungen“ der Neuzeit prägen. Vgl. auch Bühler, Benjamin: Ecocriticism. Eine Einführung. Stuttgart 2016, S. 100 ff.
} 
erfolgt jedoch eine Vernunftsleistung, die darin besteht, diesen Mangel durch Einordnung in ein Ähnlichkeits- als Verwandtschaftsverhältnis zu überkommen und sich damit das eigene intelligible Vermögen bewusst zu machen - etwa im Gegensatz zu dem des Gorillas, von dem Schaller später schreibt, seine „,very existence, free from want and free from problems" sei „mentally an evolutionary dead-end“ (225). In der Nähe der Tiere zu sein, ,,a wonderful feeling to sit near these animals and to record their actions as no one had ever done before" (44), wie er weiter schreibt, lässt sich so als Genuß des Gefühls des Erhabenen lesen, das durch eben diese Nähe hergestellt und damit im wörtlichen Sinne potenziell ,fassbar` wird.

\subsubsection{Das gewaltvolle Erhabene}

Das affeninduzierte Erhabene steht jedoch in Zusammenhang mit dem Einfall des Erhabenen dort, wo die Erfahrung stärker an dem von Kant abgeleiteten Modell der (über-)mächtigen Natur orientiert ist. Das Gorilla-Habitat ist für die Erfahrung des Gefühls des Erhabenen der Primatolog/innen so ausschlaggebend wie die Gorillas selbst. Im Folgenden soll daher ergänzend zum affeninduzierten Erhabenen analysiert werden, wie sich dieses gewaltvolle Erhabene im Angesicht der überwältigenden Natur bei Schaller gestaltet.

Schaller besteigt allein den Vulkan Nyiragongo. Dabei führt ihn sein Weg durch unwirtliches Gelände, ,through a maze of lava blocks strewn wildly about the slope“ (237), und er ist bereits affiziert von Ungeduld und Ungewissheit: „I quickened my pace, straining upward, not really knowing what lay ahead but impatient to find out“" (237). Oben angekommen schildert Schaller Folgendes:

\footnotetext{
Then I stood on the rim, without thought, looking at the tremendous hole in the earth that went down and down and at the white stream that mushroomed upward, and all around me was a sound like the growling of a monstrous dog. My mind refused to sort out the individual impressions. I slipped off my pack and sat on a boulder, gazing into the depths.

At my feet the sombre perpendicular walls dropped four hundred feet to a broad shelf, followed by another drop, and another and another. Some twelve hundred feet below me bubbled a lava lake, often obscured by the stream that burst from two large vents above it. The black surface of the lake heaved and bucked like a captive creature in a pit - a pit two-thirds of a mile in diameter. Jagged red fissures like wounds opened in the black pool and spurts of molten lava squirted into air. (237)
}

Auf die Ungeduld und Neugierde des Bergsteigers und Naturliebhabers folgt am Ziel angekommen zunächst eine Art Apathie dem Phänomen gegenüber. Reflexionslos, ,without thought“, steht er an diesem Ort. Seine Einbildungskraft verweigert sich gar: „My mind refused to sort out the individual impressions“ (Hervorheb. MS). Ihm bleibt zunächst nichts, als in das Phänomen hineinzublicken, ,gazing into the depths“, und sich so ihm auszusetzen. Und was sieht er dort? Ein fürchterliches Loch gigantischen Ausmaßes, eine auch durch Repetition ausgestaltete Unendlichkeit: „went down and down“, „dropped [...], followed by another drop, and another and another". Schaller greift zum Vokabular des Schrecklichen und Unheimlichen mit „tremendous“, „,sombre“, „obscured“ 
und „black surface“. ${ }^{35}$ Alles hier ist in Bewegung, es „,went down“, „muschroomed upward“, ,dropped“, ,,bubbled“, „,burst“, „,heaved and bucked“ und ,squirted“. Das, was er sieht, beschreibt er mit der Bildlichkeit von Höllendarstellungen: alptraumhafte Monster (, a sound like the growling of a monstrous dog“"), eingesperrte Kreaturen (,like a captive creature in a pit"), klaffende Wunden (,[j]agged red fissures like wounds“").

Wie um diesen affektiven Eindruck von der gewalttätigen, schrecklichen Natur zu anästhetisieren, reagiert Schaller mit der Information für die Lesenden, mit dem Nyiragongo habe er nun insgesamt alle acht Virunga-Vulkane bestiegen, „to my knowledge the second person to have done so“ (237). Auf die Erfahrung der Ohnmacht angesichts der überwältigenden Natur in Form des Vulkankraters, angedeutet in Reflexionslosigkeit und körperlicher Schwäche, reagiert Schaller mit einer Selbstermächtigung durch Anerkennung der vollbrachten Leistung. Dann jedoch verraten seine Tätigkeiten wieder die körperliche Wirkung des Blicks in den Abgrund. Schaller „,crawled“ bereits ,,[a]t dusk“ und ,,weary“ in seinen Schlafsack und „dozed“ (237). Es folgt der zweite Teil der Erfahrung:

I awoke after dark. A soft red glow suffused the sky as I crawled on all fours to the crater rim. I lay there and stared at a sight so beautiful that my heart wanted to cry out and waves of shivers moved over my back. Far below, the lake of lava shone bright orange-red, and as the glow rose, and spread outward it turned a delicate purple. I was an intruder, spying under the cover of darkness into a purple vortex at the centre of the earth lying before me naked, its emotions laid bare. (237)

Wieder ist es ihm unter dem Eindruck des Naturphänomens nur möglich, sich kriechend, „on all fours“, zu bewegen und am Kraterrand zu liegen. Doch nun hat sich das Bild, das Schaller schildert, wesentlich verändert: Jetzt herrscht ein „soft glow“ am Himmel und im Krater (,as the glow rose“). Der Lavasee ist nicht mehr schwarz und kreatürlich, sondern schillert in warmen Farben: ,,shone bright orange-red, and [...] turned a delicate purple." Was er sieht, affiziert ihn direkt körperlich: ,a sight so beautiful, that my heart wanted to cry out and waves of shivers moved over my back“. ${ }^{136}$ Dies ist verbunden mit der Einsicht, ein Eindringling zu sein, ein Uneingeweihter, der das Geheimnis schaut: ,an intruder,

\footnotetext{
${ }^{135}$ Auch hier wieder ruft Schaller burkesche Topoi des Erhabenen ab: Burke betont unter dem Stichwort „Vastness“ die Tiefe gegenüber der Länge oder Höhe, sieht die Wahrnehmung von „Infinity“ als „source of the sublime“, ebenso „Obscurity“ und, wie bereits erwähnt, „Darkness“. Burke: The Sublime and the Beautiful, S. $127 \mathrm{ff}$.

${ }^{136}$ Wenn Peña Aguado das Erhabene als Zustand beschreibt, der allein als Gefühl erfahrbar sei, so fügt sie an, dieses Gefühl trete auch als somatisches Symptom auf: „Stotternde Wörter, zitterndes Schweigen, ein fast unvernehmbares Murmeln sind Zeichen, welche eine besondere Sensibilität erwecken. Diese Empfänglichkeit kann nur erreichen, wer in der Lage ist, in den Widersprüchen, Paradoxien und Antagonismen die Wege zu einem Anderen aufzuspüren“. Peña Aguado: Ästhetik des Erhabenen, S. 13.
} 
spying under the cover of darkness into a purple vortex at the center of the earth". In aufschlussreicher Prosopopöie findet Schaller dieses Geheimnis „lying before me naked, its emotions laid bare."

Letztlich ist es der Forscher selbst, der hier nackt und emotional dem Naturphänomen als Übung des Erhabenen ausgesetzt erscheint. Zitternd kriecht er wieder zurück zu seinem Lager und wendet nun den Blick gen Himmel (,gazed at the vault of heaven"). Dies beschreibt er poetisch insinuierend - wieder ist es der „gaze“ und nicht der ,look'; für den Himmel verwendet er die religiöse und poetische Vokabel „heaven“ statt „,sky“; und er wählt den vieldeutigen Term „vault“, der sowohl Kuppel als auch Gruft bedeuten kann. Hier geht die Erfahrung nun in einen dritten Teil über:

Soon an icy wind tore at my body and moaned among the boulders and hollows. A full moon rose and poured its silver light over the bleak slopes. It sailed nearer and nearer, the black depths behind it growing more and more distinct. Across the valley rose Mt. Mikeno, dark and still, to the stars. I was alone in space, adrift in the sky. Never before had the insignificance of my being struck me so overwhelmingly. On one side a fiery red glow suffused my body, attesting that the earth is not a stable abiding place; on the other, the moon caressed me with a soft light from the immensity of the cosmos. (237 f.)

Schallers Blick fällt angesichts der empfundenen Ohnmacht auf sich selbst und seine Rolle zwischen dem Himmelsgewölbe und dem Vulkankrater. Die Einsamkeit dieser physisch spürbaren und affektiv fühlbaren Erfahrung (,,alone in space, adrift in the sky“) wird hier zur überwältigenden Einsicht in die eigene Bedeutungslosigkeit - „the insignificance of my being struck me overwhelmingly“ - und Ausgesetztheit angesichts des gewaltvollen Naturschauspiels von Himmel und Erde in ihrer Weite und Rohheit.

Diese ganze Passage verweist in der allein durch die Anschauungskräfte des Individuums erfolgenden Verwandlung der höllenartigen, furchterregenden in die schmerzhaft schöne, berührende Natur auf die Prozesshaftigkeit des dynamisch-Erhabenen. Peña Aguado fasst dies zusammen als „für das Gefühl des Erhabenen charakteristische Rührung", 137 die der Bewegung folge, die in unserem Gemüt hervorgerufen werde. Die dadurch erregte Lust ergibt sich ,aus einem Moment der Leere, der Furcht und Angst“: 138 „Es handelt sich um eine ,negative Lust', bei der das Spielerische, das die Schönheit bezeichnet, sich in eine Ernsthaftigkeit verwandelt, als Folge dieses ersten Moments der Berührung, in der unsere Lebens- und Erkenntniskräfte gehemmt und gelähmt werden." ${ }^{139}$ Bei Schaller findet sich eine solche ,negative Lust" durchaus in der Widerständigkeit, die das Subjekt in seiner Bedeutungslosigkeit und Ausgesetztheit doch am Rande des Kraters in den Himmel blicken und den furchterregenden, aber nicht gefürchteten Moment genießen lässt: erfüllt vom roten Schein des Kraters,

\footnotetext{
${ }^{137}$ Peña Aguado: Ästhetik des Erhabenen, S. 51.

${ }^{138}$ Peña Aguado: Ästhetik des Erhabenen, S. 51.

${ }^{139}$ Peña Aguado: Ästhetik des Erhabenen, S. 51.
} 
,attesting that the earth is not a stable abiding place“ und gekost vom sanften Licht des Mondes „from the immensity of the cosmos“ (238).

\subsubsection{Erhabene Forschung und die Erkenntnis des Spürbaren: Dian Fosseys Gorillas in the Mist (1983)}

George Schallers Empfänglichkeit für Phänomene des Erhabenen und mit ihnen für eine affektive Erkenntnis (sei dies jene von der Verwandtschaft zum Forschungstier, die Fremdheit des Menschen als ,Intruder' in der Natur oder die Bedeutungslosigkeit und zugleich Einbettung der eigenen Existenz) speist sich zu nicht geringem Teil aus seiner Offenheit dem Genre des Reiseberichts und seinen Vorläufern in der empfindsamen Beschreibung der Natur gegenüber. Dian Fossey dagegen nennt ihr publikumswirksames Buch zwar Gorillas in the Mist und bedient damit im Titel eine gewisse Afrikaromantik. Sie hält sich in ihren Schilderungen jedoch trotz der Heterogenität der Textform überwiegend von Elementen des sentimentalen Reiseberichts fern und wirkt sogar mit ihren zeitgenössischen wissenschaftlichen Publikationen entnommenen Datenpassagen und Tabellen einer ,Zersplitterung der Empirie“ (Brenner) entgegen. ${ }^{140}$ Vielleicht ist es gerade dem Bemühen um eine möglichst breite Datensammlung zu verdanken, dass sich auch in Gorillas in the Mist eine Ausrichtung auf das Sinnliche als Instrumentarium des Naturstudiums zeigt. ${ }^{141}$ In ihrer Wiedergabe der ersten Begegnung mit einem Gorilla 1963 in Kabara konzentriert sich Dian Fossey auf eine rhetorisch verstärkte Abfolge sinnlicher Eindrücke: ${ }^{142}$

Sound preceded sight. Odor preceded sound by an overwhelming musky-barnyard, humanlike scent. The air was suddenly rent by a high-pitched series of screams followed by the rhythmic rondo of sharp pok-pok chestbeats from a great silverback male obscured behind what seemed an impenetrable wall of vegetation. (3)

Auch später verweist sie dezidiert vor allem auf den Geruchssinn als wichtiges Instrument, wenn sie sich dem ,pungent body odor, resembling human nondeodorized

\footnotetext{
${ }^{140}$ Vgl. Brenner: Der Reisebericht, S. 194.

${ }^{141}$ Diese Wissensgenerierung aus dem Sinnlichen lässt sich wieder bis zu Burke zurückverfolgen: „[W]here there is a clear concurrence of all [senses], we may with more certainty speak of any one of them. By this means, they bear witness to each other; nature is, as it were, scrutinized; and we report nothing from her, but what we receive from her own information". Burke: The Sublime and the Beautiful, S. 237, Hervorheb. MS.

${ }^{142}$ Fossey reist zunächst 1963 auf eigene Kosten zu einer siebenwöchigen Safari nach Ostafrika und kann in diesem Rahmen nicht nur die paläontologische Ausgrabungsstätte von Mary und Louis Leakey aufsuchen, sondern auch auf den Spuren von George Schaller, ,[a]n eminent American scientist", und Carl Akeley, ,an American naturalist who had been responsible for urging the Belgian government to create the Albert National Parc for the protection of the mountain gorillas" (GM, S. 2), Joan und Alan Root begleiten, die in den Virungas Gorillas fotografieren (S. 1-18).
} 
sweat smell“, „odiferous deposits“, „olfactory clues“ oder „,most outstanding odor“ (45) widmet. Die Gorilla-Geräusche werden ebenfalls hervorgehoben: Neben dem typischen männlichen Gebrüll erwähnt sie „soft purring sounds“ und ,stomach rumbling“ (53) als ,disyllabic belch volcalizations“ (53), welche sie sogar imitiert und $\mathrm{zu}$ einem Forschungsfokus macht. ${ }^{143}$ Ebenso nennt sie die „technique“, aufmerksam geradeaus zu blicken: „much circuitious tracking time could be saved by looking ahead of a group's trail for signs of disturbance“ (43). „Much can be gained by crawling, rather than walking, along gorilla trail“" (45), schreibt Fossey und übertrifft dabei die Schärfung der sinnlichen Wahrnehmung, wie sie bereits bei Schaller auffällt, noch durch die Aufgabe des aufrechten Ganges zugunsten eines Ertastens, Erriechens, und Erfühlens der Welt der Gorillas (und anderer nicht-menschlicher Tiere): „I feel with my fingertips for the deep imprints“ (44), schreibt Fossey und findet sich ,belly-crawling under a long dark tunnel“ (44).

Wie Schaller vor ihr erfasst sie die Qualität des Erfolgs, den sie darin hat, die Tiere aufzuspüren und durch die Imitation ihrer Laute an sich zu gewöhnen, als Gefühl: „It is an extraordinary feeling to be able to sit in the middle of a resting group of gorillas and contribute to a contented chorus of belch vocalizers" (54). Fossey ist jedoch in ihrem Programm der sinnlichen Annäherung an die Gorillas und Gewöhnung der Tiere an ihre Präsenz erfolgreicher als Schaller. Ihr Gefühl angesichts des Affen manifestiert sich nicht mehr 200 Fuß von diesen entfernt, sondern inmitten der Gorillagruppe. Dian Fossey beschreibt stärker als Schaller die Wirkung der Gorillas auf sie selbst in einem Vokabular emotionaler Ergriffenheit, das den Affekt auch als Übergriffiges konzipiert. ${ }^{144}$ Sie ist „struck by the physical magnificence of the huge jet-black bodies blended against the green palette wash of thick forest foliage“ (3, Hervorheb. MS). Individualität und Schüchternheit der Gorillas werden als ,the most captivating impression“ (4, Hervorheb. MS) wahrgenommen. Dort, wo sie Emotionen klar benennt, sind ihre Eindrücke dem Bereich starker Affiziertheit entliehen:

The sense of exhilaration I felt when viewing the heartland of the Virungas for the first time from those distant heights is as vivid now as though it had occurred only a short time ago. (18, Hervorheb. MS)

I longed for the joyful sense of exhilaration I had always felt when beginning to climb to Kabara. (22, Hervorheb. MS)

Since that day I never have had the slightest difficulty in recalling the elation felt upon being able to renew my research with the mountain gorilla. (25, Hervorheb. MS)

\footnotetext{
${ }^{143}$ Siehe Fossey, Dian: Vocalizations of the mountain Gorilla (Gorilla gorilla beringei). In: Animal Behaviour 20 (1972), S. 36-53.

${ }^{144}$ Damit folgt sie der lateinischen Wurzel des Begriffes Affekt: afficere als „hinzutun, einwirken, antun, anregen“. Im Gegensatz zum konkurrierenden griechischen pathos (,,allgemein für Empfindung, Gefühl und speziell für Schmerz, Krankheit, Leiden“) wird der Affekt als etwas begriffen, „was dem Menschen angetan wird, was er durch seine Abhängigkeit von der Außenwelt ,erleidet ““. Grimm: Affekt, S. 19.
} 
As a pioneer I sometimes did endure lonelines, but I have reaped tremendous satisfaction that followers will never be able to know. (25, Hervorheb. MS)

Wie sich hier zeigt, sind diese Emotionen - freudige Erregung, ein Hochgefühl, immense Befriedigung - meist verbunden mit den ,Früchten der Wissenschaft', wie die Ernte-Referenz (,reaped“) im letzten Zitat andeutet, oder der Aktivität des Forschens selbst. So ist sie „thrilled to observe“ (31) oder fühlt Euphorie „upon being able to renew my research" wie im dritten obigen Zitat. Zudem werden diese Emotionen der Leserschaft mit Nachdruck präsentiert, indem sie temporal reflexiv erscheinen: ,as vivid now as though it had occurred only a short time ago“, „I had always felt", „Since that day I never have had“ oder ,that followers will never be able“, ganz im Sinne einer zusätzlichen Affiziertheit durch die Erinnerung: „Recounting the history of Group 5, I find myself somewhat overwhelmed by the mosaic of memories - humorous, perplexing, sad, tender, loving“" (105).

Dian Fossey zeigt sich ebenfalls offen für ein affektives Erleben und emotionales Wahrnehmen im Forschungskontext - nur fasst sie die emotionalen Qualitäten der Feldforschung noch pointierter zusammen als Schaller dies in seinem Erinnerungsbuch tut:

Personally, I found the explorations throughout the volcanoes some of my most memorable forest experiences - the challenge of the search, the thrill of encountering a new gorilla group, the awesome beauty of the mountains revealed by virtually every turn in a trail, and the pleasure of making a ,home " with only a tent and the benevolence of nature. (158)

Trotz der distanzierenden Raffung und Abstraktion der Emotionalität finden sich bei Fossey ebenfalls Szenen, in denen das Erhabene einfällt. Eine Passage insbesondere bildet dabei ein Äquivalent zu Schallers affeninduziertem Erhabenen. Fossey berichtet von einem späten Nachmittag im Jahr 1977, an dem sie eine der ihr vertrauten Gruppen beobachtet hat:

Thinking the gorillas had gone, I slowly stood up to determine their direction for the next day's contact. Suddenly I heard a noise in the foliage by my side and looked directly into the beautifully trusting face of Macho, who stood gazing up at me. She had left the group to come to me. On perceiving the softness, tranquility, and trust conveyed by Macho's eyes, I was overwhelmed by the extraordinary depth of our rapport. The poignancy of her gift will never diminish. (200 f.)

Auf das Erschrecken angesichts des eigenen Irrtums und der unerwarteten physischen Nähe des Gorilla-Weibchens folgt die Anschauung (,the beautifully trusting face of Macho"), die Reflexion (,She had left the group to come to me.") und die Einordnung des Verhaltens des Gorillaweibchens in eine Ordnung der emotionalen Nähe (,the softness, tranquility, and trust conveyed by Macho's eyes"). Dieses Erschauen äußert sich symptomatisch in einem Gefühl der Überwältigung, das Fosseys Emotionalität semantisch bereits ein Moment der Erfahrung des Erhabenen einschreibt: „I was overwhelmed“. Schallers „,kinship that bound us“ $(Y G, 42)$ ist hier eine „extraordinary depth of our rapport“ (GM, 201), eine Ver- 
schiebung von der Einordnung in ein taxonomisches Verwandtschaftsverhältnis zu einer außerordentlichen tiefen Verbindung, die auf Austausch beruht. ${ }^{145}$ Damit verleiht Fossey dem Gefühl eine nachwirkende Empfindungsqualität: einer andauernden Schärfe des Eindrucks, der ,poignancy“ von Machos ,Geschenk“

Es gibt eine zweite Passage, in der das Erhabene geltend gemacht werden kann, doch sie ist komplexer aufgebaut als bei Schaller. Auf ihrer Safari 1963 macht Fossey laut Gorillas in the Mist eine sehr positive Initiationserfahrung. Hier ist vieles ,extraordinary“ (3) und „most captivating“ (4) und Fossey beteuert „I shall never forget" (3) und „I left Kabara [...] with never a doubt that I would, somehow, return“ (4). Sie kehrt 1967 nach der Einwerbung von Leakeys Unterstützung und Forschungsmitteln nach Kabara zurück - wird jedoch schon nach kurzer Zeit aufgrund von politischen Wirren im Grenzgebiet bedroht, gefangen gesetzt und außer Landes geschafft ( $G M, 14$ ff.). Daraufhin fällt es Fossey zunächst schwer, in den Virungas auf der ruandischen Seite, die ihr offen stehen, eine ähnliche Erfahrung zu machen wie zuvor im Kongo. Beim Aufstieg auf den Visoke vergleicht sie die dicht besiedelten Hänge und das Eingreifen menschlichen Lebens in die Natur mit der durch menschliche Lebenszeichen weitgehend unberührten Erinnerung an Kabara. Bezeichnenderweise beginnt das zweite Kapitel unter dem Titel ,Second Beginning“ mit dem Satz „Ruanda is one of the most densely populated countries in the world“"(19). Bei ihrem ersten Afrika-Aufenthalt ist sie allein mit einem Fahrer unterwegs; der Aufstieg auf den Visoke beginnt nun dagegen mit einem ,long scraggy train of porters“ (21). Es geht durch ,a narrow maze of trails across cultivated fields“ (21) und Fossey stellt fest: „People seemed to be everywhere, a marked contrast to heading up to my first camp from the small Congo village of Kibumba and immediately plunging into the utter stillness of the dark forest below Kabara“ (21). Es regnet, sie friert, man läuft lange durch die Kultivationen und sieht nur ein paar Bäume: ,the only remnants of what once had been a magnificent forest. I longed for the joyful sense of exhilaration I had always felt when beginning to climb Kabara. The ascending route here was more like walking through a bombsite in the aftermath of a war" (22, Hervorheb. MS). Ehemals dem Naturpark zugehörige Gebiete sind hier ,,doomed to be culled for pyrethrum and people“ (22, Hervorheb. MS). Sie stellt fest „I am grateful that I knew this region even as late as 1967, for it will never be the same“ (22, Hervorheb. MS).

Die endzeitliche Stimmung, die sich hier in den Kriegsschauplatz-, Keulungs- und „never the same“-Anleihen sedimentiert, prägt die gesamte Passage bis zu diesem Punkt. Dann aber verändert sich die Natur in einem von Elephanten geschaffenen Vegetationstunnel für Fossey - durch frische Fäkalien: „Once within the thick bamboo, I felt a small bit of the magic of the wilderness when fresh

\footnotetext{
${ }^{145}$ Der Oxford English Dictionary definiert rapport als ,,[m]utual understanding between persons; sympathy, empathy, connection; a relationship characterized by these“ und ,a close and harmonious relationship in which the people or groups concerned understand each other's feelings or ideas and communicate well“. Siehe rapport, n. In: OED Online (2018), http://www.oed.com/ view/Entry/158.209 (13.03.2018).
} 
elephant droppings were sighted, as well as signs of gorilla“"(22). Auch hier bricht das Endzeitliche noch ein, wenn es heißt, dass

[t]en years later, when most of the elephants of the parklands had been killed by poachers, the sides of the tunnel were to become covered with thick layers of moss, obliterating for ever signs of one of the many animal species among the original inhabitants of the Virunga Volcanoes. (22)

Diese Zone ist jedoch bedeutsam für Fossey, sie ist ein ,dramatic entrance into the world of the gorilla“, ein ,passageway between civilization and the silent world of the forest" (22).

Es folgen über eineinhalb Seiten Informationen über die Vegetation und ihren Nutzen für die Gorillas. Dann kehrt Fossey wieder zu ihrer eigenen Empfindungswelt und zum physischen Erleben zurück und befindet die Strecke für steil und das Atmen für schwer. Sie ist „,delighted when the porter wanted to stop for a rest and a smoke“ (24). Der Ort für diese Pause ist besonders gut gewählt:

The spot they chose was a small meadow clearing where elephant and buffalo droppings had accumulated around a stream running through the middle of the clearing. The air was pure elixir and the running water refreshingly sweet and cold. The heavy fog and drizzle were just beginning to give way to the promise of welcomed sun. For the first time I could fully appreciate the extent of the herbaceous foliage that abounded on Visoke's steeper slopes to the north side of our trail. The terrain seemed to be very promising gorilla country. I grew tremendously eager to discover what lay ahead west of us, deeper within the heartland of the Virungas. (24)

Dieser Ort ist für Fossey ein ,locus amoenus', eine Lichtung inmitten des dichten Waldes (,herbaceous foliage“), mit Luft als „,pure elixir“", einem „,refreshingly sweet and cold" Strom und ,the promise of welcomed sun“. ${ }^{146}$ Nach dem Weg durch die „,dramatic entrance“ und den ,passageway“ ist dies der Umschlagplatz für ihre Stimmung: Hier, an diesem idyllischen Ort, zu Fosseys Freude ausgiebig bekotet durch Elefanten und Büffel, empfindet sie die Situation als ,,very promising“ und wird ,tremendously eager“". Alles wird hier idyllischer, aber auch größer und beeindruckender:

Distributed throughout the meadows, like so many powerful sentries, stood magnificent Hagenia trees, bearded by long lacy strands of lichen flowing from their orchid-laden limbs. The entire scene was backlit by sunlight, giving all a spectacular dimension no camera could record or eye believe. I have yet to see a more impressive spot in all of the Virungas or a more ideal location for gorilla research. (24 f., Hervorheb. im Original)

In dieser „scene“, gewinnt Fossey durch die ästhetische Erfahrung des idyllischen, aber durchaus durch die Baum-Wächter (,,like so many powerful sentries“)

\footnotetext{
${ }^{146}$ Ernst Robert Curtius definiert den ,locus amoenus“ als Lustort, der „ein schöner, beschatteter Naturausschnitt [ist]. Sein Minimum an Ausstattung besteht aus einem Baum (oder mehreren Bäumen), einer Wiese und einem Quell oder Bach“. Curtius, Ernst Robert: Europäische Literatur und lateinisches Mittelalter. Tübingen 1993, S. 202.
} 
mit den Insignien des Überwältigenden behafteten und dank der ungreifbaren Beleuchtung („,backlit by sunlight, giving a spectacular dimension no camera could record or eye believe") sich der Vorstellungskraft entziehenden Ortes die Einsicht, bleiben und forschen zu können, genau an dieser Stelle.

Fossey liegt aber das schwärmerische Genießen des Gefühls des Erhabenen nicht, der direkt anschließende Satz ist dokumentarisch gehalten und verschließt den Fokus auf das emotionale Erleben: „Exactly at 4:30 P.M. on September 24, 1967, I established the Karisoke Research Centre“ (25). Fossey setzt die aus dem Gefühl gewonnene Erkenntnis in ihrem Text direkt um in nüchterne Arbeitsschritte. Der ",next logical step“ ist die „selection of a Rwandese camp staff“ (25). Der nächste Absatz beginnt mit der bereits zitierten Fokussierung des Emotionalen auf die Forschung: ,Since that day I never have had the slightest difficulty in recalling the elation felt upon being able to renew my research with the mountain gorilla" (25, Hervorheb. MS). Fossey bedient sich in ihren Schilderungen der proleptischen und analeptischen Einschübe, um ihrer affektiv hergeleiteten Entscheidung Bedeutung und Rechtfertigung zu verleihen:

Little did I know then that by setting up two small tents in the wilderness of the Virungas I had launched the beginnings of what was to become an internationally renowned research station eventually to be utilized by students and scientists from many countries. As a pioneer I sometimes did endure loneliness, but I have reaped a tremendous satisfaction that followers will never be able to know. (25)

Die Symptome des Gefühls des Erhabenen sind in Fosseys Text nicht derart transparent und nachvollziehbar dargestellt wie bei George Schaller. Doch die Auswahl ihres Forschungsortes, ,what was to become an internationally renowned research station eventually utilized by students and scientists from many countries", beruht auf der ästhetischen Erfahrung - und einem an der Erfahrung der erhabenen Natur gefällten ästhetischen Urteil. Wie Schaller lässt sich Fossey auf eine Schärfung der sinnlichen Wahrnehmung und die Konzentration auf die Sinnenwelt ein. ${ }^{147}$ Enthält Schallers The Year of the Gorilla jedoch ganze Seiten der Ausführungen über die heimische Idylle mit seiner Frau Kay in Kabera, sogar Passagen aus deren Briefen an Familie und Freunde, die seine individuelle, außerordentliche Erfahrung des Gefühls des Erhabenen mit der familiären Heimeligkeit eines traditionellen Ehemodells kontrastieren, ${ }^{148}$ verwendet Fossey den Verweis auf die Nachteile

\footnotetext{
${ }^{147}$ An einer Betonung der Bedeutung von sinnlichen Wahrnehmungen für die Gewinnung von Wissen setzt auch die feministisch-epistemologische Betrachtung an, die sich der körperlichen Erfahrung und ihrem ontologischen Wert in den autobiographischen Forschungsnarrativen der Primatologinnen widmet. Siehe Bishop: Writing the Body Wild.

${ }^{148}$ Vgl. YG, S. 107 ff., 153 ff., 165 ff. Kay Schaller ,remained at Kabera, filling her time with sewing, baking, and all the other tasks of a housewife" (S. 153). George Schaller zitiert sie u. a. mit folgendem Bericht: „By about four o'clock I could begin to expect George home. Every so often I stepped oustide the cabin to look towards the trail. Finally he came, chilled and hungry,
} 
der Pionierarbeit (,,[a]s a pioneer") und gesteht Einsamkeit ein, um deutlich zu machen, welche Leistung sie vollbracht und welche Art von Belohnung sie dafür erhalten hat: Die ,tremendous satisfaction that followers will never be able to know" wird dabei zum Anklang an Kants Enthusiasmus. Bei Fossey wird Kants aus der Vernunft geborener Affekt allerdings zu einer aus der affektinduzierenden, ästhetischen Erfahrung des ,loecus amoenus“ entstehenden ,vernünftigen “ Entscheidung - die in der Formulierung in Gorillas in the Mist ihrerseits einen affektiven Zustand provoziert (,tremendous satisfaction“").

\subsubsection{Emotionalität und Paranoia: Robert Sapolskys $A$ Primate's Memoir (2001)}

Die Gefahr dieser Art von Affekt zeigt sich u. a. darin, welches ,Image“ die öffentliche Person Dian Fossey bis über den Tod hinaus begleitet. Robert Sapolsky beschreibt Fossey als „clearly the stuff of legends“, welche ,did little science of note beyond observing amazing things by sheer dint of her persistence, was openly contemptuous of most scientists doing fieldwork, and clearly wanted little more than to be a gorilla herself" (222). Trotz dieses harschen Urteils spielt die Primatologin für Sapolsky eine einzigartige Rolle. Sechs Monate nachdem Dian Fossey in ihrer Forschungsstation ermordet worden ist, reist der Neurobiologe, der zu dieser Zeit in Kenia Paviane im Feld erforscht, nach Karisoke. ${ }^{149}$

Sapolsky verbindet in A Primate's Memoir am Ende des kurzen Kapitels „The Mound Behind the 7-Eleven“ (219 ff.) das Interesse an Gorillas mit der emotionalen (und auch wissenschaftlichen) Unreife seiner Jugendzeit:

\footnotetext{
My scientific interests had not yet shifted from gorillas to baboons, and gorillas still resonated emotionally with me in an extraordinary way; during intermittent periods of depression that plagued me, I was dreaming more about gorillas than about humans. Thus, it was not surprising that Fossey was one of the humans I most admired. (223, Hervorheb. MS)
}

Sein Interesse an den Gorillas speist sich aus der Berichterstattung von und über Dian Fossey, ihrer kulturellen Resonanz, Publikumswirksamkeit ${ }^{150}$ und

\footnotetext{
and I would give him hot cocoa or bouillon, and if I had baked he was given an ,extra'. Then while he got into a warm sweater and dry pants, I hung his sodden clothes by the fire and sat beside him to hear about how the gorillas had been and what he had learned that day" (S. 157).

${ }^{149}$ Der Biologe und Neuroendokrinologe Robert Sapolsky verbrachte Ende der 1970er Jahre eineinhalb Jahre im Feld, um das Sozialverhalten von Pavianen, insbesondere deren Stressverhalten, zu studieren. In den folgenden 25 Jahren reiste er jedes Jahr wieder für einen kürzeren Zeitraum zur selben Paviangruppe und sammelt weitere Daten. A Primate's Memoir schildert raffend die Erlebnisse aus dieser langen Zeitspanne der Feldforschung. Vgl. auch Abschn. 4.3.

${ }^{150}$ So verweist Sapolsky auf das Gedicht The Observer von Adrienne Rich über Fossey, das sich an seiner Wand befunden habe. Vgl. PM, S. 223; Rich, Adrienne: Collected Poems 19502012. New York/London 2016, S. 264.
} 
Persönlichkeit. Fossey wird von ihm als sture, irrationale, emotionale Verweigererin gezeichnet (222 f.), und die Begegnung des enthusiastischen Studenten Sapolsky mit der desinteressierten Vortragenden Fossey in Cambridge fällt ernüchternd aus. Zugleich muss er hinsichtlich ihrer Auseinandersetzungen mit Wilderern im Bemühen um den Schutz der Gorillas anerkennen: „Undeniably, a more stable, rational person would have dealt with the situation in a less inflammatory way, but a more stable, rational one would never have been there to witness what was happening“" (224).

Nach diesem Persönlichkeitsprofil im Vorlauf schildert Sapolsky die eigene Reise zu den Berggorillas. Sie ist ihm möglich, da er nun ,that maturational stage“ (225) erreicht habe, ein Stadium, das sich auch durch die ökonomischen Mittel auszeichne, die eigenen Jugendideale vor Ort überprüfen zu können. Wie Schaller ( $Y G, 238$ ff.) und Fossey (GM, 20 f.) erwähnt auch Sapolsky die ethnischen Spannungen zwischen Hutu und Tutsi und die dichte Besiedlung des Landes $(P M, 225 \mathrm{f}$.), und auch bei ihm wird die Vegetation beschrieben, um einen landschaftlichen Eindruck des Aufstiegs zu geben. In der für seinen Text typischen ironisch-distanzierten Art versucht Sapolsky jedoch hier, die Szenerie nicht in die Kategorie der empfindsamen Naturbetrachtung fallen zu lassen:

Bamboo everywhere, moss-covered hagenia trees that have always looked silly to me unless they are shrouded in mist. Higher, onto a saddle of one of the volcanoes, a view of forest ahead of us, a small lake, fields of bushes. Onward, the rangers macheteing a way through fields of stinging nettles. Clouds and mist and chills and heat, somehow all simultaneously. Sweating and shivering. Sliding down a deep ravine, clambering the way back up the other side, more nettles, more bamboo. (226)

Durch Repetitionen und Ellipsen wird dabei eine emotionale Einfärbung vermieden, dafür jedoch stellt sich eine physische Symptomatik ein: Es entsteht der Eindruck der Atemlosigkeit und Nähe zum Geschehen. Hier wird die Verknappung der Syntax zum Symptom der Erschöpfung und Anspannung:

\footnotetext{
Another hour. Misty rain, but somehow warmer. More nettles. Something resembling a real path and a flattened clump of grass to the left of it. Large, fibrous, shredded turds in the middle, the type you would expect from a pro football player gone vegetarian. The gorillas. Fresh, last night's nest. Pushing ahead, tired and excited and impatient. Down another ravine $[\ldots]$. We stop, silent, willing to invent the sound to convince ourselves that they are close, and suddenly, we hear the unmistakable murmur, deep throaty, slow-motion, paternal. We rush/tiptoe up the other side and, on top of the ridge, I see my first wild mountain gorillas. (226)
}

Impressionistische Fetzen unterstützen das erzählerische Präsens in seinem Tempo, welches jedoch punktuell entschleunigt wird durch die humoristischen Vergleiche (,turds [...], the type you would expect from a pro football player gone vegetarian“). Mit dieser Widersprüchlichkeit korrespondiert auch die paradoxe affektive Mischung, die hier vorliegt: ,tired and excited and impatient.“

Aus dem bislang die Perspektive bestimmenden „we“ der Reisenden, die die Erfahrung des Aufstiegs - auch in ihren physischen Auswirkungen - teilen, wird 
bei der Sicht auf den ersten Gorilla das „I“ des empfindsamen Subjekts. Die folgende Passage kennt nur noch die Wahrnehmung des Ichs gegenüber dem ,they“ der Gorillas. Dieses Ich öffnet, wie schon Schaller und Fossey es taten, den Gorillas und ihrem Habitat gegenüber seine Sinne. Nach dem ersten Anblick beobachtet Sapolsky und lauscht dem Spiel der halbwüchsigen Gorillamännchen (,they’d pant“, „to catch their breath“, ,pound its chest“, 226). Besonders der Geruch der Gorillas wird hier wieder hervorgehoben: Er ist „comforting, musty, damp [...] like opening a trunk from the mildewed corners of a cellar that contains forgotten beloved objects“ (227). Dieser Implikation der Wiederbegegnung mit dem längst Bekannten einer vergessenen Kindheitserinnerung folgt ein Überwältigungsmoment:

I had a flood of thoughts and feelings. At the first sight, I thought, Now my eyes will well up with tears, but I was too intent on watching for that to happen. I wondered what my social rank would be if I had wound up a mountain gorilla. I was mesmerized by their eyes: their faces seemed less emotionally expressive than those of chimps or even baboons, but their eyes, you wanted to go swimming in. I tried not to make eye contact, not only because it's bad field technique and discomforts primates, but because the act would make me want to confess to unlikely crimes. I found myself with the barely controllable urge to scream, or to gibber dangerously among them, or to rudely kiss one, so that they would stomp me to death then and there and stop my suspense. I thought, They are far less socially interactive than baboons, they're kind of boring in fact - thank god I didn't come to study them, I'd be a fourteenth-year grad student by now. And at the same time, I thought I never wanted to budge from my spot. (227)

Die Erfahrung der Überwältigung als hereinbrechende innere Flut, die Gleichzeitigkeit von ,thoughts and feelings“, die Rührung in der Affiziertheit, und zugleich der Bann (,too intent on watching“, „,mesmerized by their eyes“, „I never wanted to budge from my spot"), sich nicht aus dem Anblick der Gorilla-Augen lösen zu können, erinnern an die körperliche Symptomatik des Erhabenen, wie sie Peña Aguado zusammenfasst: ${ }^{151}$ Vom Bedürfnis zu Schreien (,,barely controllable urge to scream“) über das unverständliche Stottern (,to gibber dangerously among them") und das Zuwendungsbedürfnis (,too rudely kiss one“) bis hin zum Todeswunsch (,that they would stomp me to death“) verweisen Sapolskys Empfindungen auf das Gefühl des Erhabenen. Und aus dieser affektiv erlebten Überwältigungserfahrung erwächst dabei eine Erkenntnis über das hier überpräsente „I“: Sapolsky macht sich Gedanken über die soziale Hierarchie der Gorillas und seine Rolle darin, er reflektiert die lange zuvor getroffene Entscheidung für Paviane als Untersuchungsgegenstand als Segnung (,thank god“).

Um seine Gefühle besser zu umschreiben, lässt Sapolsky apologetisch einen Traum folgen, ,that summarized my feelings far better than I could when awake“ (227), ,a dream so tender, so ludicrously sentimental, so full of beliefs that I do

${ }^{151}$ Vgl. Peña Aguado: Ästhetik des Erhabenen, S. 13 sowie Anm. 63 in Abschn. 2.3. 
not have when awake, that I still marvel at it" (227). In seinem so vom Vernunftsvermögen des Wachzustands bis hin zum Wunderbaren abgegrenzten Traum erscheint Folgendes:

God and angels and seraphs and devils all existed, in a very literal way, each with potential strengths and frailties much like our own. And I dreamt that the rain forests of the Mountains of the Moon were where god places the occasional angel born with Down's syndrome. (227)

Sapolsky referiert an dieser Stelle nicht explizit George Schaller. Dennoch sieht man dessen ,slightly retarded persons with short legs“ $(Y G, 216)$ in interessanter Koinzidenz Engeln mit einer sehr menschlichen Genmutation gegenübergesetzt. Sapolskys Traumwelt überführt die (evolutionäre) Mangelhaftigkeit, die Schaller den Gorillas attestiert, in eine theologische Skizze, in der Gorillas als auf ihre Weise gefallene Engel aufgehoben erscheinen. Genetik und Theologie vermählen sich hier zu einer eigenen Ordnung, in der die Gorillas ihren festen und sinnhaften Platz in ihrem Habitat zugewiesen bekommen. ${ }^{152}$

Diese erste Versenkungserfahrung, ausgelöst durch den begehrten und doch widerstrebenden Blick in die Augen der Gorillas (,their eyes, you wanted to go swimming in“, „I tried not to make eye contact, [...] because the act would make me want to confess to unlikely crimes"), geht über in das Schauen eines Gewaltigen und der damit einhergehenden Ohnmacht des Subjektes. Sapolsky bleibt noch eine Woche, sieht sich mehrfach die Gorillas an, wird aber immer deprimierter: „The gorillas were wondrous, but the weight of what was gone, removed, unmentioned, unanswered, irrevocable, became heavier" (227). Als Sapolsky auf den Karisimbi steigt, sieht er, wie weit der Mensch bereits in das kleine Habitat der Berggorillas eingedrungen ist: ,a world with no room for rain forests or the moon's mountains, a conspiracy to forget them altogether" (228). Der Einbuch des menschlichen Lebens in das Habitat der Gorillas, welcher von Fossey laut Gorillas in the Mist 1967 noch als Hindernis am Umschlagplatz der Lichtung überkommen werden konnte, ist nun, 1986, omnipräsent. Die Hilflosigkeit angesichts dieser unaufhaltsamen Bedrohung für die wenigen Tiere in diesem Gebiet manifestiert sich für Sapolsky bei einem Ausflug auf den Karisimbi physisch und psychisch: „It was on the top of that mountain that the week finally got to me and I had a night of African paranoia“" (228). Vordergründig ist die darauf folgende längere Textstelle , afrikanischer Paranoia ' ein ebenfalls wieder ironisch ausgestalteter Wettstreit mit einem widerwillig ihn begleitenden Park Ranger. Doch dieses ,sullen, sloe-eyed kid, with a face like a mask and a tense air of violence about him“

\footnotetext{
${ }^{152}$ Das Wunder(n) als „quintessential human response“ auf ,first encounters“ und seine Verwendung als Modus der Einsicht in Differenz in Diskursen der Entdeckung hat bereits Greenblatt diskutiert, siehe Greenblatt: Marvelous Possessions, S. 20. Sapolskys Traum dient vor diesem Hintergrund als Be- bzw. Verarbeitungsmodus des Wunderbaren, als eine Art ,containment'-Strategie, die das Außerordentliche in eine durch Genetik und Theologie doppelt sanktionierte Ordnung überführt und das Wunder derart abzubauen versucht.
} 
(228), das seinen Dienst am Touristen möglichst schnell und unkooperativ zu verrichten versucht und von Sapolsky mehrfach mit genuiner Antipathie belegt wird, kann für Robert Sapolskys Erfahrung auf dem Karisimbi kaum allein verantwortlich sein. ${ }^{153}$ Nicht nur ist hier der Druck der Woche durch die Höhe auch physisch spürbar (,I felt an edge of altitude sickness, my vision got blurry, my chest throbbed“, 228; „Your eyes throb, your balls throb, your head hurts constantly, your chest aches with each breath, everything is straining“, „my eyeballs throbbing 110 times a minute“, 229) und seine Gedanken kreisen um die Gorillas (,I mostly thought about gorillas“, 229), Sapolsky erleidet auch einen Angstanfall:

[I]t occurred to me for the first time to become afraid. Not only was Fossey murdered just six months before on this mountain [...], but tonight was almost certainly my night to get it. This may now sound facetious or exaggerated or farcical or just paranoid, but I was suddenly terribly frightened. I was alone on some volcano in a Central African country where I didn't know a soul, shut in this hut in the middle of an ice storm with a ranger, and I felt sure now the rangers had killed Fossey. As I reviewed the day, the week, my every word and action now seemed to have sealed my doom, to have convinced the watching rangers that I must be killed. I was genuinely frightened, near to panic. I desperately wanted to escape. I struggled to control my breathing, thought to cry out for help. I lay awake most of the night, with my pocketknife open at my side, and truly thought I was going to die. [...] At dawn, I felt foolish and angry and relieved, and I felt I had been lucky this time. (229)

Der Ausflug zu den Gorillas mit den körperlichen Strapazen der Bergbesteigung lässt Sapolsky nah an der Grenze der psychischen Gesundheit tänzeln. Retrospektiv ordnet er diese Passage in den Bereich der Groteske oder Farce ein (,facetious or exaggerated or farcical"). Doch das mindert die Empfindungsqualität und -quantität vor Ort nicht: Das Psychogramm beinhaltet entsetzliche Furcht und Panik (,I was suddenly terribly frightened“, ,genuinly frightened, near to panic“), ein Gefühl der Einsamkeit und Verlassenheit („I was alone“, „I didn’t know a soul“), der Eingeschlossenheit und Unausweichlichkeit (,,shut in“, ,,sealed my doom“), Fluchtgedanken (,wanted to escape“), Kontrollverlust und Atemnot (,struggled to control my breathing“), das Bedürfnis zu schreien (,wanted to cry out for help“), Schlaflosigkeit (,lay awake most of the night“), und Todesgedanken (,thought I was going to die“").

Für Kant ist in der Analytik des Erhabenen diejenige Natur (dynamisch-) erhaben, die als Macht keine Gewalt über uns hat. Sie ist furchterregend, aber um sie als Erhabenes beurteilen zu können, darf man sich selbst nicht fürchten: „Wer sich fürchtet, kann über das Erhabene der Natur gar nicht urteilen [...].

\footnotetext{
${ }^{153}$ „I had noticed him and already had a dislike for him“; „,my dislike for my guide began to build“; ,,[m]y dislike simmered“ (PM, S. 228); „There was not even a hint of the mythic camaraderie that enemies are said to feel for each other in a tough battle" (S. 229). Diese Art von direkter Ablehnung überrascht im Kontext von Sapolskys humoristisch aufbereiteten Forschungsmemoiren, in denen selbst zwielichtige Gestalten, die ihn mehrere Tage festhalten, wohlwollend ausgestaltet werden. Vgl. das Kapitel „The Coca-Cola Devil“ (S. 57-64).
} 
Jener fliehet den Anblick eines Gegenstandes, der ihm Scheu einjagt; und es ist unmöglich, an einem Schrecken, der ernstlich gemeint wäre, Wohlgefallen zu finden“. ${ }^{154}$ Während George Schaller den Vulkan, der durchaus eine reale Gefahr für sein Leben darstellen könnte, in seiner Erhabenheit erschauen und aus dem Gefühl des Erhabenen eine Selbsterkenntnis ziehen kann, ist Sapolsky durch den Affekt der panikartigen Furcht gebannt. Der Gegenstand, den er schaut, ist eine Natur, die furchterregend wird, weil sie nicht mehr, rein ' ist: topologisch weder unbesiedelt und unbedrängt noch historisch unbelastet durch den Menschen. Die Wirkung dieses Gegenstandes auf Sapolsky ist der obigen Schilderung nach von immensem Ausmaß, doch sie resultiert nicht in einem klassischen Gefühl des Erhabenen. Seine erschreckende Affiziertheit endet in der Feststellung, ,foolish, angry and relieved", sowie schlicht davon gekommen zu sein. Selbst als er auf der Bergspitze steht, sieht er nur mehr das Gewesene und Verlorene: „I looked out over Rwanda, Uganda, Zaire, and tried to imagine that it was once all rain forest full with gorillas“ (230). Der Versuch bleibt vergeblich. Hier zeigt sich ex negativo, was Pries betont: dass es im (klassischen) Gefühl des Erhabenen um die „Balance zwischen der ästhetischen Wahrnehmung und der rationalen Reflexion geht. “155 Sapolsky wird überwältigt von der Wahrnehmung eines immensen und übersubjektiven Verlustes; hier gibt es keine Möglichkeit der Selbst-Bestätigung durch das Vernunft- oder Begehrungsvermögen, beide scheitern vor dem Phänomen der vom Verschwinden bedrohten Natur. Insofern wirkt diese Erfahrung in A Primate's Memoir in Richtung von Christine Pries' Vorschlag, das Erhabene nicht länger als „Erhebung oder Überheblichkeit des Menschen zu verstehen“", ${ }^{2}$ sondern als „,die zutiefst kritische Situation eines ,Zuviel', eines Zuviel an Information, [...] einer Überwältigung und des Bewußtseins der Endlichkeit des Menschen." "157 Und diese Endlichkeit des Menschen wird von den Affenforscher/innen zunehmend in dem wahrgenommen, was der Mensch (be-)endet. In dieser Form scheint das Erhabene nicht länger als Zeichen der Dominanz des Subjektes. Stattdessen wirkt es als postmodernes, postsubjektives Erhabenes als Merkmal eines Mangels und ist damit eher dem 21. Jahrhundert, aus dem heraus Robert Sapolsky seine Forschungsmemoiren schreibt, angemessen. ${ }^{158}$

Letztlich lässt sich Robert Sapolskys Erfahrung auf den Spuren Fosseys so auch nicht als Scheitern einer Suche nach affektiver Erkenntnis beurteilen, wie

\footnotetext{
${ }^{154}$ Kant: Analytik des Erhabenen, S. 185.

${ }^{155}$ Pries: Einleitung, S. 29, Hervorheb. im Original.

${ }^{156}$ Pries: Einleitung, S. 28, Hervorheb. im Original.

${ }^{157}$ Pries: Einleitung, S. 29.

${ }^{158}$ Vgl. Peña Aguado: Ästhetik des Erhabenen, S. 96. Es lässt sich aber argumentieren, dass das 21. Jahrhundert in seiner anthropozänen Produktion des Mangels (an Habitat, an Natur- und Artenvielfalt, aber auch an Ressourcen) die Konsequenz jener Dominanz des Subjektes ist. Vgl. auch Abschn. 3.4.3.
} 
der Angstanfall zunächst nahe legen würde. Ganz im Gegenteil, so kann man mit Sianne Ngai argumentieren, lassen sich ,ugly feelings“ wie die Paranoia als ,feelings amoral and noncathartic, offering no satisfaction of virtue, however oblique, nor any therapeutic or purifying release " ${ }^{159}$ als diagnostische Instrumente und „models of subjectivity, collectivity, and agency“160 im Kontext einer politischen Ästhetik verstehen. Nicht nur erfährt Sapolsky wie George Schaller und Dian Fossey vor ihm das affeninduzierte Erhabene im Blick der Berggorillas und gewinnt angesichts des bedrohten Zustands der Natur ihres Habitats eine Einsicht in die Erscheinungsformen des Anthropozäns: ${ }^{161}$ Die ruandischen Virungas sind verändert durch Menschenhand, der Blickkontakt mit den Berggorillas wirkt als Mahnzeichen für ihr Verschwinden. An ,the graves of Fossey and the other primates“ (230), auf die er eher zufällig stößt, erfährt Sapolsky, wie seine Apostrophe an Fossey zeigt, darüber hinaus eine besondere Regung:

Fossey, you pain-in-the-ass saint, I do not believe in prayers or souls, but I will pray for your soul, I will remember you for all of my days, in gratitude for that moment by the graves when all I felt was the pure, cleansing sadness of returning home and finding nothing but ghosts. (230)

Die reine, reinigende Traurigkeit ist für Sapolsky in dieser Textstelle emotionaler Indikator für das Ende einer biographischen Epoche. Dieser Satz beendet die „Tenuous Adulthood“, den dritten Teil seines Buches A Primate’s Memoir. Somit steht die Fossey/Gorilla-Passage innerhalb des Textes nicht nur strategisch als Markstein am Ende eines biographischen Weges. Sie endet eben auch wieder mit einem Gefühl als Symptom einer empfindungsbasierten Erkenntnis: Für Robert Sapolsky wird durch die ,Rückkehr' zu den Gorillas als Totemtieren eines empfindsamen Jugendinteresses und zu dem Ort von Fosseys Feldforschung und Niedergang der Prozess einer Ablösung enthüllt. Sapolsky ist ganz Pavian-Forscher; durch die affektiven Abläufe und Einsichten, denen er an diesem Ort und angesichts der Affen ausgesetzt ist, erkennt er zum einen die Vergeblichkeit des Kampfes für ein Überleben der Gorillas rein als ästhetische Reflexionsmedien , unserer Verwandtschaft" und, mit Kant, die Achtung für seine eigene Bestimmung als neuroendokrinologischer Erforscher der (nicht vom Aussterben bedrohten) Paviane.

\footnotetext{
${ }^{159}$ Ngai, Sianne: Ugly Feelings. Cambridge, Mass. 2007, S. 6.

${ }^{160}$ Ngai: Ugly Feelings, S. 5.

${ }^{161}$ Zum Begriff des Anthropozäns als geologische Ära der Erdgeschichte, in der die globalen Auswirkungen der Menschheit einer ,Naturkraft‘ gleichkommen, siehe Steffen, Will/Grinevald, Jacques/McNeill, John: The Anthropocene: Conceptual and Historical Perspectives. In: Philosophical Transactions of the Royal Society A 369 (2011), S. 842-867 und Horn, Eva: Jenseits der Kindeskinder. Nachhaltigkeit im Anthropozän. In: Merkur 71/814 (2017), S. 5-17.
} 


\subsubsection{Das Erhabene als Egozentrik: Lukas Bärfuss' Hundert Tage (2008)}

Das Gefühl des Erhabenen am Gorilla in den Virungas verändert sich über die hier betrachtete Literatur hinweg: von Schallers an das klassische Modell des dynamisch-Erhabenen anknüpfender Überwältigungserfahrung am Vulkan und spezifisch andere Einsicht produzierenden affeninduziertem Erhabenen im Blick des Gorillas, über den Einfall gerade dieses Erhabenen in Fosseys eigene Emotionspolitik und Thematisierung empfindungsbasierter Erkenntnis für die Forschungsarbeit bis zu Sapolskys kritischem postmodernen Erhabenen. Das Gefühl des Erhabenen wirkt, so zeigt Sapolskys A Primate's Memoir, noch immer als nach innen gewendete empfindungs- und affektbasierte Erkenntnis, stößt aber in seiner Prozesshaftigkeit auf Schwierigkeiten, Lust und Unlust zu verbinden, weil der Mensch sich in die überwältigende Natur als noch gewaltigere und gewalttätigere Macht eingeschrieben hat (oder sie schlicht als Macht verdrängt hat). Diese anthropozäne Wendung in der Erfahrung des Erhabenen bildet auch der Roman Hundert Tage des Schweizer Schriftstellers Lukas Bärfuss in einer Passage zum Gorilla-Tourismus in Ruanda prägnant ab: Die Textstelle zeigt auf, wie das Erhabene anscheinend immer noch greift, jedoch nur mehr als eine konsumierbare, subjektbezogene spirituelle Erfahrung gelesen werden kann: „Einen Eingeweihten, der gerade die Bergregenwälder besucht hatte, erkannte man [...] auf den ersten Blick am seligen Lächeln, den glänzenden Augen, einer allgemeinen Wendung nach innen“ (145). Auch bei Bärfuss berichten diese Eingeweihten von der durch den Blick strukturierten Einsicht, vom affeninduzierten Erhabenen, das sich hier in jener Anerkennung durch das Angeschautwerden qua Gorilla ereignet:

$\mathrm{Ja}$, ich habe sie gesehen, murmelten sie entrückt, aber das ist nicht wichtig. Was ist denn wichtig, stieß man vielleicht nach, und dann erntete man einen Blick, in dem die Verachtung und das Mitleid zu erkennen waren, weil man selbst zu den Uneingeweihten gehörte. Sie haben mich gesehen, antworteten sie, ins Innerste meiner Seele haben sie geschaut. (145 f., Hervorheb. im Original)

Wie sich hier zeigt, deutet Bärfuss' Binnenerzähler David Hohl die Begegnung mit den Gorillas als eine Kult-artige Erfahrung mit „Eingeweihten“ (145, 156) und „Uneingeweihten“ (146), in der die Gorillas „die spirituellen Führer, so etwas wie der Nullmeridian, die Koordinate, auf die sich alle bezogen“ (146), sind. Auch David Hohl steigt hinauf zu den Gorillas und beschreibt seine Erfahrung in einer Szene ähnlich intensiv und sinnlich geprägt, wie dies auch die primatologischen Texte tun (,ein betörender Geruch“, „Licht durch die Nebelschwaden“, „Ich habe nie wieder etwas Bunteres gesehen“, „ein Grün in millionenfacher Schattierung“, 148). Obwohl ihm die Gorillas zunächst in einer Reprise des Deformations- bzw. Mutationsgedankens bei Schaller und Sapolsky „schwarzhaarige Trolle“ (148) sind, rutscht auch David unweigerlich in die Erfahrung des Erhabenen hinein, die hier deutlich als religiöses Erlebnis ausgedeutet wird: „Ich weiß nicht, wie lange es dauerte, bis auch ich bekehrt war" (148, Hervorheb. MS). Auch hier ist es der Blick eines Gorillas, der die Symptomatik des Erhabenen hervorruft: „Da drehte sich der 
Silberrücken müde nach mir um, und für eine winzige Sekunde trafen sich unsere Blicke“ (148). David steht „wie festgefroren da“ und will zunächst die affektive Überwältigung fliehen (,und mir überlegte, wie ich schnell und unauffällig verschwinden konnte" 149). Hier schlägt die Unlust zunächst in einem Erkenntnismoment in eine Form von Lust um: „Dann aber bemerkte ich, dass ich nichts weniger wollte als das, dass ich nicht aus Angst wie angewurzelt stehen geblieben war, sondern aus Liebe, aus Liebe zu dieser Kreatur, zu dieser Ruhe“ (149).

Gleichwohl wird in dieser Passage bei Bärfuss deutlich, dass das Erhabene postmodern ethisch nicht mehr haltbar sein kann, inmitten von Gewalt, Vergewaltigung und Genozid. Die Erörterung des spirituellen Wertes des Affen kippt subtil um in die Ökologie der ihn umgebenden Gewalt. So wiegt ein Gorilla ,zehn, hundert, sogar tausend Menschenleben“ (146) auf und die Kinder am Wasserloch, die David noch kurz zuvor mit dem Gorilla gemeinsam beobachtet hat, sind verschwunden, erwartet von jemandem, ,der Knüppel und Macheten mitgebracht hatte, dazu Schnüre“ (149). Die nachfolgende Passage fasst die Problematik des affeninduzierten Erhabenen in der anthropozänen Postmoderne zusammen. Zwar bezeichnet sich David als von ,der Gegenwart dieses Buddhas, dieses Bergmenschen, verzaubert[]“ und glaubt nicht mehr, „,dass es eine gute Idee der Evolution gewesen war, uns von den Bäumen herunterzuholen, und dass wir besser geblieben wären, was wir gewesen waren, wenn wir dafür diese Ruhe, Gelassenheit, diese Versenkung in den Moment hätten zurückgewinnen können“ (149). Aber der Preis der Evolution ist hier dezidierter, ,in dieser Angst leben“ zu müssen, ,die auch diese Kinder befallen haben muss, als sie den Männern gegenüberstanden, Gestalten mit dem ewigen, lächelnden Gesicht des Bösen, das schlecht war, weil es die wahre Absicht verbarg und die Furcht der Kinder zu zerstreuen suchte“ (149). Die Evolutionsgeschichte ist für den vom Genozid traumatisierten und desillusionierten David Hohl eine Teleologie des Bösen und der Verstellung. Der Gorilla ist in der Erfahrung des Erhabenen der Schlüssel zur schreckensinduzierten Erkenntnis darüber, was der Mensch durch seine Evolution hat auf sich nehmen müssen: mit der „verfeinerten Mimik“ „die Vernebelung unserer wahren Absichten“, die „Falschheit, Betrug, Täuschung“ (149) sind und bei Bärfuss den Menschen von der „Schöpfung“ (149) trennen. ${ }^{162}$ Konsequent wird der Abstieg vom Berg, der Abschied von den Gorillas als ,den Weisen vom Berg“ (150), kontrastiert mit Mord und Vergewaltigung. Es liegt Schuld darin, dass der Erzähler noch seine Erhabenheitserfahrung genießt, während die Kinder vergewaltigt und ermordet werden: ,[W]ährend ich beseelt war von der Begegnung

\footnotetext{
${ }^{162}$ Hier taucht ein Degenerationsnarrativ auf, dass die ,Unschuld“ der Mit-Primaten, sozusagen den edenischen Urgeschöpfen vor dem Sündenfall, von der post-paradiesischen moralischen Verkommenkeit des Menschen abgrenzt: „Dieser Affe da aber, der wusste, was es besagte, weil er das Gesicht war, weil er war, was er sah, und nicht getrennt war von der Schöpfung, so wie die Mörder getrennt waren, wie die Kinder getrennt waren, wie jeder von uns getrennt und allein ist" (HT, S. 149). Diese bereits in der Syntax an den Duktus religiöser Schriften erinnernde Passage scheint auch Sapolskys judeo-christlichen Traum wiederaufzunehmen und zudem die Levinassche Ethik des Gesichts (vgl. Abschn. 2.1.2) zu reflektieren.
} 
mit den Weisen vom Berg, machten die Männer mit den Mädchen, was Männer immer mit Mädchen gemacht haben“ (150).

Bärfuss' Roman illustriert in dieser Gorilla-Passage, wie das Gefühl des Erhabenen zwar im Angesicht des Gorillas als Modell empfindungs- und affektbasierter Erkenntnis (auch für Nicht-Primatolog/innen) funktioniert. Er hinterfragt es jedoch zugleich in seiner zeitgenössischen Anwendbarkeit bzw. stellt die daraus gewonnene Erkenntnis unter einen grundsätzlichen Verdacht: Das postmoderne Erhabene ist bei Bärfuss nicht mehr als eine spirituelle Egozentrik, die in Analogien verharrt und die der Erzähler zum Zeitpunkt seines Berichts bereits als psychischen Mechanismus durchschaut. Die Realität der reflektierten, instrumentalisierten genozidalen und sexuellen Gewalt lässt die Erfahrung des Erhabenen am Gorilla gerade in ihrer spirituellen Erkenntnismöglichkeit zur narzisstischen Schuld werden. ${ }^{163}$ Sapolskys aus der affektbasierten Erkenntnis entstandene Schwermut angesichts der Verschuldigung des Menschen an den Gorillas und ihrem Habitat wird in der literarischen Reflexion zum Schuldgefühl angesichts der gefühlsgetriebenen Aufwertung des Gorillas und dem Mangel an Empathie gegenüber den Mitmenschen.

\subsection{Affenliebe: Der epistemische Nutzen affektiver Nahformen der Anerkennung}

„Liebe ist die intensivste Anstrengung, sich in die Nähe des anderen zu bringen und sich in dieser Nähe zu halten“. ${ }^{164}$ So definiert Eckart Goebel in seiner Studie zu Jean-Paul Sartres Liebesanalyse einen starken Affekt. Goebels Formulierung

\footnotetext{
${ }^{163}$ Ähnlich gestaltet auch der österreichische Schriftsteller Christoph Ransmayr - allerdings in einem faktualen Text - die Gorilla-Begegnung in seiner Dankesrede für den Würth-Preis als Anlass für eine postkoloniale Reflexion europäischer Grausamkeit an Mensch und Tier in Afrika. Anders als Bärfuss findet Ransmayr jedoch im Blick und im Laut des Silberrücken zum Schluss eine Art Versöhnung, wenn es heißt: „Und wir auf unseren Knien, nachdem unser Herzschlag sich beruhigt hatte und wir zu dem Silberrücken mehr wie Untertanen als Besucher aufsahen, versuchten, [...] die Sprache unseres Gastgebers zu imitieren und ihm unsere friedlichen Absichten mitzuteilen. [...] Gewiß hörte der Silberrücken unseren unbeholfenen europäischen Akzent, den Akzent jener hellen, wässrigen Wesen, die seinesgleichen gejagt, erschossen und geköpft, die teerschwarzen Hände abgehackt und als eingesalzene Trophäen in fernen Hauptstädten der Kultur exportiert hatten, um sie dort präparieren zu lassen und an die Wände muffiger Landsitze zu nageln. Aber dieser Gorilla [...] [s]ah uns an, so lange und so tief hinab in unsere Seelen - oder was immer Europäer in der Brust tragen -, daß wir mit einem Mal ganz die Seinen waren. [...] Er grunzte sanft. Und das bedeutete, so hatten wir es von den Wildhütern gelernt: Es ist gut. Alles ist gut". Ransmayr, Christoph: Dankesrede zum Würthpreis: Europa hat seine Rechnungen aus der Kolonialzeit nie gezahlt. In: Frankfurter Allgemeine Zeitung vom 25.06.2018, Debatten, https://www.faz.net/aktuell/feuilleton/debatten/wuerth-preis-dankesrede-von-christoph-ransmayr-15657111.html?printPagedArticle=true\#pageIndex_0 (14.04.2019).

${ }^{164}$ Goebel, Eckart: Der engagierte Solitär. Die Gewinnung des Begriffs Einsamkeit aus der Phänomenologie der Liebe im Frühwerk Jean-Paul Sartres. Berlin 2001, S. X.
} 
setzt der Liebe ein Ziel, das aus der primatologischen Verhaltensforschung bekannt scheint - sich in die Nähe des anderen (Tieres) zu bringen und in ihr zu halten, im Vokabular der Ethologie: Habituation.

Epistemologische Beschäftigungen mit ,Liebe' konzeptualisieren sie als vielfältige Zugangsweise zu Selbst- und Fremderfahrung und Erkenntnis, als theoretisches Erkenntnisvermögen und habituelles Erkenntnisprinzip. ${ }^{165}$ Dies beginnt, wie Eva Maria Engelen ausführt, bei Platons Konzeption der Erkenntnissuche nach den ,,Spielregeln“ der Erotik der Knabenliebe“, 166 reicht über Augustinus' Liebe als Art des Wissens und Antrieb zur erschöpfenden Suche nach dem höchsten Gute ${ }^{167}$ und Stendhals sieben Stadien umfassenden Prozess der Kristallisation von Wirklichkeit am „Korrelat der Liebe“168 bis hin zu Ludwig Klages’ erotischer Erkenntnisweise der Wirklichkeit und programmatischen gefühlsbetonten Zugängen zur Welt wie bei Martin Bubers Kontrastierung von Ich-Du-Beziehung und Ich-Es-Haltung sowie Levinas' Theoretisierung der epistemologischen Mitsprache des Anderen. ${ }^{169}$ Engelen sieht ,unser[en] Erkenntnisprozess [...] auf das Gefühl angewiesen, um kein sich selbst zerstörender Prozess zu sein“, ${ }^{170} \mathrm{~d}$. h. nicht in unfruchtbaren Skeptizismus zu verfallen. Liebe lässt sich zudem als ein Medium historischen Wandels betrachten - als Ort für einst metaphysische und religiöse Vorstellungen in Sartres Liebesanalyse ${ }^{171}$ ebenso wie bei Luhmann als „symbolisch generalisiertes Kommunikationsmedium“. ${ }^{172}$

Meist wird Liebe in diesem „Konnex zwischen Liebe und Erkennen“"173 in Stellung gebracht gegen objektivierende Formen der (naturwissenschaftlichen) Erkenntnissuche, wie sie eingangs bereits diskutiert wurden (Abschn. 1.2 der Einleitung). ${ }^{174}$

\footnotetext{
${ }^{165}$ Vgl. Kern, Udo: Liebe als Erkenntnis und Konstruktion von Wirklichkeit. „Erinnerung “ an ein stets aktuales Erkenntnispotentital. Berlin/New York 2001, S. 11.

${ }^{166}$ Engelen, Eva-Maria: Erkenntnis und Liebe. Zur fundierten Rolle des Gefühls bei den Leistungen der Vernunft. Göttingen 2003, S. 32.

${ }^{167}$ Vgl. Engelen: Erkenntnis und Liebe, S. 62.

${ }^{168}$ Engelen: Erkenntnis und Liebe, S. 110.

${ }^{169}$ Vgl. Engelen: Erkenntnis und Liebe, S. 110, 159. Zu Buber siehe auch Abschn. 2.4, Anm. 43.

${ }^{170}$ Engelen: Erkenntnis und Liebe, S. 7.

${ }^{171}$ Siehe Sartre, Jean-Paul: L’Être et le Néant. Essai d'ontologie phénoménologique. Paris 1943; Goebel: Der engagierte Solitär, S. 67.

${ }^{172}$ Luhmann, Niklas: Liebe als Passion. Zur Codierung von Intimität. Frankfurt a. M. 1984, S. 21.

${ }^{173}$ Schilling, Rainer: Liebe als Erkenntnisweise. Aspekte der Liebe im Verhältnis zur objektivierenden Naturerkenntnis. Darmstadt 2005, S. 47.

${ }^{174}$ Nicht nur die Lebensphilosophie, auch eine wissenschaftskritisch informierte Erkenntnistheorie problematisiert die Ausdifferenzierung von Gefühl und Vernunft in der Moderne. Schilling spricht von der „Einkerkerung der Liebe in die Innerlichkeit des modernen Subjektes“ als Grundlage für den ,Siegeszug einer Erkenntnisweise [...], die die höchste emotionale Zurückhaltung vom Subjekt abverlangt und alle Teilnahme am objektivierten Gegenstand der Erkenntnis in das Reich der Täuschungen verdammt hat". Schilling: Liebe als Erkenntnisweise, S. 13 f. Engelen spricht gar von einer „Verlustgeschichte“, die mit dem Bedeutungsverlust affektiver Einstellungen und Erlebnisse auch einen Verlust von Erkenntnissicherheit verknüpfe. Engelen: Erkenntnis und Liebe, S. 8.
} 
Liebe als , affektive Nahform“ spielt in der Anerkennungstheorie zudem eine fundamentale Rolle als intersubjektives Medium der Konstitution von Subjektivität. ${ }^{175}$ Vor dem Hintergrund einer solchen Tradition der epistemologischen und anerkennungstheoretischen Wertschöpfung aus der Emotion und der Zielsetzung der Liebe als Nähe-Produktion bietet es sich an, auch im Zusammenhang der ,Trimates ' und ihres Umfeldes danach zu fragen, ob bzw. wie Liebe oder genereller gesprochen ,affektiv[e] Nahformen der Anerkennung"176 hier zu einer Arbeitsweise gehören, die durch Nähe und Vertrauen zwischen Beobachtenden und Beobachteten fundiert ist und Emotionalität epistemologisch instrumentell verwendet.

Anders als beim Gefühl des Erhabenen und seiner (post-)modernen Entwicklung lässt sich der ,Liebe' in den Texten der frühen primatologischen Feldforschung nicht anhand eines Modells nachspüren. Primär soll im Folgenden daher an den Texten von Biruté Galdikas, Jane Goodall und Dian Fossey untersucht werden, welche Rolle verschiedene affektive Nahformen der Anerkennung spielen, und wie sie in die Darstellung des Forschungsprozesses elementar integriert werden. Affektive Nahformen, so wird sich zeigen, tauchen in diesen autobiographischen Feldforschungsberichten auf erstens als Untersuchungsgegenstand und Differenzkriterium, zweitens als Forschungsbedingung und drittens als empathische Erkenntnisweisen. Alle drei Funktionen sind Teil jener bereits angerissenen abendländischen Epistemologie, die die „Liebe als Erkenntnisweise“ (Rainer Schilling) vielfältig erkundet hat. Das Besondere an der primatologischen Erkenntnisgewinnung unter Zuhilfenahme affektiver Nahbeziehungen ist nun, dass hier die in der Erkenntnistheorie genutzten Modelle der Liebe - die platonische Liebe, die augustinische Liebe zu Gott, die romantische Liebe Stendhals, oder auch die mütterliche Liebe der Entwicklungspsychologie - auf das Problem „Affe“ treffen. Damit droht den Primatolog/innen, eingebettet in den kulturellen Kontext der Mensch-Tier-Beziehung, der Verdacht der Zoophilie ebenso wie der Vorwurf der Sentimentalität.

Im Fokus der feministischen Wissenschaftskritik stehen besonders Konstruktionsmechanismen, die Wissenschaft als reine Verstandestätigkeit und ,impersonal“ produzieren, während sie ,a deeply personal as well as a social activity" sei. Fox Keller: Reflections, S. 7.

${ }^{175}$ Vgl. Olschanski, Reinhard: Phänomenologie der Mißachtung. Studien zum Intersubjektivitätsdenken Jean-Paul Sartres. Bodenheim 1997, S. 433.

${ }^{176}$ Olschanski: Phänomenologie der Mißachtung, S. 12. Unter affektiven Nahformen verstehe ich jene Primärbeziehungen, wie sie Reinhard Olschanski in der Folge Axel Honneths aufzählt: „,neben den erotischen Liebesbeziehungen auch enge Freundschaften und die engeren Familienbezüge. Der besonderen Nähe entspricht dabei, daß sie nur zu wenigen Menschen unterhalten werden können“ (S. 377). 


\subsubsection{Prüfstein affektiver Wahrnehmung und Kriterium des Menschlichen: Jane Goodall und die romantische Liebe}

In Jane Goodalls ersten Forschungsmemoiren In the Shadow of Man herrscht nicht die Liebe zur Erkenntnis, wie sie in der „epistemischen Erotik Platons“"177 - als Streben und Begehren nach Wahrheit, nach dem Schönen und Guten und nach Unsterblichkeit - wurzelt. Was Jane Goodall immer wieder formuliert, ist eine augustinische Liebe zum Tier, die sie nach mehr Wissen über dieses suchen lässt: „,[M]y love of the chimpanzees“ (75) geht mit „deep appreciation and understanding of animals“ (75) einher. ${ }^{178}$ Ganz dezidiert ist es jedoch in In the Shadow of Man nicht nur ein auf erweiterter Nächstenliebe gründendes affektiv-ethisches Verhältnis zu Tieren, sondern das eigene emotionale Erleben, das ihre Forschung in Teilen leitet. Dabei liefert Goodall sich ihrer Affektivität nicht einfach aus, sondern sieht die affektive Wahrnehmung - die Wahrnehmung qua Affekt und die Wahrnehmung ihrer eigenen Emotionen - als ebenso überprüfbaren Erkenntnisgegenstand an wie andere Beobachtungen.

Dieses emotionale Erleben betrifft zunächst Goodalls intraspezifisches Verhältnis zu einem anderen Menschen, wird jedoch in einem zweiten Schritt theoriebildend auf den Bereich der Schimpansen extrapoliert: Als Goodall sich in Gombe in den Fotografen und Filmemacher Hugo van Lawick verliebt, wird dies nach seiner Abreise angezeigt durch eine Veränderung ihrer affektiven Disposition: „When Hugo left at the end of November I was alone again. I still was not lonely, yet I was not as completely happy in my aloneness as I had been before he came" (75). Hugo ist eine Variable in Goodalls emotionaler Gleichung. Die daraus folgende Wahrnehmung, „Hugo and I were very much in love“ (90), wird jedoch nicht unbefragt akzeptiert: „But was this, we asked ourselves, simply the outcome of being thrown together in the wilds, far from other European society? Would our feelings change, perhaps, when we found ourselves back in civilization again?" (90). Dazu hat die Forscherin Goodall auch eine Hypothese - „We didn't think so“ (90) - und entwirft eine Art Experiment, einen Test für die Wahrhaftigkeit der Liebe und damit für die Verlässlichkeit ihres affektiven Empfindens: „,W]e resolved to test our love. [...] We would meet again in the world of men rather than the world of apes; and then we would know“ (90). „As it turned out", so wird die experimentelle Bestätigung der Hypothese lakonisch zusammengefasst, ,we knew the very moment we were seperated“(90). Aus dem emotionalen Eindruck, „we

\footnotetext{
${ }^{177}$ Engelen: Erkenntnis und Liebe, S. 25.

${ }^{178}$ Goodall führt ihre „love of all things“ später in Reason for Hope auf eine christliche Ethik zurück, deren Wurzeln bis in ihre Kindheit zurückreichen und „the makings of a true naturalist" (S. 6) bewirkt hätten. Sie lässt sich als augustinisch inspirierte Liebe zur Erkenntnis bezeichnen, wie sie Engelen fasst als „Heilung der Aufspaltung in Urteilenden und Urteilsgegenstand, da die Vereinigung von Erkenntnissuchendem und Erkenntnisobjekt, Liebendem und Objekt der Liebe als Ziel [...] verstanden wurde“. Engelen, Erkenntnis und Liebe, S. 16.
} 
were very much in love“, wird durch die Überprüfung dank einer Veränderung der experimentellen Bedingungen eine Erkenntnis.

Diese verändert ihrerseits die Perspektive der Forscherin: Sobald Goodall van Lawick heiratet, wird nicht nur Gombe zum romantischen Ort, sondern auch die Liebe zum Thema ihrer Forschungsarbeit. Als van Lawick - von Louis Leakey sowohl der National Geographic Society als Dokumentarfilmer als auch Goodalls Mutter als idealer Ehemann für Jane empfohlen - in Gombe eintrifft, liegt Goodalls Fokus zunächst auf männlichen Hierarchien und Dominanzverhalten bei den Schimpansen; bald jedoch wendet sie sich adulten affektiven Beziehungen zu. Im Kapitel „Adult Relationships“ (184 ff.) interessiert sie der Vergleich der „,normal heterosexual relationships that may develop between human beings and those that may be observed between chimpanzees“ (187). ${ }^{179}$ Sie führt zu diesem Zweck verschiedene Szenen der sexuellen Begegnungen und Paarungen an, sowohl unter den beobachteten Schimpansen als auch, abstrakter, in Bezug auf menschliches Sexualverhalten. Diversität ist ihr hier wichtig, denn ,the fact that men and women are capable of establishing and maintaining monogamous relationships both physical and spiritual of long duration“ (187) ist ,far from being the only relationship found between men and women“ (187). Für Goodall ist jedoch gerade diese monogame Langzeitbeziehung ein Differenzmerkmal (,the obvious difference“, 187), nämlich ,a bond [...] not known in the chimpanzee“ (187). Mit diesem ,bond" definiert Goodall expliziter noch die ,wahre Liebe“ als emotionale und spirituelle Erfüllung: ,what we think of as true love - an emotion that encompasses both the body and the mind of the beloved, that mellows with time and brings about harmony of living, that removes any need in the man or the woman concerned for another sexual partner“ (187). Als solche ist sie auch unter Menschen „one of the rarest of human heterosexual relationships“ (187). Damit entwirft sie eine Liebeskonzeption, die Liebe als sexuelle und emotionale evolutionäre Reife imaginiert:

However, although such relationships [wie sie sie unter den Schimpansen beobachtet] may be shadowy forerunners of human love affairs, I cannot conceive of chimpanzees developing emotions, one for the other, comparable in any way to the tenderness, the protectiveness, tolerance, and spiritual exhilaration that are the hallmarks of human love in its truest and deepest sense. (194)

\footnotetext{
${ }^{179}$ Goodalls Fokus auf heterosexuelle Beziehungen leitet sich zum einen aus dem Ideal einer heteronormativen Familientrias ab, welches sie an den beobachteten Schimpansen überprüft und widerlegt. Zum anderen ist es verankert in zeitgenössischen (Moral-)Vorstellungen, aber auch Konzepten sexueller Praktiken. So schreibt sie: „Never, however, have we seen anything that could be regarded as homosexuality in chimpanzees. Admittedly a male may mount another in moments of stress or excitement, clasping the other around the waist, and he may even make thrusting movements of the pelvis, but there is no intromission. It is true, also, that a male may try to calm himself or another male by reaching out to touch or pat the other's genitals; while we still have much to learn about this type of behavior, it certainly does not imply homosexuality" (SM, S. 186 f., Hervorheb. MS). Wie sich an der Dichte von Emphase, Concessio und Prokatalepsis in dieser kurzen Passage zeigt, scheint das Rechtfertigungsbedürfnis bezüglich der Konnotation von Liebe mit Heterosexualität drängend. Dies bleibt jedoch die einzige Passage in In the Shadow of Man, in der Homosexualität eine Rolle spielt.
} 
Der angenommene „lack of consideration for each other's feelings“ bei den Schimpansen ist ,the deepest part of the gulf between them and us" (194), der Grund, weshalb ihnen die ,exquisite awareness of each other's body - let alone each other's mind“ (194) verweigert bleibe: „The most the female can expect of her suitor is a brief courtship display, a sexual contact lasting at most half a minute, and, sometimes, a session of social grooming afterward. Not for them the romance, the mystery, the boundless joys of human love" (194, Hervorheb. MS). Goodall konzipiert hier nicht nur ein Ideal der romantischen Liebe mit affektiven Eigenschaften wie ,tenderness“, ,protectiveness“, ,tolerance“ und ,spiritual exhilaration“ ebenso wie mit prosozialen Zügen (,consideration for each other's feelings“), einer epistemischen Dimension als ,exquisite awareness of each other's body and mind" und Unterhaltungswert (,romance, mystery und boundless joy“). Goodall entwirft auch die Kategorie der erotischen Liebe als Differenzkriterium. Dabei ist ihr jedoch nicht von vornherein daran gelegen, den Menschen als vorzügliche Schöpfung darzustellen. ${ }^{180}$ Sie sucht nach Ähnlichkeiten zwischen Menschen und Affen, z. B. überprüft sie, ob männliche und weibliche Tiere Nester teilen, welche Familienstruktur identifiziert werden kann - und wie sich Kommunikation vollzieht. Gerade in diesem Punkt findet sie Ähnlichkeiten, die ihre Beschäftigung mit der Liebe über die ersten beiden Kategorien (von romantischem Liebesideal und Differenzkriterium) hinausgehen lässt: Die schimpansischen Rufe seien nicht vergleichbar mit der menschlichen Sprache, deren Worte abstrakte Ideen kommunizieren können, aber:

All the same, when humans come to an exchange of emotional feelings, most people fall back on the old chimpanzee-type of gestural communication - the cheering pat, the embrace of exuberance, the clasp of hands. And when on these occasions we also use words, we often use them in rather the same way as a chimpanzee utters his calls - simply to convey the emotion we feel at that moment. ,I love you. I love you.', repeats the lover again and again as he strives to convey his overwhelming passion to his beloved - not by words but by his embraces and caresses. (249)

Sie kommt zu dem Schluss: „This usage of words on the emotional level is as different from oratory, from literature, from intelligent conversation, as are the grunts and hoots of chimpanzees“ (249). Die Reduktion sprachlicher EmotionsäuBerungen auf ihren gestischen Gehalt wird hier um die gestische Kommunikation als adäquate(re)s Medium der Emotionsvermittlung zu einem Bild evolutionärer Gemeinschaft ergänzt.

\footnotetext{
${ }^{180}$ Auch wenn Goodalls Konzeption manches Mal an Schelers Auszeichnung des Menschen gemahnt, der ,ehe er ein ens cogitans [...] oder ein ens volens, ein ens amans" ist. Scheler, Max: Ordo Amoris. In: Ders.: Zur Ethik und Erkenntnislehre. Bern 1957 (Schriften aus dem Nachlass, Bd. 1), S. 345-376, hier S. 356, Hervorheb. im Original. Mit Scheler teilt sie ein spirituelles Verständnis der „primacy of love over knowledge“ in einem Verhältnis zur Welt, in der die Wertschätzung der Wissenserschließung vorausgeht. Vandenberghe, Frédéric: Sociology of the Heart. Max Scheler's Epistemology of Love. In: Theory, Culture \& Society 25/3 (2008), S. 17-51.
} 
Goodalls Konzeption der Liebe dient, so zeigt sich, einem Erforschen der Verwandtschaftsverhältnisse zwischen Mensch und Schimpanse. Werden - gerade durch Goodalls Studien - immer mehr Fähigkeiten und Charakteristika der Schimpansen bekannt, die Menschen zugeschrieben werden, so stehen sie doch im Bereich der hohen Töne der Emotionalität diesen nach. Goodalls eigene Erfahrung einer affektiven Nahform, der Liebe zu ihrem ersten Ehemann Hugo, lässt sie ihr Augenmerk auf bestimmte Formen des Zusammenlebens und der affektiven Interaktion lenken, denen sie bei den Schimpansen nachgeht. Obwohl sie so die, wahre Liebe' als Differenzkriterium behauptet, zeigt sie aber doch auf, dass deren Äußerungen durchaus eng mit denjenigen der Tiere verwandt sind und auch, darin liegt hier die Ironie, die begehrtesten Worte (,I love you. I love you. ') wie gestische Zeichen von evolutionär verankerter, primatischer Emotionalität gedeutet werden können.

\title{
2.4.2 Anerkennung als Bedingung für die Feldforschung: Dian Fossey und affektive Nahformen
}

„Erkennen setzt apriori [sic] Vertrauen voraus“, so benennt Udo Kern das Erkenntnispotenzial von Liebe:

\begin{abstract}
Vertrauen ist epistemische Grundgegebenheit, die in Geltung sein muß, wenn es denn zum ,Erfassen “ von Wirklichkeit kommt. Liebe als fundamentaler Grundakt von Vertrauen konstituiert Vertrauen als apriori [sic] epistemische Grundgegebenheit. Die Liebe geht als fundamentaler Grundakt von Vertrauen auf die Wirklichkeiten den Erkenntnisakten voraus. ${ }^{181}$
\end{abstract}

Dian Fossey hat diese für die Epistemologie prioritäre Rolle des Vertrauens in ihrem Forschungsbericht prominent inszeniert. Die bereits betrachtete Szene der Anerkennung durch das tierliche Andere in der Figur von Peanuts (Abschn. 2.1) eröffnet durch eine Berührung die Möglichkeit der Art von Nahforschung, die Fosseys Gorillastudien begründet. Ganz wesentlich dafür ist, wie bereits dargestellt, die Toleranz des Untersuchungsgegenstands - der Gorillas für die Beobachtungsinstanz; das Mittel, dies zu erreichen, ist Vertrauen durch Gewöhnung (,habituation').

Fosseys affektives Verhältnis zu den Berggorillas hat in der Rezeption ihres Forschungsberichtes, der Vorfälle rund um ihren Tod in Ruanda und in ihrer Mythologisierung durch die National Geographic Society eine Hauptrolle gespielt.

\footnotetext{
${ }^{181}$ Kern: Liebe als Erkenntnis, S. 15. Böhme/Böhme stellen ähnliche Überlegungen als platonisches Erkenntnismodell dar, in dem Erkenntnis die Vertrautheit mit dem Gegenstand zur Veraussetzung hat. In Anlehnung an das Luther-Wort vom Vollzug der leiblichen Liebe als Erkenntnis sprechen sie von „Erkenntnis durch Sympathie“. Böhme/Böhme: Das Andere der Vernunft, S. 279.
} 
Die emotionale Nähe zum Gorillamännchen Digit und Fosseys Umgang mit dessen Ermordung durch Wilderer wurden größtenteils - begünstigt durch die Assoziation mit dem King-Kong-Mythos vom großen dunklen Affen und der weißen Frau - als Äußerungsformen eines Mangels an Objektivität und Wissenschaftlichkeit abgewertet. ${ }^{182}$ Dabei steckt gerade in Fosseys Berichterstattung über Digit deutlich eine Thematisierung ihrer Methoden, deren Nutzen für die Forschung, die sie und ihre Mitarbeiter/innen betreiben, und eine Reflexion ihrer eigenen affektiven Investition in das Forschungsvorhaben. Digit, Poster-Gorilla der Primatologie, ist weniger ein tierliches Liebesobjekt als ein Symbol von Fosseys affektivem Forschungsengagement. ${ }^{183}$

Es mag zunächst problematisch erscheinen, hinsichtlich des affektiven Verhältnisses von menschlichen Beobachtenden und nicht-menschlichen Beobachteten anerkennungstheoretisch zu argumentieren. Legt doch Axel Honneth mit seinem Kampf um Anerkennung eine ,normativ gehaltvolle Gesellschaftstheorie“184 und ein ,,intersubjetivitätstheoretisches Personenkonzept“" vor, ,innerhalb dessen sich die Möglichkeit einer ungestörten Selbstbeziehung als abhängig von drei Formen der Anerkennung (Liebe, Recht, Wertschätzung) erweist". ${ }^{185}$ Der Personenstatus von/für Menschenaffen ist derzeit umstrittener Teil einer Revision des Status der Menschenrechte und der Verhandlung der Kriterien des Subjektes zu Beginn des 21. Jahrhunderts. ${ }^{186}$ Die hier untersuchten Forschungsmemoiren und ihre Verfasser/innen haben Teil an jenem rechts- und sozialphilosophischen Diskurs, denn sie liefern nicht einfach nur epistemische Fakten über die betreffenden Tierarten, sondern folgen darin Koschorkes Erzähltheorie: Ihr Erzählen ist

\footnotetext{
${ }^{182} \mathrm{Vgl}$. bspw. Sapolskys bereits zitierte Einschätzung, Fossey sei zu wenig ,rational' gewesen, $P M$, S. $222 \mathrm{ff}$. Wenig hilfreich, darauf wurde bereits hingewiesen, ist auch die Publikation von Fosseys privaten Aufzeichnungen durch Farley Mowat nicht als systematische und datierte Sammlung, sondern als de- und neukontextualisierte Illustration eines biographischen Narrativs. Siehe Mowat: Woman in the Mists; Whitlock: Remediating Gorilla Girl, S. 484.

${ }^{183}$ Digit sticht unter den Gorillas nicht nur durch die Figurenzeichnung Fosseys hervor, sondern auch, weil er zweifach Berühmtheit erreichte: einmal als ,liebenswertes' Gorillajunges auf Werbeplakaten des ruandischen Tourismusministeriums (,Visit me in Ruanda!“); das andere Mal 1977, als verstümmelte Leiche, als eindrückliches und weitflächig publiziertes Beispiel für das Ausmaß der Wilderei in den Virungas (,Why Ruanda?“).

${ }^{184}$ Honneth, Axel: Kampf um Anerkennung. Zur moralischen Grammatik sozialer Konflikte. Frankfurt a. M. 1992, S. 7, Hervorheb. MS.

${ }^{185}$ Honneth: Kampf um Anerkennung, S. 8, Hervorheb. MS.

${ }^{186}$ Zuvorderst betrieben wird das Anliegen der ,Great Ape Personhood“ von den Philosoph/innen Peter Singer und Paola Cavalieri, dem Rechtswissenschaftler Steven Wise sowie unter aktiver Teilhabe von Primatolog/innen. Vgl. auch Keim, Brandon: Chimpanzees Take a Huge Step Towards (Some) Human Rights. In: Wired vom 21.04.2015, http://www.wired.com/2015/04/ chimpanzee-rights-may-become-reality (12.04.2018) und Feltman, Rachel: Orangutan granted rights of personhood in Argentina. In: The Washington Post vom 22.12.2014, http://www. washingtonpost.com/news/speaking-of-science/wp/2014/12/22/orangutan-granted-rights-of-personhood-in-argentina (12.04.2018); sowie Abschn. 4.5.
} 
nicht bloß eine reproduktive, den erzählten Inhalten gegenüber nachrangige Tätigkeit [...], kein bloßes Rekapitulieren after the fact. [...] Das Bezeichnen interveniert in die Welt, die es scheinbar nur widerspiegelt, und lässt sie in einem kreativen Aneignungsprozess in gewisser Weise überhaupt erst entstehen. ${ }^{187}$

Im Genre der primatologischen Forschungsmemoiren gesellt sich dieser epistemologischen Rückkoppelung auch jene bereits in der Einleitung angeführte performative bei, da hier die Sachverhalte - das Wissen über die behavioralen und kognitiven Charakteristika der Affen - „durch das praktische Handeln der Menschen geschaffen und entsprechend von deren Wahrnehmungs- beziehungsweise Repräsentationsformen abhängig sind" ${ }^{188}$ In den untersuchten Forschungsmemoiren werden die Affen textuell als Subjekte produziert: ${ }^{189}$ Gorillas in the Mist als Erzählung verleiht den Gorillas, die Fossey studiert hat, Personalpronomina (,she“ oder „he“ und nicht „it“) ebenso wie Eigennamen, die nicht randomisiert eingesetzt werden, sondern auf Charakteristika beruhen und in ein Bezugssystem zur Erzählinstanz Fossey (oder ihrem Team) eingebettet sind. ,Whinny“ neigt zum „Whinnying in alarm“ (167); ,Digit“ erhält seinen Namen „because of a twisted middle finger“ (167); ,Uncle Bert" verdankt den seinen einer „remarkable resemblance to a relative of mine“ (168). Bei einem erwachsenen Weibchen heißt es: „Because of her facial expression, I could not help thinking of her as an old goat. Soon Old Goat became her name“ (168). Und ,[t]he third silverback was eventually named Amok for his unstable temperament and his puzzling frequent screaming and running displays" (168). Fossey stellt schließlich als Ergebnis dieser Praxis fest:

The gorillas' names seemed to fit their personalities. Papoose was an adorable snuggly youngster [...]. Mrs. X was always difficult to identify because of her shyness during the first several months. One of the young females had unusual wide, haunting eyes; her name Macho is a Swahili word meaning, eyes'. (169)

Die Identifikation differenzierender und eponymer Charakteristika in den Tieren hat bei Fossey, so zeigt sich, sogar Vorrang vor der ethologischen Methode, matrilineare Angehörige mit dem gleichen Anfangsbuchstaben zu benennen. Die Gorillas werden zudem beschrieben als handelnde, kommunizierende, zumindest mit

\footnotetext{
${ }^{187}$ Koschorke: Wahrheit und Erfindung, S. 22, Hervorheb. im Original.

${ }^{188}$ Koschorke: Wahrheit und Erfindung, S. 23.

${ }^{189}$ Whitlock hat bereits - wenn auch nur kurz - auf die textuelle Produktion der Gorillas als „,biographical subjects" hingewiesen, welche durch ihre Individualisierung, Vergeschlechtlichung und Benennung erfolgt. Whitlock: Remediating Gorilla Girl, S. 478. Siehe auch Shah, Mira: Animal Life Stories; Or, The Making of Animal Subjects in Primatological Narratives of Fieldwork. In: André Krebbe/Mieke Roscher (Hg.): Animal Biographies: Re-making Animal Lives. Basingstoke 2018, S. 119-137.
} 
der vorsichtigen Anmutung von Selbst-Bewusstheit belegte Wesen: ${ }^{190}$ In Fosseys Gorillas in the Mist wechselt homodiegetisches mit im Passiv gehaltenen Passagen eines heterodiegetischen Erzählens; die Fokalisation schwankt dabei jedoch auch in Bezug auf die Tiere zwischen dem behavioristischen Narrativ des ,,vision from without“" und Zuschreibungen, die eine ,,vision from within“-Fokalisation erlauben. ${ }^{191}$ Die Gorillas erscheinen damit als eigenständig handelnde, selbstbewusste Figuren mit Innenleben. Als Figuren einer Erzählung von der Forschungsarbeit sind die Gorillas bei Fossey (und die anderen Affen bei den anderen Primatolog/ innen) daher funktional Subjekte. Als solche können sie Gegenstand anerkennungstheoretischer Überlegungen werden.

\subsubsection{Habituation als Verhältnis der Anerkennung}

Axel Honneth untersucht in seiner Hegel-Rekonstruktion in Kampf um Anerkennung drei Anerkennungsformen, die eine ungestörte Selbstbeziehung des Subjektes generieren und damit die Vergesellschaftung durch praktische Intersubjektivität sichern. ${ }^{192}$ Dabei unterscheidet Honneth im Rekurs auf George Mead, Max Scheler und Helmuth Plessner diese Formen sozialer Integration danach, „ob sie [die soziale Integration] auf dem Weg emotionaler Bindungen, der Zuerkennung von Rechten oder der gemeinsamen Orientierung an Werten zustandekommt“. ${ }^{193}$ Diese drei Formen sind: Liebe, Recht und Solidarität. Den Begriff ,Liebe“ leitet Honneth zwar zunächst aus Hegels Betrachtung des Geschlechterverhältnisses ab, in dem Sexualität als erste Form der Vereinigung einander entgegengesetzter Subjekte gilt. ${ }^{194}$ Liebe, als eine Beziehung, in der das Vertrauen geschaffen wurde, dass dieses Verhältnis wechselseitig ist, ist darauf aufbauend ,ein Verhältnis der wechselseitigen Anerkennung, in dem zunächst die natürliche Individualität der Subjekte Bestätigung findet“. ${ }^{195}$ Honneth empfiehlt darüber hinausgehend jedoch eine möglichst neutrale Verwendungsweise des Begriffs und subsumiert ,alle Primärbeziehungen [...], soweit sie nach dem Muster von erotischen Zweierbeziehungen, Freundschaften und Eltern-Kind-Beziehungen aus starken Gefühlsbindungen zwischen Personen bestehen“. 196

Wie passt nun in diese Auflistung die Forschungsbeziehung zwischen einer Ethologin und einem Gorilla? Zunächst lässt sich diese Beziehung als Vertrauensverhältnis

\footnotetext{
${ }^{190}$ Als Fossey Digit einen Spiegel gibt, zeigt Digit ,acceptance and apparent pleasure when gazing intently at his reflection“ (GM, S. 182). Fossey rät jedoch zur Vorsicht vor bewusstseinstheoretischen Kurzschlüssen: ,It would be presumptive for me to believe he recognized himself. Perhaps the lack of scent clues informed him of the absence of another gorilla“.

${ }^{191}$ Vgl. Hühn, Peter/Pier, John/Schmid, Wolf/Schönert, Jörg (Hg.): Handbook of Narratology. Berlin/New York 2009, S. 115 f.

${ }^{192} \mathrm{Vgl}$. Honneth: Kampf um Anerkennung, S. 7 ff.

${ }^{193}$ Honneth: Kampf um Anerkennung, S. 152.

${ }^{194}$ Vgl. Honneth: Kampf um Anerkennung, S. 63 f.

${ }^{195}$ Honneth: Kampf um Anerkennung, S. 64.

${ }^{196}$ Honneth: Kampf um Anerkennung, S. 153.
} 
strukturieren, in dem die beteiligten Subjekte sich als Nicht-Feinde anerkennen. Wie an der Namensgebungspraxis deutlich wird, treffen Fossey und ihre Studierenden in Karisoke charakterlich unterscheidbare Gorillasubjekte an, die in Gruppenverbänden organisiert sind. Die beobachteten Gruppen sind zudem unterschiedlich und instabil konstituiert: So findet sich die typische polygyne Struktur (Nunkies Gruppe) ebenso wie eine Großfamilie (Gruppe 4) oder ein zeitweiliger Männerbund (Peanuts Gruppe) (GM, S. xix-xxii). Die Gruppen verhalten sich dementsprechend divers nach innen und nach außen hin. Menschen werden zuallererst als Eindringlinge und Gefährdung wahrgenommen. Als wesentlich für die Etablierung einer Feldsituation, die längere Verhaltensbeobachtungen ermöglicht, indem sie weder die Menschen gefährdet noch die Tiere flüchten lässt, identifiziert Fossey die Unauffälligkeit der Beobachtenden, die Aufmerksamkeit für die Bedürfnisse der Tiere und die daraus resultierende Gewöhnung der Gorillagruppen an ihre Beobachter/innen. Diese Kriterien können durchaus lebenswichtig sein, wie sie schreibt: „At times, students as well as I have unexpectedly encountered gorillas before we were aware of the animals' nearness. Such occasions could provoke charges“"(54). Der Gorilla-,Charge', die aggressive Drohgebärde als Urszene des Gorilla-Mythos (siehe Du Chaillu), resultiert in Fosseys Darstellung immer aus einer Unachtsamkeit der Beobachtenden: „Like all charges, this one was really my fault for having climbed the steep slope to approach directly beneath the animal without first identifying myself. Other charges have occured when students, also accidentally, made the same error" (56, Hervorheb. MS). Der Angriff kann jedoch meist beendet werden, weil die Gorillas mit der Zeit ihre Beobachtenden erkennen können: „Upon recognizing me, the group's dominant silverback swiftly braked to a stop three feet away“ (55, Hervorheb. MS); „People who hold their ground usually are not hurt unless they are unknown to the gorillas" (56, Hervorheb. MS); „when he recognized a familiar observer" (56, Hervorheb. MS). Der Vorgang der Habituation in Gorillas in the Mist ist sowohl eine Gewöhnung der Tiere an ihre Beobachter/innen als auch eine Gewöhnung der Beobachter/innen an die Bedürfnisse und Eigenarten der Tiere. In dem Maße, wie die Forschenden lernen, einzelne Gorilla-Individuen (zunächst an Nasenformen, dann an Persönlichkeiten) zu unterscheiden, lernen die Gorillas offensichtlich, einzelne menschliche Individuen in einem FreundFeind-Schema zu unterscheiden. Das Vertrauensverhältnis beruht dabei auf Reziprozität: Können die Gorillas darauf vertrauen, von den Forschenden nur beobachtet und nicht bedroht zu werden, können diese wiederum darauf vertrauen, dass es bei der Drohgebärde statt des tödlichen Angriffs bleibt. Damit ist der Grundstein gelegt für eine auch affektive Beziehung zwischen Beobachtenden und Beobachteten, ,once the trust of the gorillas has been gained“ (167). Diese bildet die Bedingung sowohl für die Beobachtung von individuellem und Gruppenverhalten an sich, als auch für die daraus zu gewinnende Erkenntnis über Berggorillas als Spezies. ${ }^{197}$

\footnotetext{
${ }^{197}$ Ähnlich verhält es sich auch mit den eher vereinzelt lebenden Orang-Utans und Galdikas, die dies noch deutlicher formuliert: „Clearly TP had become habituated, but so, I realized, had I. The process was reciprocal. Gradually, TP and I worked out an unspoken agreement. If he didn't want me to advance, he would angrily slap or shake the vegetation near him until I stopped moving. I learned that if I didn't make eye contact with TP, I could come within ten feet“. $R E$, S. 184.
} 


\title{
2.4.2.2 Digit
}

Dian Fossey führt Digit und die Gruppe 4 der Gorillas erst im 9. Kapitel ihres Berichtes an. Der „ball of fluff“ (167) ist ,[o]n our first meeting“ (168) ca. fünf Jahre alt. Fossey hat die Chance, mit ihrem Team das Gorilla-Männchen durch die Maturität bis ins Erwachsenenalter hin zu begleiten. Dies gibt ihr die Möglichkeit, noch einmal die Herangehensweise der Verhaltensbeobachtung in Karisoke und ihre narrative Bündelung in ,Life Stories` bzw. ,Group Stories` am Beispiel darzustellen.

Die verdeckte Verhaltensbeobachtung (,from a hidden position“, 172; „obscured contacts“, 172) lässt sich nicht unbegrenzt durchführen, da Menschen Gorillas in ihrer Umweltwahrnehmung bei Weitem unterlegen sind. Doch Fossey schlüsselt deutlich auf, wie sich Beobachtung und beobachtetes Verhalten beeinflussen. So sei das Spiel- ebenso wie das Sexualverhalten ,one of the first activities inhibited by the presence of an observer until the gorillas become fairly well habituated" (172). Gerade bei Digit und seinen Halbschwestern hängt ,,[t]he freedom of their play [...] to a great extent“ vom ,type of contact I initiated with the group“ (172) ab:

\begin{abstract}
During obscured contacts, when Group 4 did not know I was observing them, Digit and his younger sisters engaged in prolonged wrestling and chasing sessions [...]. During open contacts, when the group members knew I was present, a great part of the immatures' play behavior involved response reactions such as chestbeating, foliage whacking, or strutting. Each individual seemed to be trying to outdo the other in attention-getting actions. (172)
\end{abstract}

Hier scheinen die Gorilla-Jungtiere für Fossey eine Performance aufzuführen, die deutlich auf eine Reaktion der Beobachtenden ausgelegt ist. Die Beobachter/innen erlangen mit der Gewöhnung der beobachteten Gorillas eine eigene Rolle:

\footnotetext{
I received the impression that Digit really looked forward to the daily contacts with Karisoke's observers as a source of entertainment. Eventually he showed that he could tell the difference between males and females by playfully charging and whacking men but behaving almost coyly with women. (182)
}

Mit dieser Art von teilnehmender Beobachtung wird ein schmaler Grat beschritten zwischen den Gefahren der Beeinflussung des Gorillaverhaltens durch die sichtbaren Beobachter/innen, die zu Bezugs- und Interaktionspersonen für die Gorillas werden, und des Verlusts einer wissenschaftlichen ,Objektivität', gedacht als emotionale Distanz. Fossey geht darauf direkt ein:

\footnotetext{
He seemed pleased whenever I brought strangers along and would completely ignore me to investigate any newcomers by smelling or lightly touching their clothing and hair. If I was alone, he often invited play by flopping over on to his back, waving stumpy legs in the air, and looking at me smilingly as if to say, ,How can you resist me?'At such times, I fear, my scientific detachment dissolved. (182, Hervorheb. MS)
}

Im Beobachtungsverhältnis wird auch für die Forschende aus dem ,detachment“ durch die Interaktion, der sie sich ausgesetzt sieht, ein ,attachment“. Der Gorilla 
erkennt sie nicht nur; er anerkennt Fossey als (Spiel-)Partnerin. Hier verwirklicht sich jene Form der sozialen Selbsterkenntnis, die Honneth in seiner Lektüre von George Meads Sozialpsychologie der Anerkennung identifiziert: „[D]urch die Fähigkeit, in sich die Bedeutung wachzurufen, die das eigene Handeln für den Anderen hat, wird dem Subjekt zugleich die Möglichkeit eröffnet, sich selber als ein soziales Objekt der Handlungen seiner Interaktionspartner zu betrachten“" ${ }^{198}$ Eine solche Erkenntnis bewirkt jedoch noch mehr als diese Form der Einsicht in die eigene soziale Objekthaftigkeit. Die Realisierung der Bedürfnisse der beobachteten Gorillas gleicht einem normativen Akt: der Übernahme der ,,sozialen Handlungsnormen des ,generalisierten Anderen “", 199 durch die, darauf hat Shirley C. Strum mit ihren Befürchtungen zu ihrer sozialen Rolle für die Paviantruppe deutlich hingewiesen (vgl. Abschn. 2.2.3), das Forscher/innensubjekt ,zur Identität eines sozial akzeptierten Mitglieds seines Gemeinwesens gelangen“200 kann. Die Forschungssituation, in der Fossey und ihr Team die Berggorillas beforschen, ist in diesem Sinne nicht nur ein Vertrauensverhältnis; sie schafft auch einen „,sozialen Kooperationszusammenhang“" ${ }^{201}$ In Karisoke sind Fossey, ihre Studierenden und Mitarbeiter/innen durch den engen Kontakt mit einer anderen Spezies noch einmal in die Situation von Heranwachsenden geworfen, die die Handlungsnormen eines sozialen Kooperationszusammenhanges erst neu lernen und anerkennen müssen. ${ }^{202}$

Bei Honneth verläuft die Erweiterungsrichtung von der intersubjektiven Anerkennung in den unter ,Liebe' subsumierten affektiven Nahformen zur rechtlichen Subjektivität der Anerkennung in der Gemeinschaft (bei Hegel, so Honneth, sind Recht und Liebe der Solidarität ,,vorgeordnet[e] Anerkennungsweisen“203). In der Primatologie ist jedoch die Richtung umgekehrt: vom Kooperationszusammenhang der Forschungssituation (,die intersubjektive Gewährung von Rechten“204) zur individuellen affektiven Nahform als Fokussierung des Forschungsaugenmerks (,die Bestätigung der individuellen Besonderheit jedes einzelnen“205) und Fürsorge für den Forschungsgegenstand.

\footnotetext{
${ }^{198}$ Honneth: Kampf um Anerkennung, S. 119. Vgl. auch Donna Haraways Erläuterungen zur Habituations-Erfahrung der der nächsten Forscherinnen-Generation angehörenden Primatologin Barbara Smuts, Haraway, Donna J.: When Species Meet. Minneapolis/London 2008, S. 23 ff.

${ }^{199}$ Honneth: Kampf um Anerkennung, S. 126.

${ }^{200}$ Honneth: Kampf um Anerkennung.

${ }^{201}$ Honneth: Kampf um Anerkennung, S. 146; vgl. auch Haraway: When Species Meet, S. 24 f.

${ }^{202}$ Hier zeigt sich die Habituation der Ethologie strukturanalog zur teilnehmenden Beobachtung in der ethnologischen Feldforschung. Auch dort, das wurde bereits reflektiert, durchlaufen Forschende methodisch bedingt „eine Art zweiter Sozialisation“ in der fremden Kultur. Kohl: Ethnologie, S. 112.

${ }^{203}$ Honneth: Kampf um Anerkennung, S. 146.

${ }^{204}$ Honneth: Kampf um Anerkennung, S. 142.

${ }^{205}$ Honneth: Kampf um Anerkennung, S. 142.
} 
Diese affektive Nahform realisiert sich z. B. in den ,very mixed feelings“ (183), die Fossey anführt, als es darum geht, dass in einer landesweiten ruandischen Tourismus-Kampagne eine Aufnahme „of my lovable Digit“ (183) auf Poster gedruckt wird: „Heretofore Digit had been ,unknown“, only a young male maturing within his natal group. Suddenly his face was everywhere. I could not help the feeling that our privacy was on the verge of being invaded“ (183). „Our privacy“ ist hier Mantelbegriff für einen Beziehungskomplex, der sowohl die besondere affektive Beobachtungsbeziehung von Fossey und Digit als auch die Privatheit der Gorillagruppe (,I certainly did not want the public flocking to Group 4, especially at a time when the group promised finally to become a secure and integral family unit“, 183) und die Exklusivität der Forschungssituation des Karisoke-Teams umschließt.

Mit der Geschlechtsreife und der ,Life Story“ (eine schwere Kampfwunde, die Rolle als ,blackback‘ in seiner Gruppe etc.) verändert sich Digits Persönlichkeit besonders in ihren affektiven Aspekten: Er wird „listless and apathic“ (186), „morosely“ „brooding“ und zeigt eine „,present mood of deep dejection“ (194). Digit verliert das Interesse an Menschen. Fossey berichtet nun von „,considerable relief, for I had worried about his becoming too people-oriented“ (186): Sie ist sich der den affektiven Nahbeziehungen innewohnenden Problematik bewusst. An einem regnerischen Tag, so schreibt sie, „I resisted the urge to join Digit, who was huddled against the downpour and about thirty feet apart from the other animals. It had been months since he had shown any interest in observers, and I did not want to disrupt his growing independence“"(199). Doch auch hier wird wieder deutlich, dass sich die Beobachtungsbeziehung in Karisoke als intersubjektive Anerkennungsbeziehung gestaltet; denn zwar kann Fossey sich selbst beherrschen, doch sie unterschätzt die Handlungsautonomie des Gorillas: „After a few minutes, I felt an arm around my shoulders. I looked up into Digit's warm, gentle brown eyes. He stood pensively gazing down at me before patting my head and plopping down by my side“"(199). Die Zweideutigkeit von Formulierungen der familiären Intimität, die hier vorliegt, weicht der Zweckmäßigkeit, die Fossey in die Szene legt: „I lay my head on Digit's lap“ heißt es zunächst, dann wie rechtfertigend, ,a position that provided welcome warmth as well as an ideal vantage point from which to observe his four-year-old neck injury“ (199). Sie versucht die Narbe zu fotografieren und nimmt ein Gähnen Digits auf, das im vollen Anblick seines Gebisses aus dieser intimen Szene des reziproken Vertrauens ein widersprüchliches Artefakt entstehen lässt: „,my gentle Digit as a King Kong monster“ (199).

Gerade an dieser Beschreibung lässt sich auch die Erkenntnisstruktur von Liebe ablesen, wie sie Rainer Schillings Lektüre von Martin Bubers Kontrastierung des objektivierenden Zugangs zur Welt (Ich-Es-Haltung) mit einem liebenden Zugang (Ich-Du-Beziehung) erforscht. ${ }^{206}$ Durch die Formen der

\footnotetext{
${ }^{206}$ Vgl. Buber, Martin: Ich und Du. Leipzig: Insel 1923. Buber konstatiert dem Menschen zwei Haltungen im Umgang mit der Welt: Die erste Welt ist die ,Eswelt", in der das Gegenüber als Ding und Gegenstand erfasst, verglichen, eingeordnet, beschrieben und zergliedert wird und der
} 
Anerkennung, die die spezifische Forschungssituation in Karisoke erst ermöglichen, wird aus der im besten Fall phänomenologischen und utilitaristischen Ich-Es-Haltung des Forschungsvorhabens die Ich-Du-Beziehung zu einem ganzen Wesen. Sie ermöglicht eine „Anerkennung des Anderen als Anderen, sie wird ihm als diesem Anderen gerecht oder trachtet zumindest danach, ihm gerecht $\mathrm{zu}$ werden. "207 Erst in der Verallgemeinerung von der Sammlung an Verhaltensbeobachtungen einzelner und in Gorillas in the Mist individuierter Gorillasubjekte als Akteur/innen in der Forschungssituation zu generalisierbaren Charakteristika einer Spezies kehrt die Ich-Es-Haltung der Forscher/innen wieder zurück: in der primatologisch notwendigen und erwünschten Abstraktion von Digit auf die Berggorillas.

\subsubsection{Solidarität}

In seiner Phänomenologie der Missachtung hebt Reinhard Olschanski die Besonderheit der affektiven Nahformen darin hervor, dass die Erfahrung der Anwesenheit des Anderen in ihnen ,sich dabei bis hin zu den affektiven Modi des ,Verschmolzenseins“ oder des ,Einsfühlens““208 erstrecken kann. Diese Art von auflösender Vereinigung, wie sie in der Epistemologie der Liebe zur Voraussetzung für Erkenntnisweisen gemacht wird, lässt sich bei Fossey nicht finden. Sehr wohl drängt sich jedoch am Beispiel Fosseys ein anderer Aspekt auf, den Olschanski aufgezeigt hat, jener der „Welterschließung“ durch den oder die Andere/n, die die Verlusterfahrung umso tiefer gestalten kann: „,S]o kann etwa der Tod [...] eine Lücke in den gesamten Weltbezug des ihm nah Verbunden reißen“; 209 eine ,existenzielle ,Entleerung“, ein sich auf alle Lebensvollzüge ausdehnender Verlust an ,Sinnhaftigkeit“" ${ }^{210}$ ist die Folge. Sicherlich lässt sich dies nicht umfassend strukturanalog auf die Forschungssituation und die Bindung an einzelne beobachtete Tiere beziehen; allein schon, weil über den Weltbezug der Tiersubjekte hier recht wenig gesagt werden kann. ,Welterschließung' ist jedoch für die Forscherin unter den spezifischen Bedingungen der Feldforschungssituation in den Bergen Ruandas durchaus die tagtägliche Erschließung der (Um-)

Erkenntnis dient (S. 38), die zweite ist die ,Duwelt ${ }^{\star}$ und „,[d]er Mensch wird [erst] am Du zum Ich“ (S. 28): „Das Ich des Grundworts Ich-Es erscheint als Eigenwesen und wird sich bewußt als Subjekt (des Erfahrens und Gebrauchens). Das Ich des Grundworts Ich-Du erscheint als Person und wird sich bewußt als Subjektivität“ (S. 60). Bei Buber geht es letztlich um das ,ewige[] Du“ (S. 71) Gottes; aber es findet sich auch eine Derrida inspirierende Passage zum Blick des Tieres (einer Katze): „Die Augen des Tiers haben das Vermögen einer großen Sprache. [...] Eben noch hatte die Eswelt das Tier und mich umgeben, ausgestrahlt war einen Blick lang die Duwelt aus dem Grunde, nun war sie schon in jene zurückgeloschen“"(S. 93). Vgl. Derrida: L'Animal.

${ }^{207}$ Schilling: Liebe als Erkenntnisweise, S. 127.

${ }^{208}$ Olschanski: Phänomenologie der Mißachtung, S. 377.

${ }^{209}$ Olschanski: Phänomenologie der Mißachtung, S. 377.

${ }^{210}$ Olschanski: Phänomenologie der Mißachtung, S. 377. 
Welt der Berggorillas. Fosseys Welt besteht in dieser Situation (und in diesem Text) ${ }^{211}$ nur aus gorillarelevanten Bezügen, selbst wenn sie über das ,Camp Life“ oder ruandische Politik schreibt. Die affektive Beziehung zu einzelnen Individuen wie Macho, Peanuts oder Digit ist also Bedingung für forschungsrelevante Wissensgenerierung durch emotional fundierte, Welterschließung'.

Deutlich wird dies ex negativo durch die Verlusterfahrung. Digits Tod trifft Fossey auf eine Art und Weise, die ihr den Weltbezug raubt:

There are times when one cannot accept facts for fear of shattering one's being. As I listened to Ian's news [dass Digit von Wilderern getötet und verstümmelt worden ist] all of Digit's life, since my first meeting with him as a playful little ball of fluff ten years earlier, passed through my mind. From that moment on, I came to live within an insulated part of myself. (206)

Der anschließende Nachruf auf Digit offenbart die Anerkennung, die Fossey diesem für ihre Forschung an Gorilla-Lebensgeschichten zentralen Individuum zollt: „Digit, long vital to his group as a sentry, was killed in this service by poachers on December 31, 1977“ (206), beginnt diese Passage. Sein Kampf wird im Modus des Heroischen geschildert (,That day Digit took five mortal spear wounds into his body, held off six poachers and their dogs in order to allow his family members, including his mate Simba and their unborn infant, to flee to the safety of Visoke's slopes.“, 206) und ist reich an Vokabeln der Auszeichnung („Digit's last battle had been a lonely and courageous one. During his valiant struggle [...]", 206, Hervorheb. MS). Fossey stellt unmittelbar einen Bezug zu ihrem eigenen Empfinden her: „I have tried not to allow myself to think of Digit's anguish, pain, and the total comprehension he must have suffered in knowing what humans were doing to him“ (206). Vom Nachruf wechselt die Passage in die politische Diskussion zur Frage, ob dieser Tod öffentlich gemacht werden soll, und schließlich in die Kriminalgeschichte, denn Digits Tod wird als Verbrechen dargestellt („Digit's slaying“, 209; „Digit's killing, 211; „Digit's murder“, 212). Schließlich tauchen auch die Ansprüche und Verpflichtungen auf, die Olschanski aus der Nähe der affektiven Beziehung erwachsen sieht, denn:

[F]or countless weeks unable to accept the finality of Digit's death, I found myself looking toward the periphery of the group for the courageous young silverback. The gorillas allowed me to share their proximity as before. This was a privilege that I felt I no longer deserved. (209, Hervorheb. MS)

Fosseys Schutzauftrag und ein Verantwortungsgefühl, das sie zur Streiterin für die Berggorillas werden lässt, erwachsen erst aus der affektiven Bindung an die Tiere. Dieses Verantwortungsgefühl überdauert den Tod Digits und führt zu einer

\footnotetext{
${ }^{211}$ In den Textpassagen aus Fosseys privaten Aufzeichnungen, die Mowat verwendet, scheint der Hauptfokus Fosseys dagegen auf zwischenmenschlichen Interaktionen und der eigenen Befindlichkeit zu liegen; Gorillas sind eher Randfiguren im Drama der Forschungsstation. Vgl. Mowat: Woman in the Mists.
} 
aktivistischen Strategie, die in den Augen vieler „The Madness of Dian Fossey“ (so Montgomerys Kapiteltitel) ${ }^{212}$ darstellt, in ihrer Radikalität im Umgang mit der Abwägung von menschlichen vs. tierlichen Ansprüchen und Bedürfnissen jedoch ein Umdenken der Naturschutzpolitik in Ostafrika provozierte. Sie lässt sich auf Basis von Honneths Hegel-Lektüre auch verstehen als kostspielige (und einseitige) Form der Sittlichkeit, nämlich als „die Art von sozialer Beziehung, die entsteht, wenn sich die Liebe unter dem kognitiven Eindruck des Rechts zu einer universellen Solidarität unter den Mitgliedern des Gemeinwesens geläutert hat":213 Es ist die anspruchsvollste Form der Anerkennung. Bei Fossey wäre dies eine Solidarität bis in den gewaltsamen Tod hinein.

\subsubsection{Empathische Erkenntnisweisen: Galdikas' ,Mutterrolle' für den Erkenntnisgegenstand}

Zeigt Jane Goodalls Beschäftigung mit romantischer Liebe als anthropologischem Differenzkriterium wie aus einer privaten emotionalen Situation ein Forschungsinteresse und -fokus entstehen kann, so legt Dian Fosseys Gorillas in the Mist die Anerkennungsstrukturen frei, die einer ertragreichen Forschungsbeziehung zwischen Menschen und anderen Primaten als zwei hochsozialen Arten nicht allein ethisch, sondern zuvorderst praktisch zugrunde liegen muss. Dabei wird an Fosseys Feldarbeit über ihren Bericht hinaus deutlich, wie affektive Nahformen mit dem Recht und der Solidarität als weiteren Formen von Anerkennung im Austausch stehen - und welche Konsequenzen dies in der Praxis mit sich bringt: Artenschutz geht, so zeigt in radikaler Form Fosseys Forscherinnenbiographie, der Verhaltensbeobachtung voran.

Biruté Galdikas dagegen wurde lange Zeit aufgrund ihrer publizistisch etablierten Rolle als ,Orang-Utan-Mutter' rezipiert: zunächst als Amazone, dann als ,Glucke' des Artenschutzes. ${ }^{214}$ Die ideologische Aufladung der Mutterfigur ${ }^{215}$ zusammen mit jener Form von Primatenforschung und Artenschutz, die Galdikas praktiziert, macht hier die Reduzierung der Forscherin auf die Rolle einer sentimentalisierenden Ersatzmutter für Orang-Utan-Waisenkinder scheinbar einfach. ${ }^{216}$

\footnotetext{
${ }^{212}$ Montgomery: Walking with the Great Apes, S. 214.

${ }^{213}$ Honneth: Kampf um Anerkennung, S. 146.

${ }^{214}$ So ziert sie gleich mit ihrem ersten Artikel im National Geographic Magazine mit einem Orang-Utan-Baby auf der Hüfte und einem weiteren an der Hand das Cover der betreffenden Ausgabe, siehe Galdikas-Brindamour, Biruté: Orangutans. Zu einer solchen Instrumentalisierung des Primatologinnenkörpers vgl. auch wieder Haraway: Primate Visions, S. 148 f.

${ }^{215} \mathrm{Zu}$ Mutterschaft als „master status“, an dem alles Weibliche Handeln und Sein gemessen werde, vgl. Katz Rothman, Barbara: Recreating Motherhood. New Brunswick 2000, S. 7.

${ }^{216}$ Aus diesem Grund führt sie in Montgomerys ,weiblicher Typologie" auch die Sektion „Nurturers" an, während Goodall eher als geduldige „Scientist“ und Fossey als sich aufopfernde „Sorceress-Warrior“ fungiert. Vgl. Montgomery: Walking with the Great Apes, S. 3 ff., 89 ff., $130 \mathrm{ff}$. u. $214 \mathrm{ff}$.
} 
Doch diese ,Mutterrolle‘ ist nicht selbst gewählt, sie entspricht keinem Bedürfnis nach Ersatzobjekten für einen Kinderwunsch. Es ist Galdikas ein Anliegen, dies herauszustellen: „Rod [ihr Ehemann] and I had no immediate plans to start a family“ (196). Galdikas' Forschungsmemoiren arbeiten sich merklich daran ab, ihr feministisches Selbstverständnis als Frau und Wissenschaftlerin im Einklang mit der Forschungspraxis und persönlichen Präferenzen zu erläutern. So setzt sie sich mit den normativen Einschränkungen im Austausch mit den Forschungsassistenten für sie als ,,a young female and their ,boss ' $[. .$.$] in a predominantly Muslim society“$ (107) ebenso auseinander wie mit einer Reflexion ihrer Beweggründe: „I went to Indonesia for so-called ,female" reasons: I wanted to help." Im Gegensatz zu Rod, so schreibt sie, „I wasn't interested in adventure for adventure's sake“ (330). Die Differenz zwischen dem Selbstbild und der Realität der Forschung wird hervorgehoben: „I sometimes found myself with one infant on each side, a smaller infant wrapped around my neck, another infant sitting on my foot, and a gibbon attached to my other ankle. This was not the portrait of an effective professional“" (361).

Es ist Galdikas ein Anliegen, deutlich werden zu lassen, dass sie nicht nach Borneo gereist ist, um Mutter zu werden und in der Mutter-Kind-Dyade emotional involviert zu sein, sondern um als Forscherin zu arbeiten. Die Mutterrolle hilft Galdikas aber letztlich dabei, ein Verständnis der Lebensweisen und Verhaltensspezifika der Orang-Utans zu gewinnen. Mit dem Wissen von den Methoden und Forschungsproblemen der beiden anderen Primatologinnen und mit auf deren Erfahrungen und Narrativen basierenden Strategien kann Galdikas - als letzte ins Feld geschickte ,Trimate " - das Potenzial von Emotionalität für die Forschung an Primaten im Freiland ausbauen: Die Mutterrolle ist ein aus der Forschungssituation erwachsenes Instrument der behavioralen Einfühlung, und Empathie dabei ein epistemologisches Werkzeug in Galdikas' Arbeitsweise.

\subsubsection{Mutterschaft als wissenschaftliche Rolle}

Angereist war Galdikas in Kalimantan gemeinsam mit ihrem Mann Rod Brindamour, um als eine der ersten Forscher/innen überhaupt freilebende Orang-Utans in Borneo zu studieren. Bald wird sie darauf aufmerksam, dass eine Hauptgefahr für die Tiere darin besteht, dass Orang-Utan-Jungtiere als Haustiere begehrt sind. Sie macht sich daran, das bereits bestehende Verbot mithilfe offizieller Vertreter umzusetzen und gefangene Orang-Utans wieder auszuwildern. Dabei wird sie wider Erwarten zur Ersatzmutter der Jungtiere. Das Orang-Utan-Baby ,Sugito“ ist als erstes Exemplar der auszuwildernden Waisen Initiator dieses Vorgangs. Galdikas beschreibt die Konfiszierung des „squeaking infant“ (129) als Urmoment (,,an extraordinary moment“, 129) des neuen Verhältnisses zwischen der Forscherin und ihrem Beforschten: „He squealed piteously, as, one by one, I unpried all four limbs. Once I held him, however, he clung to me with the same strength he had used to resist me seconds earlier" (128). Zu diesem Zeitpunkt, so ist es Galdikas wichtig zu betonen, „I felt no maternal affection for the smelly but firm-bodied bundle in my arms“" (128, Hervorheb. MS). Stattdessen wird sie von einem anderen affektiven Muster motiviert: „Yet I felt exhilerated. We had saved the infant 
from almost certain premature death in captivity“ (128); sie ist „[t]riumphant“ (128) in ihrem Erfolgsgefühl über die vollbrachte Leistung.

Dabei bleibt es jedoch nicht, denn der kleine Orang-Utan betreibt einen körperlichen Aneignungsprozess, der Galdikas affektiv bestimmen wird:

[A]s I walked, Sugito molded his body to mine. I did not even have to touch him; he clung to me entirely of his own accord. (129)

I walked with Sugito clinging to my body for several hours in the dense undergrowth of the great forest. [...] When we returned to the dugout, I still held Sugito. Back in camp, I wanted to take a quick bath [...]. I asked Rod to take Sugito. As he began pulling the infant off my body, Sugito squealed, urinated, and bit, as usual. But this time, try as he might, Rod could not pry him loose. (130 f.)

Galdikas stellt fest: „Something had changed“ (131). Sie fügt das Verhalten des Orang-Utans in ein ihr und ihren Leser/innen bekanntes Muster: „Sugito had decided that he wanted a mother of his own, and the mother he chose was me" (130 f.). Galdikas akzeptiert die daraus resultierende Rolle als notwendig, transformiert sie in eine bewusste Entscheidung (,I had decided that I would be the best possible orangutan mother I could be.“, 139) und füllt sie zunehmend affektiv aus: „Much of the time, Sugito gave me little choice. But most of the time I was enchanted by my adopted infant. I enjoyed the soft feel of his orangutan hair [...]. I liked the firmness of his muscular body“ (139, Hervorheb. MS). Dies ist jedoch nicht die Eröffnung einer innigen Mutter-Kind-Dyade. Denn bald fügt sich die Situation des menschlichen Paares mit dem Orang-Utan-Kind zum ödipalen Drama, denn „he and Rod had a somewhat contentious relationship" (142). Sugito zeichnet sich in diesem Drama aus durch „squealing possessiveness“, ,,jealousy“ (142) und „a strong personal dislike for Rod“ (143), schlicht: „Sugito's archenemy was Rod“ (141). Besonders nach einer Art sexuellem Übergriff auf Brindamour (bei dem Sugito versucht, seinen Penis in Rods Ohr einzuführen; eine Art vergnüglicher Umkehrung des ödipalen Begehrens) enden dessen Bemühungen, diese Feindschaft nicht zu erwidern. Doch Sugito hat Galdikas bereits für sich gewonnen (,Sugito became my infant“, 142), primär durch eine schlichte Landnahme auf Galdikas Körper:

No matter what I was doing or where I was going, Sugito was my inevitable companion [...]. As I walked, Sugito clung to my side or wrapped himself around my neck [...]. When I stopped, he sat in my lap [...]. (139)

If Rod moved a hand toward me during the night, Sugito would instantly wake up, urinate, squeal, and angrily bite Rod. I couldn't touch Rod because Sugito monitored me as well. (142)

Galdikas insinuiert zwar durch ihre Wortwahl ein Befremden an der Manipulation durch das Affenkind - so ist Sugito unvermeidlich (,inevitable“), klammert (,,clung“) und überwacht sie (,monitored me“) -, sie stellt jedoch selbst die scheinbar konträre, aber evolutionär bedingte Wirkung dieser Taktik als Mechanismus heraus: „Sugito's constant presence on my body strengthened my affection for him“ (142). Die somit herbeigeführte affektive Nahform bzw. Versorgungs- 
beziehung nennt Galdikas sogar schließlich „Liebe“, wenn sie von „,a surge of love for him“ (142) schreibt.

In dieser Thematisierung der affektiven Nähe weist Galdikas jedoch darauf hin, dass sie sich der bestehenden Differenz bewusst ist: „I would remember that he was an orangutan and it was a mistake to judge him as a human. And then I would love him even more“ (142). Diese liebevolle Zuneigung erwächst, so zeigt sich in dieser Passage, nicht nur aus der körperlichen Übernahme durch den Orang-Utan, sondern auch aus Sugitos Symbolkraft für Galdikas:

Sugito and I bonded, each day more deeply. My relationship with him became the most important in my life, rivaled only by my relationship with my husband. Sugito was my child and my commitment: in addition to his own appealing self, he represented all orangutans. He symbolized my need, my responsibility, to help the orangutan species. (142, Hervorheb. MS)

Was dieses semantisch ebenso wie affektiv aufgeladene Verhältnis in der Kategorie Eltern-Kind-Beziehung für Galdikas produziert, ist die Möglichkeit von systematisch nutzbarer Ein- bzw. Nachfühlung. Am eigenen Leib und mit affektivem Sensorium kann sie nicht nur erstens beobachten, welches evolutionär bedingte Verhalten sehr junge Orang-Utans zeigen, sondern auch zweitens erfahren, wie sich dieses auf sie als Objekt einer kindlichen Überlebensstrategie auswirkt. In Rückkoppelung mit den Beobachtungen von freilebenden Orang-Utans im Wald lassen sich weitere Einblicke in Verhaltensformen gewinnen und Erklärungsansätze ableiten. Galdikas schreibt z. B., Sugitos Eifersucht sei ihr erst durch die Beobachtung von Mutter-Kind-Kleingruppen verständlich geworden: „Through my observations of wild orangutans, I understood Sugito's jealousy“ (141), denn: „A wild orangutan female lives alone; her son would have no competitors for her affection and attention“"(141). Die Nähe zu den auszuwildernden Waisen verleiht Galdikas einen Eindruck davon, welche Bedürfnisse und Fähigkeiten Orang-Utans haben.

\subsubsection{Narrative Empathie}

Empathische Transferleistungen speisen Galdikas' Bericht über ihre Jahre in Kalimantan und sind die Grundlage für jene ,Life Stories', welche auch in $R e$ flections im Vordergrund stehen. Galdikas versteht, indem sie erzählt - im Sinne jener narrativen Empathie wie sie Kurt Breithaupt konzeptualisiert hat. Für Breithaupt ist Narration ,die Ausnahmeform, in der Empathie zugelassen wird“. 217 Damit beschreibt er die Problematik der Verhaltensforschung treffend: Zur Sinnerzeugung aus einzelnen Verhaltensbeobachtungen gehört ein Verfahren, in dem Empathie nicht als emotionale Auflösung im Anderen, als Perspektivverlust, verstanden wird, sondern als Kalkulation der Handlungsmöglichkeiten des Anderen. Dies ist ein Verstehen der Anderen durch eine Narrativierung, die deren Hand-

\footnotetext{
${ }^{217}$ Breithaupt, Fritz: Kulturen der Empathie. Frankfurt a. M. 2012, S. 12.
} 
lungsakte sequenziell und sinnerzeugend miteinander verbindet und Intentionen unterlegt. ${ }^{218}$

Interessanterweise setzt Breithaupts Modell der narrativen Empathie eine Dreierszene voraus, in der der „Beobachter [...] den Konflikt oder zumindest eine Meinungsverschiedenheit von zwei anderen“ beobachtet und ,über die möglichen Ursachen, Motivationen, Intentionen und Folgen" 219 spekuliert. Damit skizziert Breithaupt nicht nur ein theatrales Zuschauermodell für das Subjekt der Empathie sondern auch eine paradigmatische Situation der Verhaltensforschung: Die Primatologin beobachtet einen sozialen Austausch zwischen zwei (oder mehr) primatischen Individuen und wird das beobachtete Verhalten interpretieren (müssen), um daraus Wissensbestände generieren zu können. Breithaupt sieht als wesentlich für den Einsatz von Empathie-Effekten die Parteinahme in Dreierszenen an. Dem entspricht in der Ethologie zum einen eine methodische Entscheidung für das ,Focal-Animal Sampling', den Fokus auf ein zu beobachtendes Individuum (und seine Interaktionen). ${ }^{220}$ Zum anderen involviert dies ein weiteres Grundproblem der Verhaltensforschung, die trotz aller angestrebter Objektivität in der Beobachtung und Aufzeichnung doch vom ,homo narrans ${ }^{6221}$ durchgeführt wird. Dazu gehört im Fall der Verhaltensforschung eine Mini-Narration mit handelnden Protagonist/innen, die aus dem sozialen Austausch mithilfe literarischer Mittel eine thematische Szene macht und sie eingliedert in übergreifende, Hypothesen stützende Narrative. Wer erzählt, der/die schafft nicht nur Kontingenzbewältigung ${ }^{222}$,als Bewegung in Richtung zur Kausalität“",223 sondern übt sich auch in empathischen Erzählspielen, die das Andere epistemisch erschließen, bzw. „einen anderen emotional oder kognitiv [...] verstehen“224 sollen. Wie Breithaupt darlegt, ist gerade diese Form der Empathie verbunden nicht etwa mit einer Grenzauflösung des Subjektes in emotional überwältigender Einfühlung, sondern mit einer affektiven Kanalisierung, gar mit einer Abwehr. Er spricht sogar vom „,narrativen Kalküll“225 der Empathie.

Ganz entgegen der Erwartung mütterlicher Liebe zeigt sich an Galdikas' Reflections of Eden, dass sie die Affektivität empathischer Erklärungsversuche strategisch eingrenzt; denn sie betreibt jene von Breithaupt identifizierte ,gezielte

\footnotetext{
${ }^{218}$ Vgl. Breithaupt: Kulturen der Empathie, S. 11 f.

${ }^{219}$ Breithaupt: Kulturen der Empathie, S. 12.

${ }^{220}$ „Once chosen, a focal individual is followed to whatever extent possible during each of his sample periods“. Altmann, Jeanne: Observational Study of Behavior: Sampling Methods. In: Behaviour 49/3/4 (1974), S. 227-267, hier S. 242 ff.

${ }^{221} \mathrm{Vgl}$. Koschorkes Hinweis auf die Begriffsprägung in Folge von Walter Fishers kommunikationswissenschaftlichen Publikationen, Koschorke: Wahrheit und Erfindung, S. 9.

${ }^{222} \mathrm{Vgl}$. Koschorke: Wahrheit und Erfindung, S. 11.

${ }^{223}$ Breithaupt: Kulturen der Empathie, S. 136, Hervorheb. im Original.

${ }^{224}$ Breithaupt: Kulturen der Empathie, S. 20.

${ }^{225}$ Breithaupt: Kulturen der Empathie, S. 11.
} 
Begrenzung von Ähnlichkeit“.226 Dies erscheint zunächst widersinnig in einer Primatologie, deren Erkenntnisinteresse zu diesem Zeitpunkt vor allem die Genese menschlicher Frühformen betrifft. Galdikas bedient durchaus dieses Interesse, wenn sie die evolutionäre Nähe der beiden Primatenarten homo sapiens und pongo betont. Orang-Utans sind bei Galdikas ,our closest living relatives“ (397), ,primates, who are so much like ourselves“ (391). Ihr Text arbeitet jedoch zum einen mit Differenzierungen, welche die Tiere in ihr eigenes Recht setzen sollen. So ist der Grund für den Beginn der Orang-Utan-Studien vor allem deren Eigenart: „Sociability is one of the trademarks of the primate order; Why were orangutans different?" (32). Die obig besprochene Passage über Sugito beginnt zudem mit einer Beschreibung, die wenig in das affektauslösende menschliche Kindchen-Schema passt: „his funny Popeye face and spiky hair“ (128) und die „firmness of his muscular body“ (139) unterscheiden ihn nicht nur deutlich vom menschlichen Baby, sie machen ihn zu einer Abweichung. Er ist ,a cute but malformed embryolike creature, an elf of a baby“ (142). Galdikas betont so gerade in jenen Passagen, in denen Sugito vorgestellt wird, die Differenz: „Human babies do not demand this constant contact“ (139. Hervorheb. MS); ,Sugito was different“ (139); „I was human and he was an orangutan“ (139). Jene Ähnlichkeit zwischen Orang-Utan und Mensch, die sich aus evolutionären Zusammenhängen und kognitiven Vorgängen speist, ist nach Galdikas ironischerweise ,what makes them attractive as pets. We see them as ,cute", because they are almost human" (136, Hervorheb. MS). Dies jedoch, die Liebe zum uns möglichst ähnlichen Haustier, wird von ihr direkt in die Ökonomie der Wilderei eingebunden: „,This, in turn, makes orangutans more valuable to poachers. The people who want a pet orangutan to ,love " are directly responsible for their slaughter" (136).

\footnotetext{
${ }^{226}$ Breithaupt: Kulturen der Empathie, S. 21. Breithaupt geht explizit darauf ein, dass Empathie zwar die Ähnlichkeitskonstruktion benötigt, um entstehen zu können und zum Verstehen zu führen. Er differenziert dazu sogar etliche Ebenen, auf denen Ähnlichkeit zwischen Beobachtenden und Beobachtetem ,unterstellt‘ werden kann. Erstens kann Ähnlichkeit jedoch nie Gleichheit sein, ein bereits von Thomas Nagels bewusstseinstheoretischem Fledermaus-Aufsatz thematisiertes Problem sowohl der Anthropomorphisierung als auch der Epistemologie, vgl. Nagel, Thomas: What It Is Like to Be a Bat? In: The Philosophical Review 83/4 (1974), S. 435-450. Zweitens drohen durch „Fehlbefunde der Ähnlichkeit“ sowohl emotionale Ansteckung, die die Differenz „,zwischen mir und dem anderen in Bezug auf die Emotionen“ auslöscht, als auch vom Ich auf alle Anderen verallgemeinernde Projektionen, die ein ,akkurates" Verstehen des Anderen verstellen, vgl. Breithaupt: Kulturen der Empathie, S. $25 \mathrm{ff}$. Schutz vor beidem bietet, so schlägt Breithaupt vor, die Reflexion des „Ichs“ als „Funktion des Unterbrechens der [emotionalen] empathischen Gleichheit“ dort, wo das vorhergesagte Handeln des Beobachteten von der Erwartung abweicht (S. 53). In diesem Sinne spricht er auch von einer ,intellektuellen ' Empathie, die in diesem Prozess die emotionale ablöse. Das bis auf Adam Smith und Gotthold Ephraim Lessing zurückgeführte Vorhaben, in der epistemologischen Betrachtung von Empathie nicht von „Strukturen der Identität“ auszugehen, sondern danach zu fragen, ,wie diese Differenzierung selbst Effekt von Empathie ist“, und somit die „Empathie als Blockade“ (S. 54) zu untersuchen, scheint mir für eine Untersuchung der Emotionalität in der frühen primatologischen Arbeitsweise vorbildhaft.
} 
Zum anderen ist es Galdikas wichtig, die Grenzen empathischer Leistung aufzuzeigen. Obwohl sie sich als den Orang-Utan-Weibchen ähnlich (und daher zur Forschung geeignet) betrachtet (,In equating peace and tranquility with solitude, I had more in common with Beth and with orangutans than with these [Dayak] women or most other traditional peoples", 103), bleibt deren Bewusstsein ihr verschlossen: „The emotional aloofness of the orangutan females was alien to me. I couldn't have penetrated their universe even if I could have climbed up into the canopy with them. We were divided by at least ten million years of separate evolution" (250, Hervorheb. MS). Selbst beim privilegierten Pflegekind und Symbol-Orang-Utan Sugito springt die Erwartungsverletzung ein, um eine emotionale Vereinnahmung durch eine kritische subjektive Perspektive zumindest zu stören: „He seemed to need me so much, it was almost unbearable, he clung to me night and day. While endearing, it was also claustrophobic, especially when I was trying to transcribe notes, clean the hut, or do other work" (139). Gerade die Raumnahme des Affenkindes auf ihrem Körper wird von Galdikas' ,Ich ‘ - dem mütterlichen Gefühlsauftrag zuwiderlaufend - als Verletzung ihrer Integrität als arbeitender Forscherin wahrgenommen. Das Forscherinnen-Ich wehrt sich gegen die emotionale Vereinnahmung und ermöglicht so einen reflektierteren Blick auf den Forschungsgegenstand.

\subsubsection{Empathie und Forschungsethik}

Das letzte Kapitel von Galdikas' Reflections of Eden widmet sich unter dem Titel „Kin“ (379 ff.) abschließend vor dem Hintergrund einer Reflexion der Forschungsgeschichte der ,Trimates' der Problematik einer richtigen Form von Empathie in der Feldforschung. Darin geht Galdikas ausführlich auf Dian Fossey und deren Tod ein und diskutiert an Fosseys Gorilla-Forschung die Konstitution eines ethologischen Begreifens des Tieres, die an Breithaupts Differenzierung von ,subjektiver' und ,akkurater' Empathie erinnert:

I've been quoted as saying that Dian ,became a gorilla'. I meant this metaphorically. Dian never thought that she was a gorilla. But to some degree she did learn to think like a gorilla, and she sometimes behaved like a gorilla. Her empathy with her subjects went beyond expertise. In time, she became accepted almost as a family member in gorilla groups. Even her harshest critics admitted that nobody understood gorillas like Dian. (390, Hervorheb. im Original)

Breithaupt differenziert zwischen einem „weiter gestreute[n] Spektrum an Empathie-Effekten, die in der einen oder anderen Weise dazu führen, dass der Beobachter

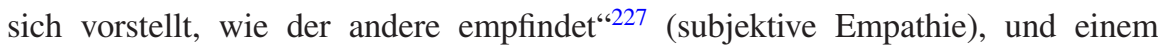
„vollständigen und korrekten Verstehen des anderen in seiner Situation“, welches „dabei mitdenken [würde], wie es ist, der andere zu sein“228 (akkurate Empathie),

\footnotetext{
${ }^{227}$ Breithaupt: Kulturen der Empathie, S. 22.

${ }^{228}$ Breithaupt: Kulturen der Empathie, S. 22.
} 
welches aber auch unmöglich sei. In Galdikas' Reflexion scheint Fossey und mit ihr die Ethologie der ,Trimates ‘ bemüht gewesen, diese nach Breithaupt unmögliche akkurate Empathie nicht nur strukturell zu ermöglichen, sondern auch methodisch zu systematisieren und zur Wissensgenerierung zu instrumentalisieren.

Galdikas thematisiert jedoch auch die mit der Frage der Empathie einhergehende Problematik der ,Objektivität“ in der Forschung. Sie geht daher zur Frage über: „Should you allow yourself to identify with your subjects, or should you remain uninvolved?" (391). Sie unterscheidet zwei mögliche Positionen für Feldforscher/innen: jene wenigen, die ,remain aloof and detached from their subjects“ (391) und die vielen, ,who study a single species or a single population for any length of time“ und die „,end up falling for their subject“ (291, Hervorheb. MS). Besonders Primatenforscher/innen neigen nach Galdikas zu Letzterem, zur affektiven Nahform für ihren Gegenstand, denn sie können der Menschenähnlichkeit der Tiere nicht widerstehen. Das Dilemma („Dian's dilemma“; „classic catch-22 situation“, 391), das daraus erwächst, ist für sie Folgendes: „If you don’t immerse yourself in your subjects' world, you only gather facts and figures, a computerized image; if you do become involved, you're accused of being unscientific" (391). Immersion und Involvierung sind Warnsignale für die Vertreter/innen einer engen Vorstellung wissenschaftlicher Objektivität; sie sind aber ironischerweise sowohl emotionale Bedingungen der Feldforschung, die dem ethnologisch formierten Muster der teilnehmenden Beobachtung folgt, als auch narratologische Elemente in der behavioralen Wissensproduktion.

Es geht Galdikas an dieser Stelle um das Problem der Doppelaufgabe von „research and conservation“ (392) der Forschung an „subjects who are endangered“ (392). Sie argumentiert hier jedoch auch programmatisch hinsichtlich einer Ethik, die als ,essence of being human“ die „,capacity for disinterested compassion“ (392, Hervorheb. im Original) veranschlagt, einer Art selbstloser Hilfeleistung, die nur praktizierbar ist, wenn die darin wirksame Empathie nicht in emotionale Überwältigung oder verallgemeinernde Projektion umschlägt. Äußerung dieser Ethik ist Galdikas' Naturschutz- und Auswilderungsprogramm, das eine ,liebevolle Erkenntnisweise“ verfolgt, wie sie Schilling als „Ergänzung zu einer objektivierenden Erkenntnis“229 vorschlägt, und damit zugleich den artspezifischen Bedürfnissen des Forschungsgegenstandes möglichst gerecht werden will. ${ }^{230}$

\footnotetext{
${ }^{229}$ Schilling: Liebe als Erkenntnisweise, S. 14.

${ }^{230} \mathrm{Im}$ Artenschutz-Spektrum gibt es hierzu mittlerweile auch Gegenentwürfe. Die Primatologin und Psychologin Anne Russon bezeichnet Galdikas' Camp Leakey als Programm der „,mother love“, dem seit 1991 das Orangutan Conservation Project (heute: Borneo Urangutan Survival Foundation) in Wanariset unter der Leitung des Forstwissenschaftlers Willie Smits gegenübersteht. In diesem wird der menschliche Kontakt für die Orang-Utans minimiert: „The love driving this program was tough love“. Habe Galdikas“ Auswil-derungsstation ,the look and feel of the bar scene in Star Wars“, gleiche Waraniset einem „orangutan Lord of the Flies“. Russon, Anne E.: Orangutans. Wizards of the Rain Forest. New York 2000, S. 100, 57, 131, Hervorheb. im Original. Siehe auch: https://orangutan.org/our-projects/research/camp-leakey (13.04.2018) und http://orangutan.or.id (13.04.2018).
} 


\subsection{Etüden der Kuschelprimatologie: Fiktion als Experimentalraum primatologischer Emotionalität}

\subsubsection{Rekapitulation: Erkenntnis qua Emotion}

Das dem Abschn. 2.2 vorangestellte Zitat aus dem Song Like a Virgin der Popsängerin Madonna aus dem Jahr 1984 mag auf den ersten Blick keinerlei Verbindung zur Primatologie und ihrem affektiven Potenzial aufweisen. Doch in diesen Zeilen wählen Madonnas Songwriter Billy Steinberg und Tom Kelly die Metaphorik der Wildnis und ihrer Bewältigung (,I made it through the wilderness/ Somehow I made it through"), sie sprechen von einem subjektiven Mangel („I was beat incomplete“), einem Verlorensein („Didn't know how lost I was“) als Wesenhaftigkeit, die ihrer Rettung und Vervollständigung entgegenblickt, von der Berührung als Signum eines neuen Zustands (,Touched for the very first time“), von einer Erkenntnis („Didn’t know how lost I was/ Until I found you“) und zugleich Erweckung, die durch das Fühlen entsteht (,,You made me feel“). ${ }^{231}$ Ausgerechnet ein erfolgreicher Song der frühen Madonna gemahnt so an die komplexe Beteiligung von Emotionalität an den Forschung- und Erkenntnisprozessen, aber auch an der Darstellung und Vermittlung der primatologischen Feldforschung, wie sie die Forschungsmemoiren schildern.

In den vorangegangenen Kapiteln ging es, ausgehend von dem bei Donna Haraway entliehenen ,Drama of Touch“ um die erste Periode der modernen Feldprimatologie und ihre affektiven Epistemologien. In dieser ersten Periode steht die Annäherung an das tierliche Andere im Mittelpunkt. Wie sich anhand der drei Kerntexte, Jane Goodalls In the Shadow of Man, Dian Fosseys Gorillas in the Mist und Biruté Galdikas' Reflections of Eden, und darüber hinaus nachweisen lässt, nimmt anders als in älteren Modellen der Affenbegegnung, die in ihrer Polarisierung von sentimentalem Selbst-Erleben und ,objektiver' Affektlosigkeit doch immer auf das Visuelle ausgerichtet waren, die Berührung zwischen Mensch und Tier als fühlbare Überwindung einer Spezies-Grenze dabei einen hohen Stellenwert ein. Sie ist nicht nur Indikator einer Veränderung in der Beziehung zwischen beobachtender Forscherin und beobachtetem Tier, sondern auch Teil einer Epistemologie, die Erkenntnis qua Emotion zulässt. Die taktile Begegnung ist zudem Medium der emotionalen Vermittlung einer Akzeptanz für den/die/das Andere/n in nächster Proximität und auch Manifestation einer Art sinnlichen Registrierens in der Forschungsbeziehung.

Wie sich zeigen ließ, ist ein Modell für eine solche empfindungsbasierte Erkenntnis, das sich für die Interpretation der diesbezüglichen Beschreibungen in den Forschungsmemoiren heranziehen lässt, bereits in der ästhetischen Reflexion zu finden: Anhand einer Feinanalyse des Gefühls des Erhabenen am Gorilla bei George Schaller, Dian Fossey und Robert Sapolsky konnte nachvollzogen wer-

\footnotetext{
${ }^{231}$ Alle Zitate Madonna: Like A Virgin.
} 
den, wie sich aus sinnesbasierter Empfindung affektive Erkenntnis schöpfen lässt, und zwar nicht als Novum der primatologischen Forschung sondern in der Tradition einer westlichen Ästhetik und ihres Wandels. Spätestens mit der Renaissance des Erhabenen als postmodernem philosophischen Gegenstand um 1990 wird das Gefühl des Erhabenen im Rückgriff auf Immanuel Kant in Verbindung gesetzt mit einer Thematisierung empfindungsbasierter Erkenntnis und ihrer emotionalen Komponenten. Gerade der Gorilla bietet sich in seinem natürlichen Lebensraum als Gegenstand für das Gefühl des Erhabenen und die daraus resultierenden Erkenntnismöglichkeiten - allen voran Möglichkeiten einer zugleich anthropologischen und zutiefst subjektiven Selbsterkenntnis - an. In Folge der popularisierten primatologischen Forschungsberichte und durchaus auch in Anknüpfung an das sentimentale Erleben des Jägers am Gorilla, wie es Du Chaillus Explorations and Adventures modellhaft zeigt, wird der Gorilla schließlich, wie Lukas Bärfuss' Roman Hundert Tage besonders deutlich werden lässt, zum kulturellen Topos des egozentrischen Erhabenen in der anthropozänen Postmoderne.

Wie die Darstellung in den Forschungsmemoiren darüber hinaus zeigt, ist es ganz grundsätzlich affektive Nähe, die als Bedingung für die Etablierung einer engen Forschungsbeziehung und als Instrumentarium empathischer Erkenntnisweisen dient. Mit Anleihen aus der Anerkennungstheorie ließ sich nachvollziehen, wie die Konstitution fruchtbarer Forschungsbeziehungen zwischen menschlichen und tierlichen Individuen von der Etablierung affektiver Nahformen abhängig ist, die individueller intersubjektiver Anerkennung und der normativen Integration in soziale Systeme dienen. Eine solche Anwendung annerkennungstheoretischer Überlegungen auf das in den Forschungsmemoiren Berichtete ist möglich, weil die Texte mittels narrativer Mittel (Figurenbenennung, individuelle Charakterisierungen, Erzählperspektiven, Mini-Narrative, Lebensgeschichten) Affen als textuelle Subjekte und Interaktionspartner/innen produzieren. Wissen über diese kann in der Situation der Feldforschung nur auf Basis gegenseitiger Anerkennung und besonders durch einfühlende Arbeitsweisen und narrative Empathie gewonnen werden.

Damit steht die methodische Privilegierung einer teilnehmenden und empathischen Beobachtung in der primatologischen Feldforschung in Verbindung mit affektiven Epistemologien, die vor oder jenseits des Geltungsanspruchs der Episteme der ,wissenschaftlichen Objektivität' in der jüngeren Moderne liegen: die Resomatisierung des Gefühls hin zum leiblichen epistemischen Prozess im ,Drama of Touch', der Adaption des aus der klassischen Ästhetik entstammenden Gefühls des Erhabenen, mit Strukturanleihen aus der sozialphilosophischen Anerkennungstheorie, und mit neuen Erkenntnissen zur epistemischen Rolle von Empathie und Narration.

\subsubsection{Inszenierung der Sentimentalität: Michael Apteds Gorillas in the Mist (1988)}

Gerade durch die anerkennungstheoretisch strukturierbare Beziehung zum tierlichen Individuum werden Erlebnisse mit den Affen im Rahmen der Feldforschungssituation 
auch zum Sozialkontakt. Dies begünstigt Assoziationen, die die Wahrnehmung der Autor/innen als Wissenschaftler/innen durch die Topoi von Sentimentalität und/ oder Zoophilie unterminieren. Der Interspezies-Kontakt scheint gerade durch das intime ,Kammerspiel' der Verhaltensforschung und seine heteronormativ ausgerichtete Besetzung überschattet von Bedenken und Insinuationen, die in die Texte hinein- und aus ihnen heraus in einen diskursiven Raum reichen, aus dem sich fiktionale Betrachtungen des Affe-Mensch-Verhältnisses bedienen. ${ }^{232}$ Gerade die Überschreitung der Tier-Mensch-Grenze hinsichtlich der Soziabilität macht die Primatologie für die Fiktion interessant. Nicht nur, weil das Forschungs- und Expeditionsnarrativ emotional ergiebig und ,thrilling ' ist, ${ }^{233}$ sondern gerade weil die Tier-Mensch-Grenze durch die primatologisch gestützten anthropologischen Erkenntnisse im 20. Jahrhundert - so ausgeprägt wie seit dem Skandal der Evolutionstheorie nicht mehr - nicht nur porös, sondern auch symptomatisch für die Bedürfnisse menschlicher Selbstbehauptung geworden ist. Literatur und Film greifen die Anregungen, die sich aus der Popularisierung der Primatologie durch die Forschungsmemoiren und die Vermarktung der Forschenden ergeben, auf und experimentieren mit den Tendenzen, die sich aus dem Weichen dieser Grenze ergeben. Beispielhaft kann diese These von der Fiktion als Experimentalraum für (auch grenzwertige) primatologische Emotionalität erneut am Gorilla untersucht werden. Es lässt sich auf Basis der Untersuchung des affeninduzierten Erhabenen am Gorilla argumentieren, dass Gorillas in zweifacher Weise Nutztiere für Menschen sind: einerseits als Forschungsgegenstand, der Aufschluss über die Evolution des Menschen und seiner Sozialformen liefern soll; andererseits als ästhetisches Objekt, an dem sich durchaus schwelgend die eigene Emotionalität im Gefühl des Erhabenen erleben lässt.

An dieser Stelle muss noch einmal an die ambivalent lesbaren Passagen aus Dian Fosseys Gorillas in the Mist erinnert werden, die sich mit den Gorilla-Männchen Digit und Peanuts befassen (vgl. Abschn. 2.2 und 2.4.2). Sie liefern ein gutes Beispiel für die Art vielschichtiger Emotionalität im Forschungskontext, welche den Raum für Spekulationen öffnet: Fosseys Wortwahl und Fokus auf die Individualität der beobachteten Tiere und ihre Belange lässt in den zitierten Passagen aus Gorillas in the Mist, wie bereits erwähnt, einen Subtext entstehen, der das Sinnliche semantisch verändert, und die Begegnungen als erotische interpretierbar macht. Der Blick des jungen Männchens Peanuts ist „unfathomable“ und hält Fossey ,[s]pellbound“ (141), bis sie seinen Blick erwidert und ein flirtiver ,exchange of glances“ (141) entsteht, welcher für Fossey zu einem ,unforgettable moment“

\footnotetext{
${ }^{232}$ Peter Høegs Roman spielt z. B. das King Kong-Motiv - weiße Frau, großer schwarzer Affe genussvoll in vollem Bewusstsein relevanter zeitgenössischer tierrechtlicher und ökologischer Diskurse aus und zeichnet dabei doch den Affen letztlich als edlen und weisen ,Wilden", der die weiße Frau aus ihrer selbstverschuldeten Unmündigkeit erweckt. Vgl. Høeg, Peter: Die Frau und der Affe. München 1997 (dän. 1996).

${ }^{233}$ Siehe bspw. die Thriller-Kombination des Motivs der Reise ins ,Innerste Afrikas“ mit der primatologischen Gebärdenforschung in Crichton, Michael: Congo. New York 1980.
} 
(141) wird. Sie ist freudig erregt und später wird dieser Gorilla in einer an Madonnas Song gemahnenden Formulierung zu ,the first gorilla ever to touch me“ (141). Nach der signifikanten Berührung - Hand in Hand - trommelt sich der Gorilla spannungslösend auf die Brust. Die Szene lässt sich so auch als Flirt mit dem Tier lesen, der die spröde Forscherin-Erzählerin Fossey mit einer interessanten erotischen Tendenz ausstattet. Diese setzt sich fort in der halben Stunde, die Fossey im Schoß des Gorillas Digit verbringt, den sie ausdrücklich (in Abgrenzung vom King-Kong-Mythos) als „my gentle Digit“ (199, Hervorheb. MS) charakterisiert. Die heimelige Szene im Regen muss erst durch so apologetische wie erklärende Einschübe gerechtfertigt werden (,a position that provided welcome warmth as well as an ideal vantage point from which to observe his four-year-old neck injury“, 199), um den Verdacht sentimentalen Genießens der Eigenaffektation und der zoophilen Grenzüberschreitung von sich zu schieben.

Die gleichnamige filmische Fiktionalisierung von Gorillas in the Mist $(1988)^{234}$ durch den Regisseur Michael Apted stellt Fosseys Forschungsalltag und den epistemologischen Mehrwert einer Affekte nutzenden Arbeitsweise denn auch im Sinne einer solchen Interpretation der Forschungsmemoiren zugunsten der Betonung von Emotionalität als Persönlichkeitsmerkmal zurück. Die affektive Beziehung zum Affen ist nicht länger Teil eines Forschungs- und Erkenntnisprozesses sondern Quelle eines auf den sentimentalen Genuss hin inszenierten emotionalen (Selbst-)Erlebens. Aus Fosseys autobiographischem Forschungsbericht wird dergestalt „The Story of Dian Fossey“ (so der Untertitel, TC 00:00:59). Der Film elaboriert dazu das emotionale Potenzial der bereits beschriebenen Szenen, indem er nicht nur mit Formen der Sentimentalität arbeitet, sondern ihnen auch einen deutlicheren erotischen Subtext verleiht. ${ }^{235}$

Als Dian Fossey wurde eine Schauspielerin besetzt, die zum Produktionszeitpunkt bereits durch eine besondere affektive Interspezies-Beziehung bekannt war: Sigourney Weaver, alias Ellen Ripley in Alien (1979) und Aliens (1986). ${ }^{236}$

\footnotetext{
${ }^{234}$ Apted, Michael: Gorillas in the Mist. Universal Pictures 1988. Timecode-Angaben im Folgenden im Text.

${ }^{235}$ Wie der Vorspann ausweist, basiert der Film auf ,the work of Dian Fossey“ und einem Artikel des Journalisten Harold T. P. Hayes (TC 00:02:27-30, Hayes, Harold T. P.: The Dark Romance of Dian Fossey: Caring For Gorillas More Than People was Fatal. In: Life vom November 1986, S. 64-71) und folgt dem Drehbuch von Anna Hamilton Phelan. Auf die problematischen Aspekte dieser Verfilmung zwischen kolonialistischem Blick und femininer Stereotypisierung wurde bereits ausführlich hingewiesen, vgl. Bingham, Dennis: Whose Lives Are They Anyway? The Biopic As Contemporary Film Genre. New Brunswick 2010, S. 295 ff.
}

${ }^{236}$ Scott, Ridley: Alien. 20th Century Fox 1979; Cameron, James: Aliens. 20th Century Fox 1986. Die Logik einer solchen Besetzungspolitik, die anthropologische Erkenntnis am außerirdischen und am primatischen Anderen parallelisiert, wird auch im Film gespiegelt: Michael Apteds Verfilmung beginnt mit folgender Setzung der Figur Leakey: „There are, I feel, only two major frontiers left: One is the exploration of space [...]; the other is the exploration of the past" (TC 00:00:37-00:01:14). So lässt sich durch Leakeys Empfinden - „I feel“ statt „I think“ - die primatologische Arbeit im Feld gerade am Beispiel der US-amerikanischen Forscherin Fossey nicht nur im Kontext eines anthropologischen Grenzdiskurses, sondern auch als Teil des affektiv auf- 
Die Figur Fossey ist hier stark auf das emotionale Erleben hin inszeniert: Der Film stellt Emotionalität als ihren prägenden Zug heraus und nimmt dabei einen Ausfallschritt in Richtung Sentimentalität vor. Bereits in der Eingangsszene wird Fossey durch Close-Ups ihres Gesichts in tiefer Faszination über eine Leakey-Vorlesung eingeführt. Auch der erste Sichtkontakt mit den Freiland-Gorillas wird über die Nahaufnahme des Gesichts als Leinwand emotionaler Ausdrucksformen bewertet. Das affektive Erleben wird hier dezidiert als nicht sprachlich nachformbar, aber als Wert an sich präsentiert: „There aren't words to describe my emotions“ (TC 00:38:37-40), sagt Weavers Fossey z. B. im Voice-Over einer Collage von Fossey-Zitaten und Leakey-Briefauszügen, um die Bedeutung der Nähe zu den Gorillas hervorzuheben. Emotionalität ist hier jedoch nicht Teil einer Welterfassung durch Sinne, Empfindungen und Gefühle, und schon gar nicht Teil eines Erkenntnisprozesses. Sie dient vielmehr einerseits der Einbettung Fosseys in ein heterosexuelles Narrativ romantischer Liebe - der Film liest ihr Verhältnis zu den Forschungstieren kontinuierlich als Romanze und betont eine menschliche Affäre - und andererseits der Abwertung ihrer Forschungsarbeit durch ein inhärent negatives Verständnis von Emotionalität und die Inszenierung von sentimentalem Genießen. ${ }^{237}$ Wie Dennis Bingham bereits thematisiert hat, werden Fosseys Handlungen im Film stetig einem dichotomen Verständnis von Affekt und Intellekt folgend als ,consistently wrong, driven by emotion rather than by vision or goals“238 dargestellt. Anders als in Dian Fosseys Text gibt es hier gezielt gesetzte Hinweise, die das Narrativ der romantischen Forschungsbeziehung weiter in Richtung Speziesdiffusion treiben. So berichtet die Film-Fossey im Voice-Over, der Silberrücken der ersten beobachteten Gruppe lasse keinen Blick von ihr (,,never takes his eyes of me“, TC 00:38:19-22) und die Gorillas seien.

quite confused as to my species. I've gotten them accustomed to me by mimicking them, and they are fascinated by my facial grimaces and other actions that I wouldn't be caught dead doing in front of anyone. I feel like a complete fool, but this technique seems to be working (TC 00:38:57-00:39:15). ${ }^{239}$

geladenen, nordamerikanische Identität stiftenden Frontier-Diskurses lesen. Zum Mythos ,Frontier' vgl. Slotkin, Richard: Regeneration Through Violence: The Mythology of the American Frontier: 1600-1860. Norman 2000; Slotkin, Richard: Gunfighter Nation: The Myth of the Frontier in Twentieth-Century America. Norman 1998.

${ }^{237} \mathrm{Zu}$ den Implikationen der Kategorie des Sentimentalen vgl. Dorschel, Andreas: Sentimentalität. Über eine Kategorie ästhetischer und moralischer Abwertung. In: Perspektiven der Philosophie 31 (2005), S. 11-22. Statt des Gefühls des Erhabenen setzt der Film einen Akzent auf den affektiven Genuss qua religiöser Verzückung im mimischen Repertoire der Figuren. Konsequenterweise heißt es angesicht der vernebelten Berggipfel und grasenden Gorillas hier: „This is as close to God as you get“ (TC 01:37:21-25).

${ }^{238}$ Bingham: Whose Lives Are They Anyway? S. 302.

${ }^{239}$ Während der Film chronologisch Fosseys Gorillas in the Mist folgt, stützt er sich in der Textwiedergabe auf Fosseys private Aufzeichnungen und ihre Briefkorrespondenz. In Fosseys Gorillas in the Mist heißt es vergleichsweise zum Thema der Habituation durch ,belch vocalizations", denen Fossey besondere wissenschaftliche Aufmerksamkeit widmet: „The sound serves as the 
Männlichen Gorillas gegenüber zeigt sie sich in der Pose kauernder Unterwürfigkeit (explizit rät sie im Film: ,act submissive“, TC 01:00:24-27), bis Fossey mit dem körperlich-mimischen Repertoire der Verliebtheit auf die Akzeptanz des Gorilla-Männchens reagiert: starrer Blick, weiche Gesichtszüge, geweitete Augen, bebende Lippen. Die ,First Touch“-Szene, hier mit Digit statt Peanuts durchgeführt, dient vor allem dazu, jenes romantische Narrativ zu bestätigen. Dies geschieht durch die Einführung einer dritten Figur in die Szene: Bob Campbell, der im Dienste der National Geographic Society Fossey und die Gorillas filmen soll. ${ }^{240}$ Die Erstbegegnung mit Campbell verläuft analog zu jenen mit den Gorilla-Männchen. Auch hier starrt Weavers Fossey fasziniert auf das männliche Individuum, neigt den Kopf leicht und bleibt sprachlos. Im Film ist es Campell, der Fossey auffordert, in die Nähe Digits (hier anders als im Text ein Silberrücken) zu gehen: „Could you go over to him?“" „Digit?“; Nicken; „Now?“; „Is it alright?“ (TC 01:00:56-01:01:07). Es geht hier nicht um die Markierung einer Anerkennungsbeziehung durch ein gestisches Zeichen, filmisch dokumentiert, sondern um die Inszenierung und Lesart der außergewöhnlichen Tier-Mensch-Beziehung für die Kamera. Fossey biegt sogar einen Zweig zur Seite, damit Campbells (und damit unser) Kamerablick freie Sicht auf die Protagonist/innen der Szene, das Gespann Digit-Fossey, hat (TC 01:01:54-55). Nun vollzieht sich die Szene anders, als dies von Fosseys Text her bekannt ist: Digit imitiert Fossey zunächst darin, wie sie Gorilla-Verhalten nachahmt. Fossey lehnt sich daraufhin mit Blick auf Digit zurück und spielt mit den Fingern an ihrem Gesicht entlang. Digit scheint sie anzublicken und berührt ihre Hand. Weavers Fossey schaut verzückt und streichelt im Close-Up auf den Händedruck die Gorilla-Pranke. Der Moment wird dadurch betont, dass die Kamera in Nahaufnahme in Campbells Gesicht hineinzoomt und es in erstarrter Faszination zeigt. Digit steht auf, trommelt sich auf die Brust, Fossey springt kurz hoch, lehnt sich dann aber wie post-orgasmisch zurück und spürt mit den Fingern dem Händedruck nach (TC 01:03:35-54). Wieder wird dies mit einem Schnitt auf Campbells angespanntes Gesicht kontrastiert. Die Anspannung von Szene und

perfect communication for humans to imitate when initiating contacts with gorilla groups either partially or totally obscured in vegetation. By its use I can inform the animals of my presence and allay any apprehensions they might have on hearing the noise of vegetation broken near them. It is an extraordinary feeling to be able to sit in the middle of a resting group of gorillas and contribute to a contented chorus of belch vocalizers" ( $G M, \mathrm{~S} .53$ f.).

${ }^{240}$ Vorab ist in fast jeder Einstellung der ruandische Feldassistent Sembegare in der Rolle des loyalen einheimischen ,Boys` zu sehen. Dieser ist jedoch weder als männlicher Gegenpart noch als Partner des emotionalen Austausches von Bedeutung, wie im Anschluss an die Bemerkung, es gäbe keine Worte, ihre Emotionen zu beschreiben, deutlich wird: „My only regret is that apart from Sembegare I don't have anyone to share this with“ (TC 00:38:48-52, Hervorheb. MS). Folglich bedarf es einer validen männlichen menschlichen und weißen Figur, um das romantische Narrativ zu stützen. Auf die rassistischen und kolonialen Unter- und Obertöne sowohl von Fosseys Text als auch der ihr darin folgenden Verfilmung hat bereits Bingham verwiesen; vgl. Bingham: Whose Lives Are They Anyway?, S. $296 \mathrm{ff}$. 
Gesicht wird in der nächsten Einstellung entladen: Campbell und Fossey lassen zu Musik über Fotografien in der Hütte den Tag ausklingen - Flirterei, Artikulationsschwierigkeiten, Kuss. Im Anschluss wird im Bett Whiskey getrunken und Campbell kommt auf den äffischen Konkurrenten zurück: „I was amazed when he came so close to you“. Fossey erwidert betont mehrdeutig: „Digit and I have a strange connection“" (TC 01:05:46-53; Hervorheb. im Original).

Von hier an steht zwar zunächst die menschliche Romanze im Vordergrund. Der Unterton zoophiler Grenzüberschreitung klingt jedoch in der affektiven Figurenzeichnung nach, mit welcher der Plot vorangetrieben wird. Das Scheitern der Affäre mit Campbell und der Verlust von immer mehr Forschungstieren lassen Fossey in eine Spirale erratischen Verhaltens taumeln, in der Rachegefühle aufgrund der Tötung der Gorillas durch Wilderer vorherrschen. Auch das unausweichliche Ende jener Spirale und des Films lässt sich im Sinne einer Romantisierung des Forschungsverhältnisses bei gleichzeitiger Irrationalisierung der Forscherinnenfigur lesen: Weavers Fossey, körperlich gezeichnet von Lebensstil und Forscherinnenhabitus, betrachtet auf dem Bett sitzend mit verliebtem Blick ein Bild von Digits Nachwuchs - hier anders als in Fosseys Text ein Männchen. ${ }^{241}$ Wie um die affektive Nahform als heterosexuelle Liebe zu präzisieren, singt im Hintergrund vom Tonband Peggy Lee den Liebessong Sugar (That Sugar Baby o' Mine $)^{242}$. Fossey spricht die Fotografie mit einem ästhetischen Urteil an, zweifach, indem sie vom noch gegenständlich-tierlich inklinierten ,That's a beauty“ (TC 02:00:54-55) zum subjektivierend-menschlichen „You are beautiful“ wechselt (TC 02:01:26-28). Sie lehnt sich zurück, küsst die Fotografie sogar, bevor sie, das Bild vor der Brust, einschläft.

Wie um dieser Grenzüberschreitung in Form tier-menschlicher affektiver Nahformen symbolisch Einhalt zu gebieten, folgt der Szene die Ermordung Fosseys nur sichtbar durch einen Schatten an der Wand. Emblematisch tropft schließlich Blut auf die Schwarz-Weiß-Fotografie von Digits Nachwuchs. Die endgültige Einschreibung des Verhältnisses von Fossey zu ihrem Forschungsgegenstand als romantische Liebe vollzieht sich in der Szene der Beisetzung Fosseys neben Digits Grab in Karisoke. Ihr getreuer Sembegare verbindet - der Brauch wurde vorab als ,indigene Kultur' eingeführt (,The circle joins two souls and makes them one forever", TC 00:42:18-22) - die beiden Gräber mit Steinen wie die zweier Liebender, während der Abspann läuft (TC 02:05:02-02:06:47).

\footnotetext{
${ }^{241} \mathrm{Vgl}$. GM, S. 211: „Digit's perpetuation was an extraordinarily beautiful female infant with long fluttering eyelashes that framed bright sparkling eyes“. Im Film dagegen ,erkennt“ („I know you.", TC 01:57:11-13, Hervorheb. im Original) Fossey das Gorilla-Kleinkind an zwei zusammengewachsenen Fingern (,fourth and fifth digits webbed“, TC 01:57:09-11), und das Geschlecht wird sogleich durch das signifikante Brusttrommeln ausgewiesen.

${ }^{242}$ Lee, Peggy/Matty Matlock's Dixielanders: Sugar (That Sugar Baby o‘ Mine). Text und Melodie v. Maceo Pinkard/Edna Alexander/Sidney D. Mitchell. Aus dem Film Peter Kelly's Blues (Regie: Jack Webb, Warner Bros. 1955).
} 


\subsubsection{Experimentelle Zoophilie: Peter Goldsworthys Wish (1995)}

Bleibt Apteds Gorillas in the Mist-Adaption bei aller Insinuation noch ambig, gestaltet Peter Goldsworthy die affektive Nähe zwischen Mensch und Gorilla als romantische Beziehung in seinem Roman Wish explizit und körperlich und fügt sie in zeitgenössische Tierrechts- und Forschungsdebatten ein. ${ }^{243}$ Goldsworthy nutzt dabei die Möglichkeiten von Fiktion als Gedankenexperiment, um über die allein logischen Konsequenzen wissenschaftlicher Entwicklung hinauszugehen und die affektive Resonanz der primatologischen Forschungspraktiken und -ergebnisse zu erschließen.

Thomas Macho und Annette Wunschel haben zwar davor gewarnt, den Begriff des Gedankenexperiments formal und historisch auszuweiten, denn sie sehen das Gedankenexperiment von der Fiktion im Allgemeinen durch seinen punktuellen und strategischen Einsatz geschieden. Dabei rekurrieren sie aber wesentlich auf eine Definition der kontrafaktischen Science Fiction, die für das Gedankenexperiment ebenfalls eine Veränderung der Welt „,nur an einer einzigen Stelle“, ${ }^{244}$ aber die Überprüfung dieser Veränderung ,,an vielen anderen Stellen“245 annimmt. Dies trifft auf Goldsworthys Roman zu, der in einer Welt spielt, in der eine genetische Mensch-Gorilla-Hybridisierung möglich ist, die aber ansonsten unserer gleicht. Darüber hinaus sieht Sigrid Weigel Literatur als potenzielles Feld von Gedankenexperimenten ,zu der nicht unerheblichen Frage, was aus neuen wissenschaftlichen Erkenntnissen wird, wenn sie ihren Sitz im Leben der Subjekte einnehmen“. ${ }^{246}$ Nicht nur ist dies - das Übersetzen neuer wissenschaftlicher Erkenntnisse in das Leben eines literarischen Subjekts - hier der Fall. Goldsworthy betrachtet den literarischen Blick auf Wissenschaft selbst als Gedankenexperiment: „What I liked about science-fiction was its feedom of inquiry. Its

\footnotetext{
${ }^{243}$ Dass Goldsworthys Roman auf fundierter Recherche beruht, hat Griem bereits nachgewiesen. Siehe Griem: Monkey Business, S. 278 ff. Diese Recherche wird auch intratextuell durch die Nennung etlicher einflussreicher Publikationen zum Thema und paratextuell durch einen betreffenden Anhang, der explizit auf das Great Ape Project verweist, ausgestellt; vgl. Goldsworthy, Peter: Wish [1995]. Melbourne 2013, S. 383. Fortan, wenn zur Unterscheidung nötig, als WI geführt; Seitenangaben im Text. Mittels seines Figurenpersonals reflektiert Goldsworthy zudem unterschiedliche Positionen der angesprochenen Debatten: der intellektuelle Tierrechts-Theoretiker und Philosoph Clive, die mütterliche Tierliebhaberin und Tierärztin Stella, der ambivalente Forscher bzw. Experimentator Terry, und der Protagonist und Ich-Erzähler J.J., der stellvertretend für die Leserschaft zunächst naiv auf das Thema Tier-Mensch-Beziehung stößt und erst an die Problematik herangeführt werden muss.

${ }^{244}$ Wunschel, Annette/Macho, Thomas: Mentale Versuchsanordnungen. In: Dies. (Hg.): Science \& Fiction. Über Gedankenexperimente in Wissenschaft, Philosophie und Literatur. Frankfurt a. M. 2004, S. 9-14, hier S. 9.

${ }^{245}$ Wunschel/Macho: Mentale Versuchsanordnungen, S. 9.

${ }^{246}$ Weigel: Das Gedankenexperiment, S. 201.
} 
stories were a species of what philosophers now like to call thought experiments. [...] [S]tories could do more than describe, or explain, or define, or hypothesize. Stories might also possess (or be possessed by) certain magical properties, musical and emotional properties“". ${ }^{247}$

Wish spielt vordergründig nicht im primatologischen Forschungsalltag, verhandelt jedoch primatologische Forschungsfragen und das Verhältnis von Mensch und Menschenaffe: Der Protagonist J.J. wird durch die Anstellung als Gebärdensprachlehrer für das Gorilla-Weibchen Eliza/Wish nach und nach nicht nur an verschiedene primatologische und anthropologische Fragestellungen und Themen herangeführt, sondern kann aus der Ich-Perspektive die emotionale Interaktion mit dem Affen-Gegenüber erproben. J.J. nimmt ein sexuelles Verhältnis zu Wish auf, dessen Entdeckung durch andere Figuren des Romans schließlich nicht nur $\mathrm{zu}$ einem Strafprozess wegen, bestiality ${ }^{248}$ gegen J.J. und zu öffentlicher Aufmerksamkeit für die Personenrechtsdebatte, sondern auch dazu führt, dass Wish in einen Zoo verbracht wird, in dem sie sich suizidiert.

Auch in diesem fiktionalen Text spielt Emotionalität eine Hauptrolle. Diese bewegt sich auf der Bühne der bekannten Dichotomisierung von Rationalität und Emotionalität, wobei hier jedoch die Sympathieträger/innen dem Gefühl zusprechen, während negative Charakterisierungen anhand von fehlender Emotionalität vorgenommen werden. Durch die Figurenkonstellation wird sowohl (im Paar Clive und Stella) männlicher Intellekt von weiblichem Affekt als auch zwischen zwei verschiedenen Frauentypen differenziert: Die Frauenfiguren als Vertreterinnen akademischer Rationalität (Jill, Linda) sind hier durchweg als ,unterkühlt' und unemotional negativ gezeichnet und dem Protagonisten hinderlich, während zunächst Stella und dann vor allem Wish als emotional aktive und wohlgefällige, ,warmherzige" weibliche, aber eher irrationale Figuren inszeniert werden. J.J. selbst wird nicht nur durch die autodiegetische, intern fokalisierte Erzählweise, sondern auch als Vertreter der australischen Gebärdensprache Auslan ausdrücklich als fühlendes Individuum in Szene gesetzt. ${ }^{249}$ Emotionalität besitzt hier einen vorgängigen epistemischen Wert; sie bietet ein Erkenntnisinstrumentarium, das intuitiv zugänglich ist und nicht erst erlernt werden muss - und daher Mensch und Tier verbinden kann: Am deutlichsten wird diese Auffassung, welche Emotionen in einer solchen Dichotomie von Vernunft und

\footnotetext{
${ }^{247}$ Goldsworthy, Peter: Honk If You Love Science. In: Island Magazine 54 (Autumn 1993), S. 40-43, hier S. 40.

${ }^{248}$ Das Deutsche kennt keinen vergleichbaren juristischen Begriff für den sexuellen Verkehr mit Tieren, sehr wohl aber den Tatbestand, der bspw. nach TierSchG § 3 Satz 1 Nr. 13 (Deutschland) und TSchV Abschn. 2, Abschn. 3, Art. 16j (Schweiz) verboten und geahndet wird.

${ }^{249}$ Die Gebärdensprache wird hier hypothetisch charakterisiert als der Emotionalität stärker verbunden als Schrift- und Lautsprache: „And here, as I scribble these words, I touch the heart of my problem: how, above all, to translate feelings, so easily and naturally expressed in the dance of Sign, so much a part of the actual vocabulary of Sign, into words?" (WI, S. 7, Hervorheb. im Original). Gebärdensprache sei „more natural to us“ (S. 16) und agiere wie ein Lebewesen: „Sign is lifeless the moment it hits the page [...]. Sign moves and breathes, whispers, shouts,
} 
Gefühl, Mensch und Tier verwurzelt, wenn J.J. versucht, der empörten Vegetarierin Wish zu erklären, weshalb er Fisch gegessen habe. Hier heißt es: „I tried to appeal to her emotions instead [anstelle der ,learnt logic '], to the part of her, that part of all of us, which is more purely animal, and pre-rational“" (255).

Die der Tier-Mensch-Beziehung inhärente affektive Nähe wird in diesem Roman nicht nur exploriert, sondern auch hinsichtlich ihrer ethisch-rechtlichen Relevanz betrachtet: Lässt sich der rechtliche Tier-Status eines experimentell und habituell modifizierten Gorilla-Weibchens, das nur unter Menschen aufgewachsen ist und in menschlichen Zeichensystemen kommunizieren kann, aufrechterhalten? Und nimmt man die Forderung nach Personenrechten für Menschenaffen ernst, muss man sich dann nicht auch mit dem Recht auf eine selbstbestimmte Sexualität auseinandersetzen? Wish erzählt dabei jedoch eine weitaus problematischere Geschichte: Die Beziehung zwischen einem sich in der adoleszenten Pose aufbrausender Emotionalität gefallenden Menschen-Mann in der Lebenskrise und einer kindlichen Gorilla-Jugendlichen, die sich in einem pädagogischen Verhältnis befinden. ${ }^{250}$

Dass der Roman der Vorstellung von einem ,pädagogischen Eros ‘ anhängt, zeigt sich bereits auf den ersten Seiten, wenn der Protagonist und Erzähler J.J. erläutert: „The best teacher-student bonds are a kind of love, I think, selfless and pure. And more equal than at first they seem - dependent on a likeness of mind, a journeying together" (11). ${ }^{251}$ Der Lehrer und seine Schülerin, der Mensch und die Gorilla, treten in ein Beobachtungsverhältnis, das einer erotischen Dramaturgie folgt: Wishs Blick hängt an J.J.s Händen, seiner jedoch an ihrem Körper: „If Wish spent those first few weeks studying my hands, I spent them studying her. Her hands, her dark eyes, the glossy pelt that covered much of her body. The black-crepe skin of her

pirouettes, jives..." (S. 23). Die Vermittelbarkeit von Gebärdensprache an Lautsprachliche wird in Wish mehrfach im Vokabular des Tanzes und der Lyrik thematisiert, jenen vermeintlich affektnahen Künsten. Vgl. obig ,the dance of Sign“ und „pirouettes, jives“, sowie Wishs „Sign poem“ ,Crowds of Wishes“, „[a]n expression of great beauty“ (S. 156), auf das J.J. eine körperliche (affektive) Reaktion zeigt: „I shivered suddenly, involuntarily; [...] the poetry of these shapes was exhilarating, magical. Goose bumps pricked my neck and back" (S. 156).

${ }^{250}$ Diese Charatkeristika stellt der Text deutlich heraus: J.J. lebt nach dem Ende seiner Ehe, für das er wiederholt die akademischen Ambitionen seiner Ex-Frau verantwortlich macht, wieder bei seinen Eltern und verfällt in das Rollenmuster eines Teenagers, der finanziell und emotional von diesen abhängig ist. Das affektive Repertoire jugendlicher Aufständigkeit und elterlicher Repression zeigt sich ebenso wie die schnelle Affizierbarkeit durch neue Ideen, so angesichts von Clives Manifest für den Vegetarismus. Über Wishs Alter trifft der Text unterschiedliche Aussagen: Ihr kindliches Aussehen (,She was almost adult sized, but her face was surely not the face of an adult gorilla. Her head [...] - a child's big head“, S. 124; ,an overgrown child-ape“, S. 170), das biologische Alter, die kognitiven Fähigkeiten, ihr Zimmer (,The room might have belonged to any human child“, ebd., 197) und das geschlechtsreife Verhalten indizieren unterschiedliche Alterseinschätzungen. Das 8-jährige Gorilla-Weibchen scheint jedoch eher einer jungen Pubertierenden im Alter von J.J.s 11-jähriger Tochter zu gleichen, als der Halbwüchsigen, als die sie schließlich bezeichnet wird (,an adolescent - a teenager, in human years“, S. 293).

${ }^{251}$ Vgl. zur Kontinuität dieses Themas im Werk Goldsworthys: Bradley, James: Animal Form. In: Peter Goldsworty: Wish. Melbourne 2013, S. VII-XIII, hier S. IX. 
bare breasts and armpits. Her small, fine elf ears“ (206). Bald dringt J.J. - noch im Lehrverhältnis - in diesen beeindruckenden Körper ein, wenn er im Versuch, ihr Flüstersprache beizubringen, mit seinen Fingern in Wishs Mund die Laute formt (,I spent some time with my fingers inside her mouth“, 224), und er schiebt ihre in seinen (,I guided her thumbish fingers into my mouth, to feel my tongue and teeth positions“, 224). Goldsworthy schreckt auch nicht vor einer Gitarren-Szene zurück, in der J.J. ,sat behind her, one hand reaching round her broad back to grasp her strumming hand, the other guiding her fingers into a basic G-chord" (227). Bald weist ihn Stella auf „Wish's growing puppy love, adoring even when she was teasing me“ hin, doch diese Wahrnehmung ,added immensely to the pleasure of those days" (230).

Der Interspezies-Sexualakt ist hier Peripetie nicht nur der Dramaturgie eines intellektuellen Diskurses, der Personenrechte für Menschenaffen fordert, sondern auch der den Roman sättigenden erotischen Emotionalität als Medium der Erkenntnis und Selbsterkenntnis: J.J. bezeichnet ihn als „central to this story what follows is the story, in essence“" (303, Hervorheb. im Original). Die wachsende Zuneigung zwischen J.J. und Wish wird in jenem Moment als „love“ identifiziert, als Wish sich J.J. ,präsentiert' wie es im primatologischen Vokabular heißt: „She was presenting to me, a word that doesn't exist in any dictionary in Sign“ (265). J.J. flieht, sieht sich jedoch gezwungen, die Form seiner affektiven Beziehung zu Wish vor sich selbst zu identifizieren: „A tutor's love for a gifted pupil? A foster-parent's love? These certainly. These at the least“ (273); „I realised that I loved more than merely, being with' her. I loved her. [...] I knew also, equally clearly, that those feelings were returned. Wish loved me, I loved her" (273, Hervorheb. im Original). Wie deutlich anhand körperlicher Symptomatik beschrieben, ist dies eine Selbst-Wahrnehmung durch die emotionale Verhandlung: Während Stellas fordernde, menschliche sexuelle Avancen J.J. soweit verstören, dass er seine geschlechtliche Identität bedroht sieht (,My cock retracted completely, on the verge of becoming a vagina“, 263), wird er durch die äffische weibliche Aufforderung zur Begattung virilisiert (,My hard cock still refused to sag; I felt harder than I had felt for many years“, 266).

Die Umkehrung des King-Kong-Narrativs („Higher on that first wall was a sketch of the same tubby figure - mine - swinging through trees, flying between branches, airborne, with wings of crayon sprouted from my back; and Wish herself waiting, hands outstretched, to catch me“, 198 f.) scheint zunächst einen von kulturellen Stereotypen unverstellten Blick auf das Thema des Interspezies-Sex zu ermöglichen. Doch andererseits impliziert die latente Misogynie des Protagonisten und Erzählers, zumindest gebildeten Frauen gegenüber, an gerade diesem Geschlechterverhältnis wiederum ein Weiblichkeitsbild, das durch Kindlichkeit und ,natürliche" Animalität lockt und den betreffenden Mann aus der Position des späten und trotzigen Teenagers in jene des mächtigen Silberrückens versetzt: „I felt, for once in my life, beautiful: a giant of a man, a human silverback, in full sexual rut" (306). Dadurch ist schließlich der Interspezies-Sex auch kein Akt der selbstbestimmten Sexualität, der Wish zu einem rechtlichen Subjekt machen würde. Wish scheint in der menschlichen Perspektive des Textes schlicht ihren 
,natürlichen' Instinkten und Bedürfnissen zu folgen, wenn nicht sogar eine Variation des (bereits in Wishs ,Geburtsnamen“ Eliza angedeuteten) Pygmalion-Narrativs zu liefern. Der Verkehr dient J.J. zur Wiedererweckung seiner Sexualität und Selbsterkenntnis - in Madonnas Worten: „like a virgin/ touched for the very first time“. Julika Griem liest denn auch den Sexualakt zwischen J.J. und Wish - unter leichter Veränderung der Erzählchronologie - als letzten Akt einer Selbstentfremdung der Gorilla, ,die der Selbstfindung des menschlichen Protagonisten und Erzählers kritisch gegenübergestellt wird““. ${ }^{252}$ Es ist wohl eher Wishs Selbsttötung durch den Strick im Zoo, der als Akt des selbstbestimmten Handelns und nicht als Verhalten oder Instrumentalisierung fungiert. Während der Sexualakt die Sensationslust der Öffentlichkeit befriedigt und entsprechend öffentlich besprochen werden kann, muss der letzte Akt des selbstbestimmten freien Handelns, die Selbsttötung, konsequenterweise als Unfall vertuscht werden: „LOVE APE DIES IN FREAK ACCIDENT“ (370) lautet die Zeitungsüberschrift zu einem emblematischen Bild: „Wish, clearly dead, hung from one of the ropes in her zoo enclosure. Definition was poor, but some sort of noose seemed to have been knotted around her neck" (371).

Anders als Julika Griem dies ansetzt, lässt sich m. E. der Roman nicht als Versuch lesen, eine nicht-menschliche Perspektive, d. h. „den Betrachterstandpunkt, die Erfahrungen und Empfindungen eines tierischen Subjektes" ${ }^{\prime 253}$ zu simulieren. Als solcher würde der Versuch schlicht daran scheitern, dass alles, was wir über Wish erfahren, durch J.J.s autodiegetische Perspektive interpretiert ist. Und J.J. ist selbst im Bemühen, Wish als Subjekt anzuerkennen, ein zu selbstbezogener Erzähler, als dass er uns Aufschluss über Wishs Subjektivität geben könnte. So reagiert J.J. auf die Selbsttötung Wishs auch signifikanterweise mit einer Selbstbestätigung: ,,Yes, ' I called after him, ,I loved her.' Not so much to gratify him, as to tell someone. Anyone“ (371, Hervorheb. im Original). Was der Roman somit thematisiert, indem er das emotionale (Selbst-)Erleben seines menschlichen Protagonisten elaboriert, ist die Problematik eines Zugangs zu genuin nicht-menschlichen Perspektiven, der nur über menschliche Ausdrucks-, Zeichen-, wenn nicht sogar Wahrnehmungs- und Beschreibungssysteme entstehen kann, somit also bereits immer eine Übersetzungsarbeit beinhaltet. ${ }^{254}$

\footnotetext{
${ }^{252} \mathrm{Vgl}$. Griem: Monkey Business, S. 286.

${ }^{253}$ Griem: Monkey Business, S. 278.

${ }^{254}$ Vgl. auch Griem: Monkey Business, S. 283 ff. In einem weiteren Roman, der wesentlich konsequenter die äffische Perspektive ergreift, Hales The Evolution of Bruno Littlemore, muss dies bezeichnenderweise unter dem Vorzeichen eben solch einer ,Evolution' des schimpansischen Erzählers und Protagonisten zum Menschen geschehen, sodass das Schimpansische der Erzählperspektive zunehmend in den Hintergrund gedrängt wird. Siehe Hale, Benjamin: The Evolution of Bruno Littlemore. New York 2011. Vgl. auch Richter: Ape Meets Primatologist.
} 
In beiden fiktionalen Texten, dem Film ebenso wie dem Roman, wird Emotionalität als Komponente und Implikation von Mensch-Tier-Verhältnissen und primatologischer Forschung auch hinsichtlich ihrer Konsequenzen exploriert. Der Film Gorillas in the Mist nutzt dies, um im Rahmen des Biopics Dian Fosseys emotionales Verhältnis zu ihrer Forschung und ihren Forschungstieren nicht allein als Leidenschaft, sondern als Romanze zu zeichnen. Emotionalität wird dabei nicht als Teil eines Forschungs- und Erkenntnisprozesses betrachtet, sondern als Persönlichkeitsmerkmal gedeutet und als Sentimentalität im Sinne eines „absichtliche[n] Schwelgen[s] in der Empfindung“"255 dargestellt, an dem auch die Zuschauenden teilhaben können. Wish dagegen elaboriert Emotionalität aufwertend als Bestandteil von Lehr- und Lernverhältnissen, und somit trotz eines Emotionsverständnisses, das Emotionen als nicht-rational betrachtet, als Erkenntnismittel. Vor allem aber nimmt Emotionalität die Funktion eines primordialen Zugangs zum Tier ein. Emotionalität ist hier - ähnlich wie bei Goodall - ein Medium der Kommunikation, das nicht erlernt werden muss, sondern speziesübergreifend geteilt wird.

Während der Film Gorillas in the Mist den Vorwurf der ,Kuschelprimatologie“ illustriert, indem er die taktile und emotionale Selbsterfahrung am Tier in Bild und Ton genussvoll inszeniert, wird die emotionale Nähe zwischen Mensch und Gorilla bei Goldsworthy auf ihre Folgen hin gedacht: thesenhaft und diskursiv für den weiblichen Gorilla als Gegenstand rechtsphilosophischer Diskussionen; vor allem für den menschlichen Mann als krisenhafte Selbstfindung in speziesübergreifender Emotionalität - „Didn't know how lost I was/ Until I found you [....]/ But you made me feel“. Als populäre Laboratorien für die Gestaltung und die Implikationen von Emotionalität im interprimatischen Kontakt machen es diese fiktionalen Texte der wissenschaftlichen Primatologie nicht unbedingt leichter, Emotionalität zu erfahren, systematisch zu dokumentieren und transparent abzubilden, um sie als Teil von Erkenntnisprozessen zu nutzen, wie sich an der Entwicklung der Primatologie und ihrer Leitideen zeigt. Umso mehr Emotionalität jedoch aus den Forschungsberichten und Ergebnisdiskussionen ausscheidet, desto wichtiger werden fiktionale und populärwissenschaftliche Texte als Medien analytischer und spekulativer Exploration.

\footnotetext{
${ }^{255}$ So zitiert Dorschel aus Friedrich Theodor Vischers Ästhetik, vgl. Dorschel: Sentimentalität, S. 6; Vischer, Friedrich Theodor: Aesthetik oder Wissenschaft des Schönen. Zum Gebrauche für Vorlesungen. Bd. 2,2: Die Lehre von der Phantasie. Hg. von R. Vischer. Reutlingen/Leipzig 1848, S. 513.
} 
Open Access Dieses Kapitel wird unter der Creative Commons Namensnennung 4.0 International Lizenz (http://creativecommons.org/licenses/by/4.0/deed.de) veröffentlicht, welche die Nutzung, Vervielfältigung, Bearbeitung, Verbreitung und Wiedergabe in jeglichem Medium und Format erlaubt, sofern Sie den/die ursprünglichen Autor(en) und die Quelle ordnungsgemäß nennen, einen Link zur Creative Commons Lizenz beifügen und angeben, ob Änderungen vorgenommen wurden.

Die in diesem Kapitel enthaltenen Bilder und sonstiges Drittmaterial unterliegen ebenfalls der genannten Creative Commons Lizenz, sofern sich aus der Abbildungslegende nichts anderes ergibt. Sofern das betreffende Material nicht unter der genannten Creative Commons Lizenz steht und die betreffende Handlung nicht nach gesetzlichen Vorschriften erlaubt ist, ist für die oben aufgeführten Weiterverwendungen des Materials die Einwilligung des jeweiligen Rechteinhabers einzuholen. 